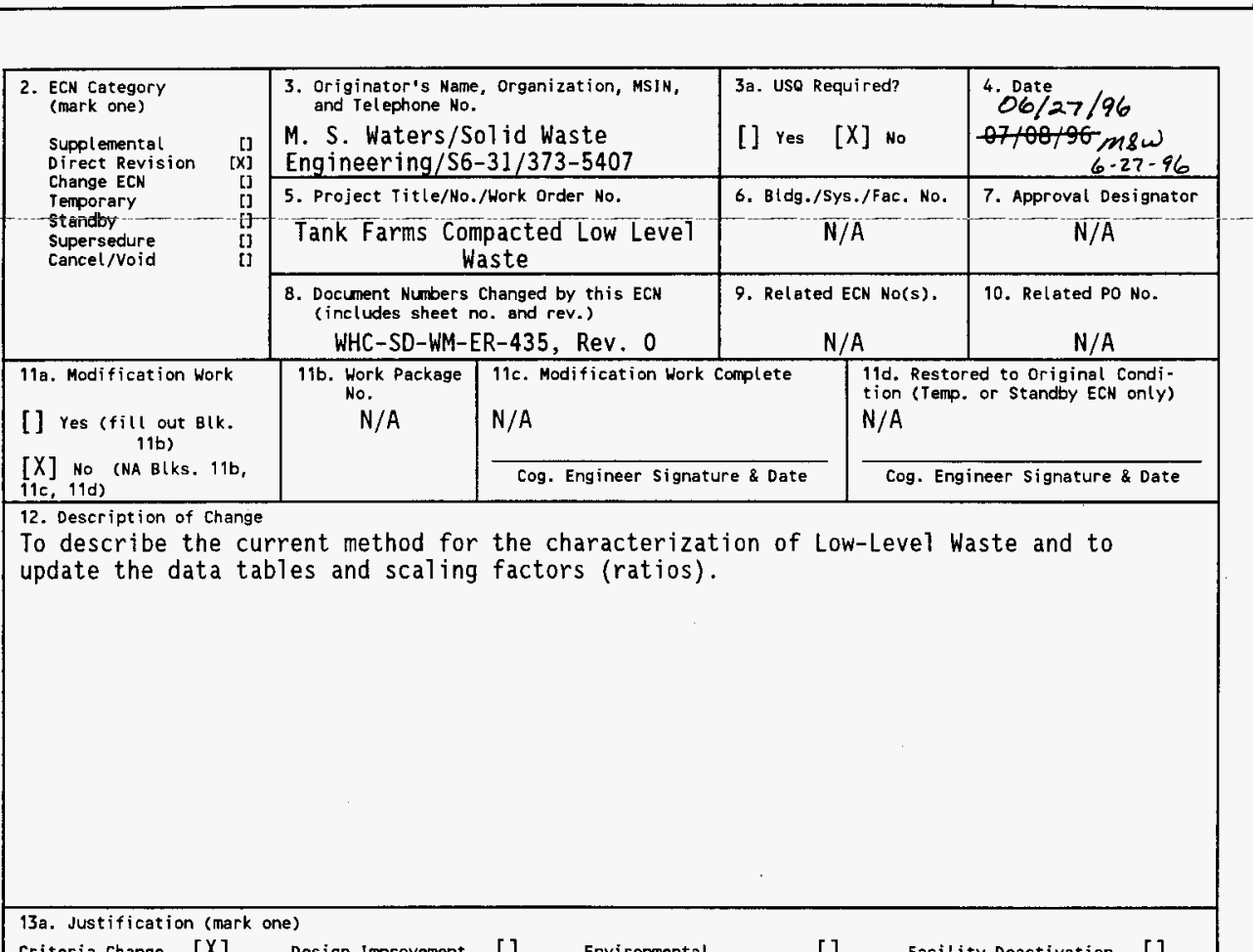

12. Description of Change

To describe the current method for the characterization of Low-Level Waste and to update the data tables and scaling factors (ratios).

13a. Justification (mark one)

\begin{tabular}{llllllll} 
Criteria Change & {$[X]$} & Design Improvenent & {[]} & Environmental & [] & Facility Deactivation [] \\
As-Found & {[]} & Facilitate Const & [] & Const. Error/Omission & [] & Design Error/Omission [] \\
\hline
\end{tabular}

13b. Justification Details

Method of interim storage and container inventory determination was modified. Additional sampling events yielded results to update data tables and recalculate scaling factors. Page change \#5 to WHC-EP-0063-4 added reporting criteria for mobile radionuclides.

14. Distribution (include name, MSIN, and no. of copies)

See attached distribution sheet.

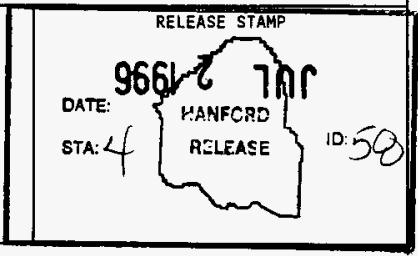

A-7900-013-2 (11/94) GEF095 


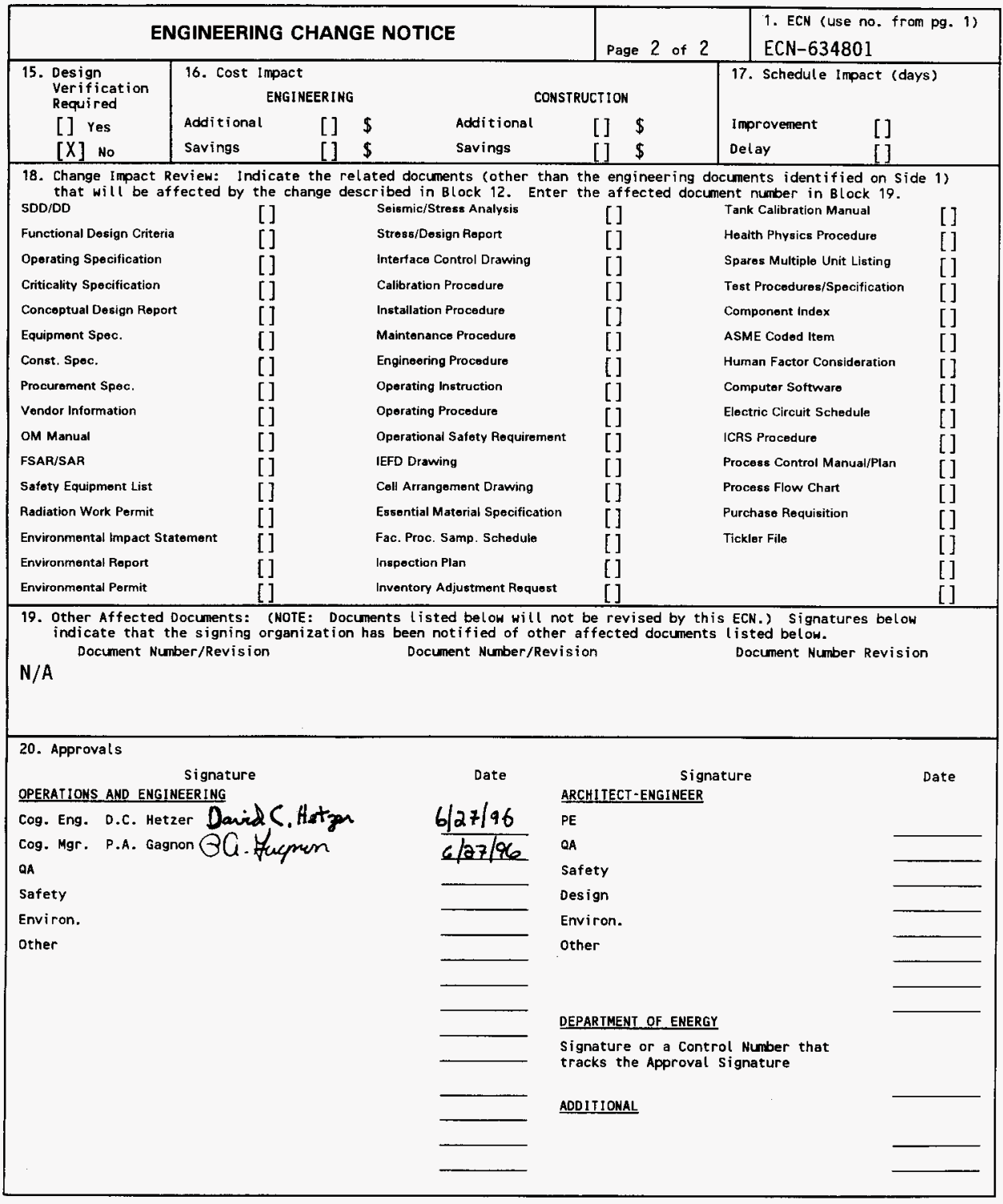




\section{Tank Farms Compacted Low Level Waste}

\section{Michael S. Waters}

Advanced Technologies Co., Inc., Richland, WA 99352

U.S. Department of Energy Contract DE-AC06-87RL10930

EDT/ECN: 634801

Org Code: 77520

B\&R Code: EW3130020
UC: 2000

Charge Code: N1380

Total Pages: 107

Key Words: Low Level Waste; Tank Farms; LLW; Compaction

Abstract: This report describes the process of Low Level Waste (LLW) volume reduction by compaction. Also included is the data used for characterization of LLW destined for compaction. Scaling factors (ratios) are formed based on data contained in this report.

TRADEMARK DISCLAIMER. Reference herein to any specific commercial product, process, or service by trade name, trademark, manufacturer. or otherwise. does not necessarily constitute or imply its endorsement. recommendation, or favoring by the United States Government or any agency thereof or its contractors or subcontractors.

Printed in the United States of America. To obtain copies of this document. contact: WHC/BCS Document Control Services. P.0. Box 1970. Mailstop H6-08. Richland WA 99352. Phone (509) 372-2420: Fax (509) 376-4989.
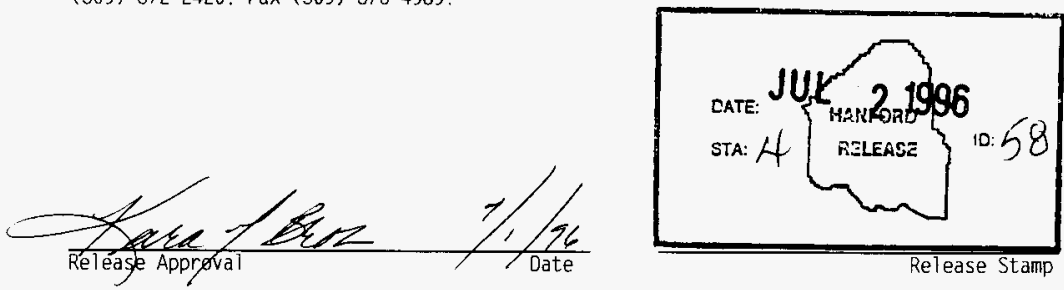

\section{Approved for Public Release}




\section{RECORD OF REVISION}

(1) Document Number

WHC-SD-WM-ER-435

Page 1

(2) Title

Tank Farms Compacted Low Level Waste

CHANGE CONTROL RECORD

\begin{tabular}{|c|c|c|c|}
\hline \multirow[t]{2}{*}{ Revision } & \multirow{2}{*}{ (4) Description of Change - Replace, Add, and Delete Pages } & \multicolumn{2}{|c|}{ Authorized for Release } \\
\hline & & (5) Cog. Engr. & (6) Cog. Mgr. \\
\hline 0 & $\begin{array}{l}\text { (7) Initially released } 08 / 18 / 95 \text { on EDT- } \\
611727\end{array}$ & M.S. Waters & P.A. Gagnon \\
\hline$R S 1$ & Incorporate per ECN-634801 & D.C. Hetzer & P.A. Gagnon \\
\hline & & & \\
\hline & & & \\
\hline & & & \\
\hline & & & 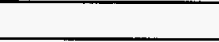 \\
\hline & & & \\
\hline & & & \\
\hline & & & \\
\hline & & & \\
\hline & & & \\
\hline & & & \\
\hline & & & \\
\hline & & & \\
\hline & & & \\
\hline & & & \\
\hline & & & \\
\hline & & & \\
\hline & & & \\
\hline & & & \\
\hline & & & \\
\hline & & & \\
\hline & & & \\
\hline & & & \\
\hline & & & \\
\hline & & & \\
\hline & & & \\
\hline & & & \\
\hline & & & \\
\hline & & & \\
\hline & & & \\
\hline & 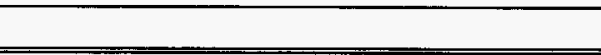 & & \\
\hline
\end{tabular}




\title{
TANK FARMS COMPACTED LOW LEVEL WASTE
}

\section{AUGUST 1995}

\author{
Revision 1 - June 1996
}

Prepared By:

Michael S. Waters

Advanced Technologies Co., Inc.

This is a extensive revision. Revision bars are excluded. 


\section{CONTENTS}

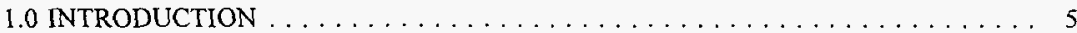

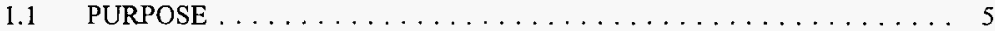

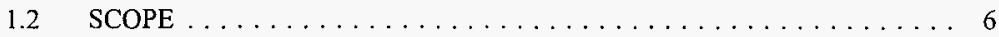

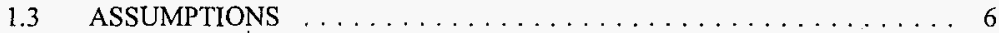

2.0 DESCRIPTION OF THE LLW WASTE STREAM $\ldots \ldots \ldots \ldots \ldots \ldots \ldots$

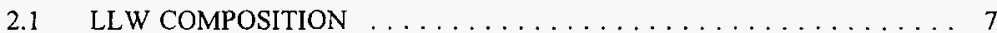

2.2 WASTE STREAM IDENTIFICATION $\ldots \ldots \ldots \ldots \ldots \ldots \ldots \ldots 7$

2.3 HISTORICAL LLW DATA $\ldots \ldots \ldots \ldots \ldots \ldots \ldots \ldots \ldots$

3.0 LLW SAMPLING AND ANALYSIS $\ldots \ldots \ldots \ldots \ldots \ldots \ldots \ldots \ldots \ldots$

3.1 DESTRUCTIVE SAMPLING AND ANALYSIS $\ldots \ldots \ldots \ldots \ldots \ldots 10$

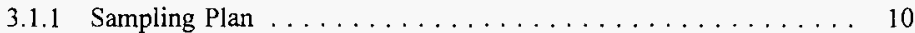

3.2 NON-DESTRUCTIVE ANALYSIS $\ldots \ldots \ldots \ldots \ldots \ldots \ldots \ldots \ldots 11$

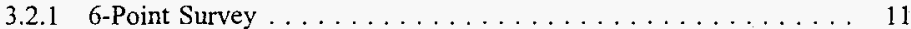

3.2 .2 PNNL Assay System $\ldots \ldots \ldots \ldots \ldots \ldots \ldots \ldots \ldots \ldots \ldots$

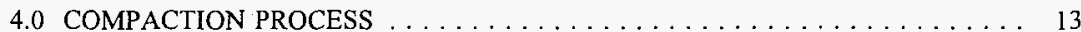

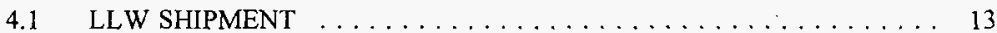

4.2 INITIAL COMPACTION $\ldots \ldots \ldots \ldots \ldots \ldots \ldots \ldots \ldots \ldots \ldots \ldots$

4.3 SUPERCOMPACTION $\ldots \ldots \ldots \ldots \ldots \ldots \ldots \ldots \ldots \ldots \ldots \ldots$

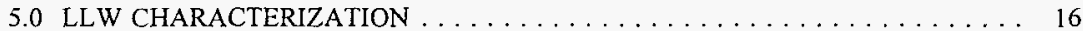

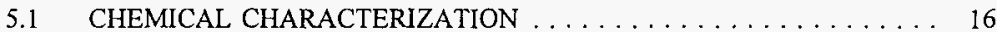

5.2 RADIOLOGICAL CHARACTERIZATION $\ldots \ldots \ldots \ldots \ldots \ldots \ldots$

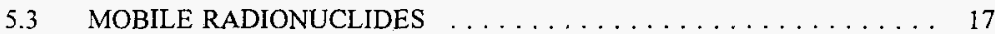

5.4 SCALING FACTORS (RATIOS) $\ldots \ldots \ldots \ldots \ldots \ldots \ldots \ldots \ldots$

6.0 CONCLUSIONS AND RECOMMENDATIONS $\ldots \ldots \ldots \ldots \ldots \ldots \ldots \ldots$

6.1 DATA NEEDS . . . . . . . . . . . . . . . . . . . . . . . . 19

6.2 SAMPLING AND ANALYSIS $\ldots \ldots \ldots \ldots \ldots \ldots \ldots \ldots \ldots$

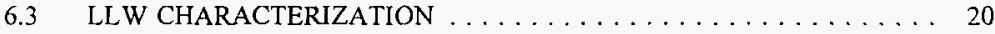

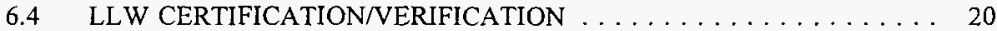

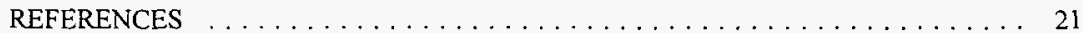

ATTACHMENT A $\ldots \ldots \ldots \ldots \ldots \ldots \ldots \ldots \ldots \ldots \ldots \ldots \ldots \ldots \ldots \ldots \ldots \ldots \ldots \ldots \ldots$

ATTACHMENT B $\ldots \ldots \ldots \ldots \ldots \ldots \ldots \ldots \ldots \ldots \ldots \ldots \ldots \ldots \ldots \ldots$ B-i

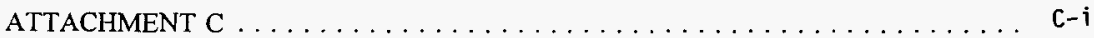

ATTACHMENT D . . . . . . . . . . . . . . . . . . . . . 


\section{LIST OF TERMS}

$\begin{array}{ll}\text { ATG } & \text { Allied Technology Group } \\ \text { EWO } & \text { Environmental Waste Operations } \\ \text { LLW } & \text { Low Level Waste } \\ \text { PIN } & \text { Package Identification Number } \\ \text { PNNL } & \text { Pacific Northwest National Laboratories } \\ \text { TRU } & \text { Transuranic Waste } \\ \text { WHC } & \text { Westinghouse Hanford Company } \\ \text { SWE } & \text { Solid Waste Engineering (Formerly Waste Shipment Engineering, WSE) }\end{array}$




\section{LIST OF DEFINITIONS}

Characterization. The determination of the waste composition and properties, whether by review of process knowledge, nondestructive examination or assay, or sampling and analysis, or a combination thereof, done for the purpose of determining appropriate storage, treatment, handling, transportation, and disposal requirements.

Low Level Waste. Radioactive waste that is not high level waste or transuranic waste, or byproduct material as defined in DOE Order $5820.2 \mathrm{~A}$.

Process Knowledge. The set of information that is used by individuals who are cognizant of the origin, use, and location of waste-generating materials and processes in sufficient detail so as to determine the identity of the waste.

Scaling Factors (Ratios). A multiplier that allows the inference of one difficult to measure radionuclide concentration from another that is more easily measured.

Segregation. The process of segregating (or keeping separate) individual waste types and/or forms in order to facilitate their cost-effective treatment and storage or disposal. 


\subsection{INTRODUCTION}

In January 1995, Westinghouse Hanford Company (WHC) commenced shipments of Tank Farm Low Level Waste (LLW) to the Allied Technology Group (ATG) for volume reduction utilizing supercompaction. The use of Sea-Land containers for the collection of LLW for shipment to ATG was established in November 1995. This report presents an overview of processes that identifies LLW destined for compaction and the waste characterization effort performed by Solid Waste Engineering (SWE).

There have been several significant changes in this process since the initial release of this document. The manner in which LLW is packaged and shipped to ATG, identified waste stream modifications, and the radiation limits for LLW destined for compaction have changed. There have been several additional sampling events. These samples were analyzed using a more extensive radionuclide list. These results are included to increase the analysis database and update scaling factors. Page change \#5 of the "Hanford Site Solid Waste Acceptance Criteria" manual, WHC-EP-0063-4, was issued in May 1996. This page change has added requirements for reporting certain mobile radionuclides for waste disposed in burial grounds.

All the ${ }^{99} \mathrm{Tc}$ data was not available to be included in this revision. A page change shall be done at a later date to include the ${ }^{99} \mathrm{Tc}$ results and conclusions.

\subsection{PURPOSE}

The purpose of this report is to provide the radionuclide data to characterize this waste stream for determining the inventory of waste containers. The process of LLW volume reduction by supercompaction and data utilization is also presented to provide a complete description of the method to ultimately determine the waste category for disposal. This report summarizes available information on identified components of the LLW waste stream, LLW historical data, sampling and analysis and LLW characterization data. Utilizing the above data, informed decisions can be made with regard to the ultimate disposition of LLW and the supercompaction process. Specific objectives of this report include;

- A description of the LLW waste stream components and an evaluation of the associated data.

- A description of the supercompaction process.

- Characterization of the LLW waste stream utilizing available sampling and analysis data.

- Determine scaling factors (ratios) for LLW. 


\subsection{SCOPE}

First, this report presents a description of the LLW composition and waste stream components considered for volume reduction. Next, a historical description of available LLW data is presented. This information includes data extracted from files used to ship LLW during 1993 and 1994. Next, a description of the LLW sampling and analysis followed by the process of waste preparation and supercompaction will be discussed. Finally, the characterization effort will be presented.

\subsection{ASSUMPTIONS}

The processes described in this report are based on current operating procedures and regulations with regards to the handling of LLW. LLW waste stream component identification and the composition of LLW is subject to change as the scope of work changes within Tank Farms.

This report will be reviewed annually, at a minimum, to evaluate its validity and will be revised as necessary. 


\subsection{DESCRIPTION OF THE LLW WASTE STREAM}

The purpose of this section is to provide an overview of the LLW waste stream and methods regarding the handling and disposition of identified LLW. This section will cover the composition of LLW, identification of the waste stream components and historical LLW data.

\section{$2.1 \quad$ LLW COMPOSITION}

Tank Farms LLW consists mostly of debris generated as a result of activities conducted within tank farms themselves. These activities include but are not limited to routine surveillance and maintenance, construction, demolition, facility upgrades, and general cleanup of tank farms. This debris is contaminated with radioactive isotopes mainly due to tank farm general area contamination. This debris has not come into contact with tank waste or chemical products regulated as dangerous waste (Ref. 4). At the current time, LLW dose rates are generally limited to $<10 \mathrm{mr} / \mathrm{hr}$ (Ref. 5).

The debris that makes up a majority of the accumulated LLW is cloth, rubber, paper, plastic, wood, concrete, and metal.

\subsection{WASTE STREAM IDENTIFICATION}

In the past there have been as many as seventeen (17) components identified for the LLW waste stream. This was the case at the initial release of this document. Since that time one component has been removed, 241-A. There are times when a waste stream component may become inactive and not produce LLW on a routine basis. There are various reasons that identified waste stream components may change or become inactive. As tank farms are declared clean and stable there is very little, if any, LLW generated at that point. The installation of remote surveillance equipment in tank farms requires fewer personnel entries thus reducing the amount of waste generated. Although these waste stream components may become inactive they are still monitored by Environmental Waste Operations (EWO) for collection of LLW. Table 2-1 illustrates the division of three (3) separate areas which are 200 East Tank Farms, 200 East Miscellaneous Areas, and 200 West Tank Farms. Subsequently each of these areas is broken down into locations of the collection point or box and then indicates which areas are serviced by each collection point.

There are instances when LLW containers will be issued for a particular work function to be conducted within tank farms. This may include removal of large equipment or materials or large scale farms cleanup where substantial quantities of LLW is expected to be generated. These container locations are tracked by EWO. 
A Sea-Land conex is maintained at the 209-E facility by Environmental Waste Operations. This is used for interim storage and as a shipping container for LLW that is destined for compaction. Routine pickup of the LLW waste from the collection points throughout Tank Farms is then returned to the 209-E Sea-Land container. This will be discussed further in Section 4.

\subsection{HISTORICAL LLW DATA}

A review of the files for previously generated LLW during the periods of 1993 to 1994 was conducted. The Package Identification Number files, or PIN files, contain information utilized for shipment of a LLW waste container. Pertinent information within these files could be used to aid in developing a history of the LLW waste stream and thus serve as documented process knowledge. Information collected was the PIN number for tracking purposes, the collection point and point of waste origin, weight and volume of the waste, the results of the 6-point radiation survey, results of the Pacific Northwest National Laboratory (PNNL) Assay and sample results if either were conducted, and the contents of the container as indicated on the inventory sheets. This information or data is summarized in Attachment A. 


\section{Table 2-1, COMPONENTS OF THE LOW LEVEL WASTE STREAM}

\begin{tabular}{|c|c|}
\hline Location of Collection Point & Areas Serviced \\
\hline \multicolumn{2}{|l|}{200 East Tank Farms } \\
\hline 241-AN & 241-AN \\
\hline 241-AP & 241-AP \\
\hline 241-AW & 241-AW \\
\hline 241-AY & 241-AX, AY, AZ \\
\hline $241-B$ & 241-B, 242-B, 242-BL \\
\hline $241-\mathrm{BX}$ & 241-BX, BY, BXR \\
\hline $241-\mathrm{C}$ & 241-C, 244-CR, 271-CR \\
\hline \multicolumn{2}{|l|}{200 East Miscellaneous } \\
\hline 242-A, EVAPORATOR & $242-\mathrm{A}$ \\
\hline $\begin{array}{l}\text { 204-AR, RAILCAR } \\
\text { UNLOADING FACILITY }\end{array}$ & 204-AR \\
\hline $\begin{array}{l}\text { 209-E, ENVIRONMENTAL } \\
\text { WASTE OPERATIONS }\end{array}$ & 209-E, REPACKAGING \\
\hline 244-A, LIFT STATION & $244-A$ \\
\hline \multicolumn{2}{|l|}{200 West Tank Farms } \\
\hline $241-S$ & 241-S, SX \\
\hline 241-SY & 241-SY \\
\hline $241-T$ & $241-\mathrm{T}$ \\
\hline 241-TX & 241-TX, TY \\
\hline $241-U$ & $241-U$ \\
\hline
\end{tabular}




\subsection{LLW SAMPLING AND ANALYSIS}

The purpose of this section is to provide an overview of sampling and analysis associated with LLW. Sampling and analysis is conducted to determine the identity and quantity of radionuclides present in LLW. This section will address destructive and nondestructive analysis.

\subsection{DESTRUCTIVE SAMPLING AND ANALYSIS}

Destructive sampling is performed by physically removing a portion of the waste for analysis. The sample is then placed into a container and transported to a laboratory for analysis. The sample matrix is selected which best represents the majority of the waste matrix from that particular waste stream component. The samples are collected in the field by EWO at each identified LLW waste stream component location. As discussed earlier in this report, the majority of the waste matrix to be sent for compaction is paper, cloth, rubber, and plastic. Small pieces of wood, concrete, and metal may also be included in compactable waste containers.

Typical analyses requested on LLW are Total Alpha (TA), Total Beta (TB), and Gamma Energy Analysis (GEA) although additional analyses may be requested. In order to perform the requested analyses on samples of this matrix, the laboratory will pretreat and prepare the sample using various laboratory techniques. After the requested analyses have been performed, the sample may then be archived at the laboratory for a period of time. If after review of the analysis results it is determined that additional analyses are required, the lab can be notified and the analysis completed on the remaining portion of the sample. If the results of the analyses are acceptable, the sample may then be discarded.

From a review of the historical data of LLW from within Tank Farms (Attachment A) it was evident that there was insufficient data to make informative decisions about the characterization of LLW destined for compaction. It was therefore decided to establish a plan to perform sampling and analysis of the waste stream components identified in Table 2-1. The results of these sampling and analysis efforts will establish a database to formulate characterization of the waste stream components.

\subsubsection{Sampling Plan}

For the initial release of this document a sampling plan was developed to identify radionuclides generated from the waste stream components identified in Table 2-1. The plan specified that three (3) separate sampling periods of approximately one week in duration would be conducted. The 222-S laboratory was contacted and they supplied sampling containers that had tare weight determined. This step would allow for the results to be 
reported in the units of $\rho \mathrm{Ci} / \mathrm{g}$. The first set of samples were collected $3 / 1 / 95$, the second set was collected $3 / 14 / 95$, and the third set was collected on $4 / 4 / 95$. There were also three blank samples sent to the lab to verify no cross-contamination was occurring. The blank samples indicated that no cross-contamination was occurring during laboratory analysis. This data is maintained in accordance with the WSE Data Management Plan.

Subsequent to the initial release of this document, four (4) additional sampling events have been conducted. Set \#4 was collected on $8 / 23 / 95$, \#5 was collected on $9 / 26 / 95$, \#6 was collected on $1 / 30 / 96$, and set \#7 was collected on $3 / 13 / 96$.

It should be noted that sample sets \#6 and \#7 were analyzed at the Pacific Northwest National Laboratory (PNNL). There were several reasons for having the samples analyzed at a different laboratory facility than had been used in the past. The initial issue of this document and the "Waste Shipment Engineering Radionuclide Characterization Program Plan", WHC-SD-WM-PLN-115, recommended the procurement of a laboratory facility that had the capabilities to perform analyses on samples utilizing an extended radionuclide list, meet our required sensitivities, and do so in a cost effective manner. Also a contributing factor to this endeavor was page change \#5 to "Hanford Site Solid Waste Acceptance Criteria", WHC-EP-0063-4. This page change included the requirements for reporting certain mobile radionuclide concentrations for waste disposed in burial grounds. The onsite laboratory facility does not have the capability to meet SWE needs for these radionuclides.

Results of the seven sets of samples collected are tabulated in Attachment B. It should be noted that the results from the first set of analysis are reported in $\rho \mathrm{Ci} / \mathrm{sample}$ instead of $\rho \mathrm{Ci} /$ gram. This was due to the samples being ashed and prepared for analysis prior to the net weight of the sample being determined. Although the units are different from the subsequent sets of analysis, the results are still valid for comparison purposes and may be useful in determining ratios discussed later.

\subsection{NON-DESTRUCTIVE ANALYSIS}

\subsubsection{6-Point Survey}

The 6-point survey is routinely conducted on all LLW containers. This involves a radiation survey meter to take contact radiation measurements on each side of the container. The highest radiation measurement detected on that side of the container is then recorded on a survey map. The results of the 6-point survey can be utilized to determine ${ }^{137} \mathrm{Cs}$ content of the container by using curves developed by the Criticallity and Shielding group. However, in order to consider the dose-rate to curie curves valid, two things should be known. First, it should be established that the predominant gamma emitting isotope of the waste is ${ }^{137} \mathrm{Cs}$. Secondly, the inventory of the container will be used to determine an approximate waste 
matrix density so that the appropriate dose-rate to curie curve can be utilized. The ${ }^{137} \mathrm{Cs}$ value determined by this method can then be used for characterization.

\subsubsection{PNNL Assay System}

The PNNL Assay System consists of gamma sensitive detectors and supporting computer equipment to identify and quantify the major gamma-emitting isotopes within the waste container. There are separate systems used for LLW boxes and LLW drums. The PNL Assay System also contains a passive-neutron system for the determination of TRU content of a waste container. The system has the advantage of being mobile which allows waste to be assayed prior to it being shipped from the Hanford Site and later at the compaction site after the initial compaction process. This is useful for verification and comparison purposes. The PNL Assay Systems are operated in accordance with approved PNNL procedures. (Ref. 7, 8) 


\subsection{COMPACTION PROCESS}

The purpose of this section is to provide an overview of the LLW compaction process. This section will address the current practice for shipment of LLW for compaction and an overview of the compaction process. An illustration of the compaction process is included as Figure 4-1.

\subsection{LLW SHIPMENT}

Prior to November 1995, tank farms LLW destined for compaction was packaged in the field in plastic bags and stored at one of the accumulation points until picked up by EWO personnel. The waste was then transported to the 209-E facility and accumulated in a waste container. The waste containers maintained at the 209-E facility were $4^{\prime} \times 4^{\prime} \times 8^{\prime}$ (or similar size) boxes that mimicked the accumulation areas identified in Table 2-1. Noncompactable waste is still segregated and placed in a separate container. When the containers were full they were prepared for shipment to the ATG compaction facility for volume reduction. The boxes were weighed to determine waste weight and an assay was performed by PNNL for characterization purposes.

In November 1995, EWO placed the Sea-Land containers into service on the Low Level Waste Pad at the 209-E facility. The Sea-Land container is $8^{\prime} \times 8^{\prime} \times 20^{\prime}$ and is used for intermediate storage and shipment of LLW to ATG. "Operate the Low Level Waste Pad", TO-100-056, is the procedure that directs operation of the low level waste pad. The LLW is still handled in the field and collected by EWO in the same manner as before November 1995. When the waste is returned to the 209-E facility, it is recorded on the "Farm Specific Log Sheet". Every tenth bag for that specific farm is placed in a "Sample LLW Box". If a waste bag is not to be placed in the "Sample LLW Box" it is placed in the Sea-Land container. When the Sea-Land container is full, the "Sample LLW Box" is assayed and the results used for characterization of the Sea-Land container. Transportation vehicles for shipment of the LLW containers is supplied by ATG. The brief summary of LLW shipments to the ATG facility are included as Attachment C.

\subsection{INITIAL COMPACTION}

When the waste shipment has been accepted at ATG, the bags of waste are removed from the container and prepared for compaction. At the first compaction process the bags are compacted into DOT $17 \mathrm{H}$ or UN1A2 painted steel 55 gallon drums. ATG is provided with instructions that specifies waste shall not be commingled. In other words, tank farms LLW will not be mixed with waste from another source during the compaction process. 
As stated earlier, PNNL performs assays on the LLW waste containers prior to shipment to ATG. For comparison purposes random assays were performed on compacted waste in the 55 gallon drums. These comparisons are summarized in Attachment D.

Initially the wooden boxes that were used for the LLW shipments to ATG were dismantled and compacted along with the waste that they contained. After the first two shipments it was determined that the boxes would be surveyed and reused when possible. This would improve the compaction ratios to the desired levels. With the use of the Sea-Land containers, the only wooden boxes used are for the "Sample LLW Box". When enough of the "Sample LLW Boxes" are amassed, they are shipped to ATG. The empty boxes are then returned to 209-E for reuse unless they are damaged at which point ATG would not return the box.

\subsection{SUPERCOMPACTION}

After the initial compaction of the waste into the 55 gallon drums, these drums then undergo supercompaction. This process results in the drums being reduced to "pucks" of varying dimensions. These "pucks" are then placed into larger overpacks meeting either DOT 7A Type A, UN1A2, or strong tight container specifications. These overpacks range in size from 64 to 85 gallons. ATG is then responsible for preparation of the shipping papers and return of the compacted waste to the Hanford Site. 
WHC-SD-WM-ER-435, Rev. 1

Page 15

Figure 4-1

\section{LLW VOLUME REDUCTION}

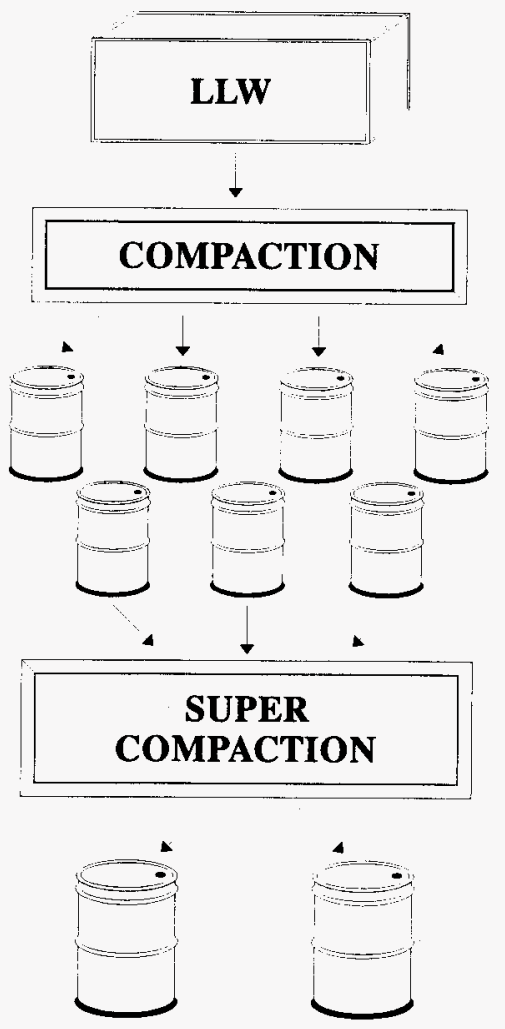




\subsection{LLW CHARACTERIZATION}

The purpose of this section is to provide an overview of the Characterization process for $\mathrm{LLW}$ destined for volume reduction.

\subsection{CHEMICAL CHARACTERIZATION}

Debris from tank farms is considered mixed waste if it contains tank waste. By definition, LLW waste is that fraction of waste generated throughout tank farms which does not contain tank waste (Ref. 4). Chemical products from various activities within tank farms may be present is LLW destined for compaction. Only nonregulated products, regulated products at concentrations below the regulatory limits, or empty containers of regulated products would be found in LLW.

\subsection{RADIOLOGICAL CHARACTERIZATION}

The radionuclide concentrations of LLW originates from the general area surface contamination from within tank farms. Debris is generated from various activities within tank farms such as maintenance and surveillance monitoring. Materials from this waste stream are segregated from mixed waste, i.e. tank contacted waste per procedure T0-100-052 (Ref. 5). A review of the data indicates that the major radionuclide constituents are ${ }^{137} \mathrm{Cs},{ }^{90} \mathrm{Sr} / \mathrm{Y},{ }^{99} \mathrm{Tc}$, and ${ }^{60} \mathrm{Co}$ with very limited quantities of alpha emitting radionuclides.

Characterization of the LLW waste stream components has relied on a combination of non-destructive and destructive analysis combined with a review of historical data. With the completion of seven destructive sampling and analysis events, an overall composition of the LLW waste stream components could be formulated. These sampling events were conducted over a time period of approximately one year. A review of the data indicates that the composition of the LLW waste stream components is fairly consistent. This data identifies ${ }^{137} \mathrm{Cs}$ as the primary gamma emitting radionuclide. With this being the case, scaling factors (ratios) are developed that will be utilized with a non-destructive assay method for characterization purposes and to determine waste category.

ATG is responsible for category determination of the compacted LLW that is returned to the Hanford Site. ATG, through their internal tracking system will determine the category of the compacted LLW based on the information supplied to them by the shipping organization. During the supercompaction process the compacted waste may occasionally reach Category 3 limits. Category 3 supercompacted LLW will be managed under its own Waste Specification Record. 


\subsection{MOBILE RADIONUCLIDES}

With the implementation of Page Change \#5 to WHC-EP-0063-4, the mobile radionuclides listed in Table 3-2 are required to be reported if they are in concentrations greater than the reporting limits. This table consists of 9 radionuclides, which are; ${ }^{3} \mathrm{H},{ }^{14} \mathrm{C}$, ${ }^{36} \mathrm{Cl},{ }^{79} \mathrm{Se},{ }^{99} \mathrm{Tc},{ }^{129} \mathrm{I},{ }^{187} \mathrm{Re}$, U (all), and ${ }^{237} \mathrm{~Np}$. Sample sets $\# 6 \& \# 7$ were sent to the PNNL laboratories for extensive radionuclide analysis which included analyses for the mobile radionuclides.

The analysis results for ${ }^{3} \mathrm{H},{ }^{14} \mathrm{C},{ }^{79} \mathrm{Se},{ }^{129} \mathrm{I}$, and ${ }^{187} \mathrm{Re}$ were returned as below detection limits for all samples submitted. Using best estimate values and sample calculations for a typical waste container, the Table 3-2 reporting levels would not be exceeded based on the reported detection limits for each radionuclide. There is currently no method developed for ${ }^{36} \mathrm{Cl}$ analysis on the types of matrix that makes up the majority of the LLW waste stream components. Using Appendix $\mathrm{K}$ of WHC-EP-0063-4 as an example for knowledge-of-source for plutonium manufacturing waste, Chlorine- 36 is not produced directly from fission and is not normally present in Hanford reactor fuel and cladding materials. Since the above mentioned radionuclides were below detection limits and ${ }^{36} \mathrm{Cl}$ can be justifiably omitted, these radionuclides will not be considered as present in the LLW waste stream components.

Of the remaining radionuclides of Table 3-2, analysis for $\mathrm{U}$ (total) was completed on sample sets \#4, \#5, \#6, and \#7. Analysis for ${ }^{237} \mathrm{~Np}$ was performed on sample sets \#6 \& \#7. The results of these analyses are used to calculate scaling factors and appear in Attachment $B$. The ratios for ${ }^{99} \mathrm{Tc}$ that appear in Attachment B are preliminary and should not be used at this time.

\subsection{SCALING FACTORS (RATIOS)}

WHC-EP-0063-4 allows the use of scaling factors to determine radionuclide concentrations. Scaling factors are used as multipliers that allows the inference of one difficult to measure radionuclide concentration from another that is more easily measured. It has been determined that there is sufficient sampling and analysis data available to generate scaling factors for each radionuclide of interest.

Applying standard statistical techniques to the sample data, scaling factors were calculated along with associated standard deviations and $95 \%$ confidence values. All data points were evaluated to determine their validity. Although the rejection of any one particular data point could be an indication of skewed data, Chauvenet's test for rejecting a reading can be utilized. Such is the case with 241-AN for the third sample set. This data point was rejected based on the test.

The final ratios and the supporting data for developing LLW ratios is included in Attachment $\mathrm{B}$ of this document. These ratios will be used in conjunction with the non- 
destructive assay results of the "Sample LLW Box" as discussed in section 4 and as discussed in the Waste Certification Summary for Supercompacted Low-Level Waste Debris (Ref. 4). As additional data becomes available, Attachment B shall be updated.

Once the final scaling factors were calculated, several test cases were performed to evaluate category determinations. Using best estimate values, the supercompacted LLW did stay within the Category 1 limits. 


\subsection{CONCLUSIONS AND RECOMMENDATIONS}

\subsection{DATA NEEDS}

The data and information gathered to support this document was done so to supply information particular to the LLW that is destined for compaction. A detailed evaluation was conducted and ratios determined. A continuing effort should be made to included information that would support accurate characterization of the LLW for compaction. Data will be organized and maintained in accordance with WHC-SD-WM-PLN-100, "Waste Shipment Engineering Data Management Plan" (Ref. 9).

\subsection{SAMPLING AND ANALYSIS}

The sample results of the LLW waste stream components indicate the presence of ${ }^{60} \mathrm{Co}$ in a large majority of the samples. Although ${ }^{137} \mathrm{Cs}$ is clearly the predominant gamma emitter, the fact that ${ }^{60} \mathrm{Co}$ is present should be evaluated. This is of particular concern when applying the current Dose-Rate-to-Curie curves for category determination. These curves only consider ${ }^{137} \mathrm{Cs}$ as the source. If ${ }^{137} \mathrm{Cs}$ represents $95 \%$ of the activity, the change to the Dose-Rate-toCure curves will be negligible due to the presence of other gamma emitters. If ${ }^{60} \mathrm{Co}$ and other gamma-ray emitters are present and contribute greater than $5 \%$ of the total gamma activity, new curves should be developed to reduce the overestimation of the radionuclide content. ${ }^{60} \mathrm{Co}$ should also be evaluated for potential alternative uses in the absence of ${ }^{137} \mathrm{Cs}$.

The current method for determining the ${ }^{137} \mathrm{Cs}$ activity and the application of scaling factors should be continued. This method uses the "Sample LLW Box" in conjunction with the PNNL assay system to determine the ${ }^{137} \mathrm{Cs}$ activity as well as activity for other gamma-ray emitting radionuclides, i.e., ${ }^{60} \mathrm{Co},{ }^{152} \mathrm{Eu},{ }^{154} \mathrm{Eu}$, and ${ }^{155} \mathrm{Eu}$. Scaling factors were not calculated for the "other" gamma-ray emitters due to the fact that the PNNL assayor will identify them if present. If another method is utilized to determine the ${ }^{137} \mathrm{Cs}$ activity, the scaling factors will need to be review, revised, and/or calculated as necessary.

Results of the Uranium analyses conducted on the LLW samples indicate the presence of Uranium in nearly all of the samples submitted. With the implementation of page change \#5 of WHC-EP-0063-4, specifically Table 3-2 reporting requirements, Uranium concentrations could be of concern at some point in time. Page change \#5 of WHC-EP-0063-4 specifies that "if the waste contains radionuclide concentrations that are derived from both the naturally occurring radioactivity in the media and man-made contaminants, the fraction derived from the naturally occurring source can be subtracted from the total inventory or concentration for comparison to the reporting limits". There have been extensive studies and evaluations performed on the environs of surrounding areas outside of tank farms. These evaluations have indicated the presence of natural levels of Uranium. An evaluation should be conducted 
to determine if in fact the concentrations of naturally occurring Uranium can be subtracted from the Uranium concentrations found in the LLW waste stream.

\subsection{LLW CHARACTERIZATION}

Each individual LLW waste stream component should continued to be monitored to ensure that it is acceptable to consolidate the waste. Waste streams that indicate they are not compatable with others may have to be isolated and maintained as a separate waste stream. As additional data becomes available, this document will be revised with the updated information.

\subsection{LLW CERTIFICATION/VERIFICATION}

Each LLW waste stream component should be monitored to ensure that the radionuclide content is not altered. Facility changes, process changes, or changes to the major matrix contributors of compactable LLW may indicate that scaling factors need to be verified and updated. At a minimum, reverification of the radionuclide content and scaling factor values should be conducted yearly. 


\section{REFERENCES}

(1) DOE Order 5820.2A, Radioactive Waste Management

(2) WHC-SD-WM-PLN-115, Rev 0, Waste Shipment Engineering Radionuclide Characterization Program Plan

(3) WHC-EP-0063, Rev 4, Hanford Site Solid Waste Acceptance Criteria

(4) WSRd Number 102-02, Waste Certification Summary, Supercompacted Low Level Waste Debris

(5) TO-100-052, Perform Waste Generation, Segregation, and Accumulation

(6) TO-100-056, Operate The Low Level Waste Pad

(7) DRM-001, Operation of the PNL Barrel Assay System to Measure Radioactive Emissions from 208-L Drums and Similar Containers

(8) SGS-002, Burial Box Assay by High-Resolution Gamma-Ray Spectrometry and/or Neutron Counting

(9) WHC-SD-WM-PLN-100, Waste Shipment Engineering Data Management Plan

(10) TO-100-010, Inspect, Load, and Ship Radioactive Waste To The Intermediate Holding Area 
WHC-SD-WM-ER-435, Rev. 1

Page A-i

\section{ATTACHMENT A}


TANK FARMS LLW

93/94 HISTORICAL. PIN FILES

\begin{tabular}{cccccccccc}
\hline PIN \# & Accum. Pt & $\begin{array}{c}\text { Point of } \\
\text { Origin }\end{array}$ & Weight & Volume & $6 \mathrm{pt}$ & ASSAY & SAMPLE & MAJOR CONTENTS \\
\hline & & $(\mathrm{Kg})$ & $\left(\mathrm{m}^{3}\right)$ & $(\mathrm{mr} / \mathrm{hr})$ & $\begin{array}{c}\mathrm{Cs}-137 \\
(\mathrm{pCl} / \mathrm{g})\end{array}$ & $\begin{array}{c}\text { Co-60 } \\
(\mathrm{pCl} / \mathrm{g})\end{array}$ & (see below)
\end{tabular}

$\begin{array}{llllll}93-106-04 & \text { AN } & \text { AN } & 282 & 4.0 & <0.5 \\ 93-106-04 & \text { AN } & \text { AN } & 388 & 4.0 & <0.2 \\ 94-143-02 & \text { AN } & \text { AN } & 369 & 4.0 & <0.5\end{array}$

$1,2,3,4$

$1,3,4,5,6$

94-143-02

AN

AN $\quad 369$

$<0.2$

$1,2,3,4,5$

1-Cloth, 2-Paper, 3-Plastic, 4-Rubber, 5-Metal, 6-Wood, 7-Concrete/Asphalt, 8-Vegetation, 9-Absorbent 
TANK FARMS LLW

93/94 HISTORICAL PIN FILES

\begin{tabular}{ccccccccc}
\hline PIN \# & Accum. Pt & $\begin{array}{c}\text { Point of } \\
\text { Origin }\end{array}$ & Weight & Volume & $6 \mathrm{pt}$ & ASSAY & SAMPLE & MAJOR CONTENTS \\
\hline & & $(\mathrm{Kg})$ & $\left(\mathrm{m}^{3}\right)$ & $(\mathrm{mr} / \mathrm{hr})$ & $\begin{array}{c}\text { Cs-137 } \\
\text { (pCi/g) }\end{array}$ & $\begin{array}{c}\text { Co-60 } \\
\text { (pCi/g) }\end{array}$ & (see below)
\end{tabular}

93-105-09

93-105-10
AP

AP

296

396.9
4.0

$<0.5$

$<0.5$

$<0.67$

0.70
$1,2,3,4,5,9$

$1,2,3,4,5$

1-Cloth, 2-Paper, 3-Plastic, 4-Rubber, 5-Metal, 6-Wood, 7-Concrete/Asphalt, 8-Vegetation, 9-Absorbent 
TANK FARMS LLW

93/94 HISTORICAL PIN FILES

\begin{tabular}{rcccccccrr}
\hline PIN\# & Accum. Pt & $\begin{array}{c}\text { Point of } \\
\text { Origin }\end{array}$ & Weight & Volume & $6 \mathrm{pt}$ & ASSAY & SAMPLE & MAJOR CONTENTS \\
\hline & & & (Kg) & $\left(\mathrm{m}^{3}\right)$ & $(\mathrm{mr} / \mathrm{hr})$ & $\begin{array}{c}\text { Cs-137 } \\
\text { (pCi/g) }\end{array}$ & $\begin{array}{c}\text { Co-60 } \\
\text { (pCi/g) }\end{array}$ & Tot. Act. & (see below) \\
& & & & & & & & & \\
$93-105-18$ & AW & AW & 153 & 4.0 & $<0.2$ & & & $1,2,3,4,7,8$ \\
$93-105-19$ & AW & AW & 279 & 4.0 & $<0.5$ & 75.0 & 2.60 & & $2,3,4,5$ \\
$93-215-03$ & AW & AW & 323 & 4.0 & $<0.5$ & & & & $1,2,3,4$ \\
$94-208-02$ & AW & AW & 396 & 4.0 & 0.5 & & & & $1,2,3,4,5,6$
\end{tabular}


TANK FARMS LLW

93/94 HISTORICAL PIN FILES

\begin{tabular}{|c|c|c|c|c|c|c|c|c|c|}
\hline \multirow[t]{2}{*}{ PIN \# } & \multirow[t]{2}{*}{ Accum. Pt } & \multirow{2}{*}{$\begin{array}{c}\text { Point of } \\
\text { Origin }\end{array}$} & \multirow{2}{*}{$\frac{\text { Weight }}{\text { (Kg) }}$} & \multirow{2}{*}{$\frac{\text { Volume }}{\left(\mathrm{m}^{3}\right)}$} & \multirow{2}{*}{$\frac{6 \mathrm{pt}}{(\mathrm{mr} / \mathrm{hr})}$} & \multicolumn{2}{|c|}{ ASSAY } & \multirow{2}{*}{$\begin{array}{c}\text { SAMPLE } \\
\begin{array}{c}\text { Tot. Act. } \\
\text { (pCi/g) }\end{array}\end{array}$} & \multirow{2}{*}{$\begin{array}{c}\text { MAJOR CONTENTS } \\
\text { (see below) }\end{array}$} \\
\hline & & & & & & $\begin{array}{r}C s-137 \\
\quad p\end{array}$ & Co-60 & & \\
\hline $93-091-02$ & AY & AP,AW,AY & 292 & 4.0 & $<0.5$ & & & & $1,2,3,4$ \\
\hline $93-102-02$ & AY & C,AN,AY & 321 & 4.0 & $<0.5$ & & & & $1,2,3,4$ \\
\hline $93-105-12$ & AY & A & 320 & 4.0 & & & & & $1,2,3,4,9$ \\
\hline $93-105-13$ & AY & $A$ & 978 & 4.0 & $<0.5$ & & & & $3,4,5,6$ \\
\hline $93-109-08$ & $A Y$ & $A$ & 452 & 4.0 & & & & $<50$ & $1,2,3$ \\
\hline $93-109-09$ & AY & AY & 669 & 4.0 & $<0.2$ & & & & $1,2,3,4,5,6$ \\
\hline $93-109-14$ & AY & $A_{1} A Y$ & 778 & 40 & & & & & 5,6 \\
\hline $93-110-01$ & AY & $A, A Y, A Z$ & 328 & 4.0 & $<0.2$ & & & & $1,2,3,4$ \\
\hline $93-111-01$ & AY & AY & 595 & 4.0 & $<0.2$ & & & & $5,6,7$ \\
\hline $93-111-13$ & $A Y$ & $A, A X, A Y$ & 867 & 4.0 & $<0.2$ & & & & $1,3,5,6$ \\
\hline $93-127-06$ & AY & A & 244 & 4.0 & $<0.2$ & & & & $1,2,3,4,5$ \\
\hline $93-130-01$ & AY & $A$ & 573 & 4.0 & 0.5 & 270 & 0.7 & & $1,2,3,4,5,6$ \\
\hline $93-144-01$ & AY & AY & 37.2 & 0.2 & $<0.5$ & 1.1 & $<1.1$ & $<50$ & 9 \\
\hline $93-144-02$ & AY & AY & 31.3 & 0.2 & $<0.5$ & 1.3 & $<1.6$ & $<50$ & 9 \\
\hline $93-144-03$ & AY & $A Y$ & 36.7 & 0.2 & $<0.5$ & 1.6 & $<1.2$ & $<50$ & 9 \\
\hline $93-165-01$ & AY & A,AY & 604 & 4.0 & $<0.2$ & & & & $1,2,3,4$ \\
\hline $93-221-01$ & AY & A & 367 & 4.0 & $<0.2$ & & & & $1,2,3,4,8$ \\
\hline $93-316-01$ & AY & AY & 415 & 4.0 & $<0.5$ & 270 & 0.91 & & $1,2,3,4,5$ \\
\hline $93-349-04$ & AY & $A, A X, A Z$ & 300 & 4.0 & $<0.5$ & 170 & 5.4 & & $1,2,3,4$ \\
\hline $94-089-02$ & AY & & 269 & 4.0 & $<0.5$ & 22 & 2.1 & & $1,2,3,4$ \\
\hline $94-104-02$ & AY & AY & 49.0 & 0.2 & $<0.5$ & & & & 5,9 \\
\hline
\end{tabular}


TANK FARMS LLW

93/94 HISTORICAL PIN FILES

\begin{tabular}{|c|c|c|c|c|c|c|c|c|}
\hline PIN \# & Accum. Pt & $\begin{array}{l}\text { Point of } \\
\text { Origin }\end{array}$ & Weight & Volume & $6 \mathrm{pt}$ & ASSAY & SAMPLE & MAJOR CONTENTS \\
\hline & & & $(\mathrm{Kg})$ & $\left(m^{3}\right)$ & $(\mathrm{mr} / \mathrm{hr})$ & $\begin{array}{c}\mathrm{Cs}-137 \quad \mathrm{Co-60} \\
(\mathrm{pCi} / \mathrm{g})\end{array}$ & $\begin{array}{l}\text { Tot. Act. } \\
\text { (pCi/g) }\end{array}$ & (see below) \\
\hline $94-159-03$ & AY & & 383 & 4.0 & $<0.5$ & & & $1,2,3,4,5$ \\
\hline $94-159-04$ & AY & & 382 & 4.0 & $<0.5$ & & & $1,2,3,4,5$ \\
\hline $94-158-02$ & AY & $701-A$ & 301 & 0.2 & $<0.5$ & & & 7 \\
\hline $94-158-03$ & AY & $701-A$ & 265 & 0.2 & $<0.5$ & & & 7 \\
\hline $94-165-04$ & AY & 701-A & 292 & 0.2 & $<0.5$ & & & 7, SOIL \\
\hline $94-165-05$ & AY & $701-A$ & 292 & 0.2 & $<0.5$ & & $<50$ & SOIL \\
\hline $94-173-04$ & AY & $701-A$ & 315 & 0.2 & $<0.5$ & & & 7, SOIL \\
\hline $94-173-05$ & $A Y$ & $701-A$ & 310 & 0.2 & $<0.5$ & & $<50$ & SOIL \\
\hline $94-174-01$ & AY & $701-A$ & 319 & 0.2 & $<0.5$ & & $<50$ & SOIL \\
\hline $94-174-02$ & AY & $701-A$ & 333 & 0.2 & $<0.5$ & & $<50$ & SOIL \\
\hline $94-174-03$ & AY & 701-A & 328 & 0.2 & $<0.5$ & & $<50$ & SOIL \\
\hline $94-208-04$ & AY & & 255 & 4.0 & $<0.5$ & & & $1,2,3,4,5$ \\
\hline $94-271-03$ & AY & & 405 & 4.0 & $<0.5$ & & & $1,2,3,4,5,6$ \\
\hline $94-293-12$ & AY & 701-A & 142 & 0.2 & $<0.5$ & & $<50$ & 7 \\
\hline
\end{tabular}


TANK FARMS LLW

93/94 HISTORICAL PIN FILES

\begin{tabular}{cccccccccc}
\hline PIN \# & Accum. Pt & $\begin{array}{c}\text { Point of } \\
\text { Origin }\end{array}$ & Weight & Volume & $6 \mathrm{pt}$ & ASSAY & SAMPLE & MAJOR CONTENTS \\
\hline & & & $(\mathrm{Kg})$ & $\left(\mathrm{m}^{3}\right)$ & $(\mathrm{mr} / \mathrm{hr})$ & $\begin{array}{c}\mathrm{Cs}-137 \\
(\mathrm{pCi} / \mathrm{g})\end{array}$ & $\begin{array}{c}\text { Co-60 } \\
(\mathrm{pCi} / \mathrm{g})\end{array}$ & (see below) \\
$93-105-23$ & B & B & 188 & 4.0 & $<0.5$ & $<1.6$ & 1.70 & & $1,2,3,4,9$ \\
$93-105-24$ & B & B & 87 & 4.0 & $<0.5$ & & & & $1,2,3,4,5,7,8,9$
\end{tabular}


ATTACHMENT A

TANK FARMS LLW

93/94 HISTORICAL PIN FILES

\begin{tabular}{|c|c|c|c|c|c|c|c|c|}
\hline PIN\# & Accum. Pt & $\begin{array}{l}\text { Point of } \\
\text { Origin }\end{array}$ & Weight & Volume & $6 \mathrm{pt}$ & ASSAY & SAMPLE & MAJOR CONTENTS \\
\hline & & & $(\mathrm{Kg})$ & $\left(m^{3}\right)$ & $(\mathrm{mr} / \mathrm{hr})$ & $\begin{array}{c}\text { Cs-137 } \\
(\mathrm{pC} / \mathrm{g})\end{array}$ & $\begin{array}{l}\text { Tot. Act. } \\
(\mathrm{pCi} / \mathrm{g})\end{array}$ & (see below) \\
\hline $93-105-06$ & $B X$ & $\mathrm{BX}, \mathrm{BY}$ & 286 & 4.0 & & & & $1,2,3,6$ \\
\hline $93-105-07$ & $\mathrm{BX}$ & $\mathrm{BX}, \mathrm{BY}$ & 462 & 4.0 & $<0.5$ & & & $1,2,3,4,5,6$ \\
\hline $93-111-02$ & $\mathrm{BX}$ & BX & 1039 & 4.0 & $<0.5$ & & & $5,6,9$ \\
\hline $93-111-03$ & $\mathrm{BX}$ & BX & 1289 & 4.0 & $<0.5$ & & & 5,7 \\
\hline $93-112-02$ & $\mathrm{BX}$ & $B X, B Y$ & 539 & 4.0 & $<0.2$ & & & $1,2,3,4,5,6$ \\
\hline $93-112-03$ & $\mathrm{BX}$ & BX & 381 & 4.0 & & & & $1,2,3,4$ \\
\hline $93-116-09$ & $B X$ & BX,BY & 286 & 4.0 & $<0.2$ & & & $1,2,3,4$ \\
\hline $93-116-10$ & $B X$ & BX & 599 & 4.0 & $<0.2$ & & & $1,2,3,4,5,6$ \\
\hline $93-118-01$ & $\mathrm{BX}$ & $B X, B Y$ & 523 & 4.0 & $<0.5$ & & & $1,2,3,5,6$ \\
\hline $93-118-05$ & $\mathrm{BX}$ & BX & 224 & 4.0 & $<0.5$ & & & $1,2,3,5,6$ \\
\hline $93-120-03$ & $\mathrm{BX}$ & $B X$ & 615 & 4.0 & $<0.5$ & & & 6 \\
\hline $93-120-04$ & $\mathrm{BX}$ & $\mathrm{BX}$ & 1532 & 4.0 & $<0.2$ & & & 5,6 \\
\hline $93-124-02$ & $\mathrm{BX}$ & $\mathrm{BX}$ & 903 & 4.0 & $<0.2$ & & & $2,3,4,5$ \\
\hline $93-126-05$ & $\mathrm{BX}$ & BX & 1005 & 4.0 & $<0.2$ & & & $2,3,5,6$ \\
\hline $93-126-06$ & $B X$ & $B X$ & 1596 & 4.0 & $<0.2$ & & & $3,5,6$ \\
\hline $93-126-07$ & $B X$ & $B X$ & 675 & 4.0 & $<0.2$ & & & 6 \\
\hline $93-134-01$ & $B X$ & $\mathrm{BX}, \mathrm{BY}$ & 760 & 4.0 & $<0.2$ & & & $1,2,3,4,5,6,7$ \\
\hline $93-134-03$ & $\mathrm{BX}$ & $B X, B Y$ & 651 & 4.0 & 1 & & & $1,2,3,4,5,6$ \\
\hline $93-190-01$ & $\mathrm{BX}$ & $B X, B Y$ & 369 & 4.0 & $<0.5$ & & & $1,2,3,4$ \\
\hline $93-200-02$ & $\mathrm{BX}$ & $\mathrm{BX}, \mathrm{BY}$ & 481 & 4.0 & $<0.2$ & & & $1,2,3,4,5$ \\
\hline $93-202-05$ & $B X$ & $B X$ & 553 & 4.0 & $<0.2$ & & & 6,9 \\
\hline
\end{tabular}


TANK FARMS LLW

93/94 HISTORICAL PIN FILES

\begin{tabular}{|c|c|c|c|c|c|c|c|c|c|}
\hline PIN\# & Accum. Pt & $\begin{array}{c}\text { Point of } \\
\text { Origin }\end{array}$ & Weight & Volume & $6 \mathrm{pt}$ & $\overline{\mathrm{AS}}$ & & SAMPLE & MAJOR CONTENTS \\
\hline & & & $(\mathrm{Kg})$ & $\left(m^{3}\right)$ & $(\mathrm{mr} / \mathrm{hr})$ & $\begin{array}{r}\text { Cs-137 } \\
\text { (pC }\end{array}$ & $\begin{array}{l}\text { Co- } 60 \\
\text { g) }\end{array}$ & $\begin{array}{c}\text { Tot. Act. } \\
(\mathrm{pCi} / \mathrm{g})\end{array}$ & (see below) \\
\hline 93-202-06 & $\mathrm{BX}$ & $8 X$ & 569 & 4.0 & $<0.2$ & & & & $4,6,9$ \\
\hline $93-204-01$ & $\mathrm{BX}$ & $B X$ & 583 & 4.0 & $<0.5$ & & & & 6,9 \\
\hline $93-204-02$ & $B X$ & $B X$ & 551 & 4.0 & $<0.5$ & & & & 6,9 \\
\hline $93-204-03$ & $B X$ & $B X$ & 562 & 4.0 & $<0.5$ & & & & 6,9 \\
\hline $93-210-02$ & $B X$ & $B X$ & 553 & 4.0 & $<0.2$ & & & & $4,6,9$ \\
\hline $93-210-03$ & $B X$ & $B X$ & 169 & 4.0 & $<0.2$ & & & & $1,2,3,4,5$ \\
\hline $93-210-04$ & $B X$ & $B X$ & 540 & 4.0 & $<0.2$ & & & & $5,6,7,9$ \\
\hline $93-210-05$ & $B X$ & $\mathrm{BX}$ & 689 & 4.0 & $<0.2$ & & & & $1,2,3,4,5$ \\
\hline $93-215-01$ & $B X$ & $B X$ & 1448 & 4.0 & $<0.5$ & & & & $2,4,5,6$ \\
\hline $93-277-01$ & $B X$ & $B X, B Y$ & 215 & 4.0 & $<0.5$ & & & & $1,2,3,4,6$ \\
\hline $93-285-01$ & $\mathrm{BX}$ & $\mathrm{BX}, \mathrm{BY}$ & 315 & 4.0 & $<0.5$ & & & & $1,2,3,4,5$ \\
\hline $94-035-01$ & $B X$ & $\mathrm{BX}, \mathrm{BY}$ & 188 & 4.0 & 0.7 & 12000 & 4.0 & & $1,2,3,4$ \\
\hline $94-074-02$ & $B X$ & BXR & 1094 & 6.7 & $<0.5$ & & & & $3,5,6$ \\
\hline $94-108-02$ & $\mathrm{BX}$ & BXR & 492 & 4.0 & $<0.5$ & 130 & 0.6 & & $1,2,3,4,5$ \\
\hline $94-136-03$ & $\mathrm{BX}$ & $\mathrm{BX}$ & 451 & 4.0 & $<0.5$ & 610 & 0.4 & & $1,2,3,4,5$ \\
\hline $94-151-01$ & $B X$ & $B X$ & 223 & 4.0 & $<0.5$ & & & & $1,2,3,4,5,6$ \\
\hline $94-178-01$ & $B X$ & $B X$ & 314 & 4.0 & $<0.5$ & & & & $1,2,3,4,5$ \\
\hline $94-206-01$ & $B X$ & $B X$ & 478 & 4.0 & $<0.5$ & & & & $1,2,3,4,5$ \\
\hline $94-252-01$ & $B X$ & $\mathrm{BX}$ & 292 & 4.0 & $<0.5$ & & & & $1,2,3,4,5$ \\
\hline $94-270-09$ & $B X$ & $\mathrm{BX}$ & 392 & 4.0 & $<0.5$ & & & & $1,2,3,4,5$ \\
\hline
\end{tabular}


ATTACHMENT A

WHC-SD-WM-ER-435, Rev. 1

Page A-9 of 21

TANK FARMS LLW

93/94 HISTORICAL PIN FILES

\begin{tabular}{|c|c|c|c|c|c|c|c|c|c|}
\hline PIN \# & Accum. Pt & Point of & Weight & Volume & $6 \mathrm{pt}$ & ASS & $\overline{\mathrm{A} Y \bar{Y}}$ & SAMPLE & MAJOR CONTENTS \\
\hline & & & $(\mathrm{Kg})$ & $\left(\mathrm{m}^{3}\right)$ & $(\mathrm{mr} / \mathrm{hr})$ & $\begin{array}{r}\mathrm{Cs}-137 \\
\quad(\mathrm{pC}\end{array}$ & ${ }_{i / g)}^{C 0-60}$ & $\begin{array}{l}\text { Tot. Act } \\
\text { (pCi/g) }\end{array}$ & (see below) \\
\hline $93-105-28$ & C & C & 354 & 4.0 & & & & & $1,2,3,4$ \\
\hline $93-105-29$ & C & C & 660 & 4.0 & & & & & $1,2,3,4,5,6$ \\
\hline $93-110-19$ & $\mathrm{C}$ & C & 204 & 4.0 & $<0.5$ & 221.0 & 1.80 & & $1,2,3,4$ \\
\hline $93-110-20$ & C & C & 437 & 4.0 & $<0.5$ & & & & $3,4,5,6$ \\
\hline $93-111-04$ & c & C & 394 & 4.0 & $<0.5$ & & & & $1,3,4,5,6$ \\
\hline 93-111-09 & C & C & 805 & 4.0 & $<0.2$ & & & & $3,5,6$ \\
\hline $93-111-10$ & C & C & 669 & 4.0 & $<0.5$ & & & & $3,4,5,6,7,9$ \\
\hline $93-113-04$ & C & C & 218 & 4.0 & $<0.2$ & & & & $1,2,3,4,9$ \\
\hline $93-113-05$ & C & C & 762 & 4.0 & $<0.2$ & & & & $1,2,3,4,5,6$ \\
\hline $93-167-02$ & C & C & 184 & 4.0 & $<0.2$ & & & & $3,8,9$ \\
\hline $93-207-01$ & $\mathrm{C}$ & C & 132 & 4.0 & $<0.5$ & & & & FOAM, 9 \\
\hline $93-207-02$ & C & $106-C$ & 152 & 4.0 & $<0.5$ & 1700 & $<1.2$ & & FOAM, 9 \\
\hline $93-209-01$ & $\mathrm{C}$ & $106-C$ & 274 & 4.0 & $<0.5$ & & & & $1,2,3,4$ \\
\hline $93-270-01$ & $\mathrm{c}$ & c & 255 & 4.0 & $<0.2$ & & & & $1,2,3,4,9$ \\
\hline $93-278-01$ & C & C & 413 & 4.0 & 5.0 & 17000.0 & $<0.56$ & 144000.0 & $2,3,4,6,8$ \\
\hline $93-278-01$ & C & c & SAM & E DATA- & $\ldots$ & (GEA): $:^{137} \mathrm{Cs}$ & 9.23E05, & Sr: $2.85 E 02$ & \\
\hline $93-312-01$ & $\mathrm{C}$ & C & 169 & 4.0 & $<0.5$ & & & & $1,2,3,4,9$ \\
\hline $93-362-01$ & $\mathrm{C}$ & c & 233 & 4.0 & $<0.5$ & & & & $1,2,3,4,9$ \\
\hline $94-019-02$ & $\mathrm{C}$ & $\mathrm{C}$ & 483 & 4.0 & $<0.5$ & 270 & 0.69 & & $1,2,3,4,5,9$ \\
\hline $94-048-01$ & C & C & 306 & 4.0 & $<0.5$ & 190 & 1.7 & & $1,2,3,4,9$ \\
\hline $94-088-02$ & C & C & 319 & 4.0 & $<0.5$ & & & & $1,2,3,4,5,6,9$ \\
\hline
\end{tabular}


TANK FARMS LLW

93/94 HISTORICAL PIN FILES

\begin{tabular}{|c|c|c|c|c|c|c|c|c|c|}
\hline \multirow[t]{2}{*}{ PIN\# } & \multirow[t]{2}{*}{ Accum. Pt } & \multirow{2}{*}{$\begin{array}{l}\text { Point of } \\
\text { Origin }\end{array}$} & \multirow{2}{*}{ Weight } & \multirow{2}{*}{$\begin{array}{c}\text { Volume } \\
\left(\mathrm{m}^{3}\right)\end{array}$} & \multirow{2}{*}{$\begin{array}{c}6 \mathrm{pt} \\
(\mathrm{mr} / \mathrm{hr})\end{array}$} & \multicolumn{2}{|c|}{ ASSAY } & \multirow{2}{*}{$\begin{array}{c}\text { SAMPLE } \\
\text { Tot. Act. } \\
\text { (pCi/g) }\end{array}$} & \multirow{2}{*}{$\begin{array}{c}\text { MAJOR CONTENTS } \\
\text { (see below) }\end{array}$} \\
\hline & & & & & & $\mathrm{Cs}-137$ & $\begin{array}{l}\text { Co-60 } \\
\text { g) }\end{array}$ & & \\
\hline $94-115-03$ & C & 244-C & 198 & 0.2 & $<0.5$ & 460 & 0.53 & & $1,2,3,4,9$ \\
\hline $94-129-01$ & C & C & 396 & 4.0 & $<0.5$ & 87 & 0.35 & & $1,2,3,4,5,9$ \\
\hline $94-137-02$ & C & $\mathrm{C}$ & 460 & 4.0 & $<0.5$ & 610 & 0.38 & & $1,2,3,4,5,9$ \\
\hline $94-147-02$ & C & C & 329 & 4.0 & $<0.5$ & & & & $1,2,3,4,5,6,9$ \\
\hline $94-173-02$ & $\mathrm{C}$ & C & 292 & 4.0 & $<0.5$ & & & & $1,2,3,4,5,9$ \\
\hline $94-193-02$ & C & C & 339 & 4.0 & $<0.5$ & & & & $1,2,3,4,5,9$ \\
\hline $94-242-01$ & $\mathrm{C}$ & $\mathrm{C}$ & 373 & 4.0 & $<0.5$ & & & & $1,2,3,4,5,9$ \\
\hline $94-270-01$ & C & C & 369 & 4.0 & $<0.5$ & & & & $1,2,3,4,5,6,9$ \\
\hline $94-292-02$ & C & $c$ & 632 & 4.0 & $<0.5$ & & & & $1,2,3,4,5,9$ \\
\hline
\end{tabular}


TANK FARMS LLW

93/94 HISTORICAL PIN FILES

\begin{tabular}{|c|c|c|c|c|c|c|c|c|c|}
\hline PIN \# & Accum. $\mathrm{Pt}$ & Point of & Weight & Volume & $6 \mathrm{pt}$ & & & SAMPLE & MAJOR CONTENTS \\
\hline & & & $(\mathrm{Kg})$ & $\left(\mathrm{m}^{3}\right)$ & $(\mathrm{mr} / \mathrm{hr})$ & Cs-137 & Co-60 & Tot. Act. & (see below) \\
\hline & & & & & & & & $(\mathrm{pCi} / \mathrm{g})$ & \\
\hline $93-113-07$ & $242-A$ & $242-A$ & 790 & 4.0 & $<0.2$ & & & & 5,6 \\
\hline $93-118-04$ & $242-A$ & $242-A$ & 1016 & 4.0 & $<0.5$ & & & & 1,456 \\
\hline $93-127-03$ & $242-A$ & $242-A$ & 292 & 4.0 & $<0.5$ & & & & $1,2,3,4$ \\
\hline $93-127-04$ & 242-A & 242-A & 360 & 4.0 & $<0.2$ & & & & $1,2,3,5$ \\
\hline $93-200-01$ & $242-A$ & $242-A$ & 426 & 4.0 & $<0.5$ & & & & $1,2,3,4,5$ \\
\hline $94-108-01$ & 242-A & $242-A$ & 451 & 4.0 & $<0.5$ & & & & $1,2,3,4,5$ \\
\hline $94-122-01$ & 242-A & $242-A$ & 492 & 4.0 & $<0.5$ & & & & $1,2,3,4,5,8$ \\
\hline $94-124-01$ & 242-A & $242-A$ & 12.2 & 0.2 & $<0.5$ & 45 & $<1.6$ & $<50$ & $1,2,3,8$ \\
\hline $94-279-04$ & 242-A & 242-A & 178 & 0.2 & $<0.5$ & & & & 7 \\
\hline $94-279-05$ & $242-A$ & $242-A$ & 222 & 0.2 & $<0.5$ & & & & 7 \\
\hline
\end{tabular}


TANK FARMS LLW

93/94 HISTORICAL PIN FILES

\begin{tabular}{cccccccccc}
\hline PIN \# & Accum. Pt & $\begin{array}{c}\text { Point of } \\
\text { Origin }\end{array}$ & Weight & Volume & $6 \mathrm{pt}$ & ASSAY & SAMPLE & MAJOR CONTENTS \\
\hline & & & $(\mathrm{Kg})$ & $\left(\mathrm{m}^{3}\right)$ & $(\mathrm{mr} / \mathrm{hr})$ & $\begin{array}{c}\text { Cs-137 } \\
(\mathrm{pCi} / \mathrm{g})\end{array}$ & $\begin{array}{c}\text { Co-60 } \\
\text { (pCi/g) }\end{array}$ & \\
& & & & & & & & & \\
$93-118-03$ & $204-A R$ & $204-A R$ & 269 & 4.0 & $<0.5$ & & & $1,2,3,4$ \\
$93-153-02$ & 204-AR & 204-AR & 208 & 4.0 & $<0.5$ & & $1,2,3,4$ \\
$94-082-03$ & 204-AR & 204-AR & 419 & 4.0 & $<0.5$ & & & $1,2,3,4,5$
\end{tabular}

Contents Legend

1-Cloth, 2-Paper, 3-Plastic, 4-Rubber, 5-Metal, 6-Wood, 7-Concrete/Asphalt, 8-Vegetation, 9-Absorbent 
TANK FARMS LLW

93/94 HISTORICAL PIN FILES

\begin{tabular}{cccccccccc}
\hline PIN \# & Accum. Pt & $\begin{array}{c}\text { Point of } \\
\text { Origin }\end{array}$ & Weight & Volume & $6 \mathrm{pt}$ & ASSAY & SAMPLE & MAJOR CONTENTS \\
\hline & & $(\mathrm{Kg})$ & $\left(\mathrm{m}^{3}\right)$ & $(\mathrm{mr} / \mathrm{hr})$ & $\begin{array}{c}\text { Cs-137 } \\
(\mathrm{pCi} / \mathrm{g})\end{array}$ & $\begin{array}{c}\text { Co-60 } \\
\text { (pCi/g) }\end{array}$ & (see below) \\
$94-018-02$ & $209-\mathrm{E}$ & $209-\mathrm{E}$ & 34.5 & 0.2 & $<0.2$ & 5.9 & 9.80 & 11.10 & $1,2,9$
\end{tabular}


TANK FARMS LLW

93/94 HISTORICAL PIN FILES

\begin{tabular}{|c|c|c|c|c|c|c|c|c|c|}
\hline \multirow[t]{2}{*}{ PIN\# } & \multirow[t]{2}{*}{ Accum. $\overline{\mathrm{Pt}}$} & \multirow{2}{*}{$\begin{array}{l}\text { Point of } \\
\text { Origin }\end{array}$} & \multirow{2}{*}{$\begin{array}{c}\text { Weight } \\
(\mathrm{Kg})\end{array}$} & \multirow{2}{*}{$\frac{\text { Volume }}{\left(\mathrm{m}^{3}\right)}$} & \multirow{2}{*}{$\begin{array}{c}6 \mathrm{pt} \\
(\mathrm{mr} / \mathrm{hr})\end{array}$} & \multicolumn{2}{|c|}{ ASSAY } & \multirow{2}{*}{$\begin{array}{c}\text { SAMPLE } \\
\text { Tot. Act. } \\
\text { (pCi/g) }\end{array}$} & MAJOR CONTENTS \\
\hline & & & & & & $\begin{array}{r}\mathrm{Cs}-137 \\
\quad(\mathrm{pC}\end{array}$ & go-60 & & (see below) \\
\hline $93-039-03$ & s & sY & 580 & 4.0 & $<0.2$ & 380 & $<0.19$ & & $2,4,5,6$ \\
\hline $93-095-07$ & s & sy & 191 & 4.0 & & 350 & 1.1 & & $1,2,3,4$ \\
\hline $93-095-08$ & s & sY & 210 & 4.0 & & 3800 & 0.17 & & Eu-154: $22 \quad 1,2,3,4$ \\
\hline $93-095-09$ & s & SY & 224 & 4.0 & $<0.2$ & 32 & 1 & & $1,2,3,4,5,6,9$ \\
\hline $93-095-10$ & $\mathrm{~s}$ & SY & 633 & 4.0 & & & & & $1,2,3,4$ \\
\hline 93-103-01 & $\mathbf{s}$ & $\mathrm{S}$ & 646 & 4.0 & $<0.2$ & 30 & $<0.14$ & & $1,2,3,4,5,6,9$ \\
\hline $93-103-02$ & $\mathrm{~s}$ & $\mathbf{s}$ & 283 & 4.0 & & 0.57 & 2.1 & & $1,2,3,4$ \\
\hline 93-104-04 & $\mathrm{s}$ & $\mathbf{s}$ & 757 & 4.0 & $<0.2$ & 23 & $<0.16$ & & $3,4,5,6$ \\
\hline $93-107-01$ & $\mathrm{~s}$ & $\mathrm{~s}$ & 301 & 4.0 & & 40 & $<0.41$ & & $1,2,3,4$ \\
\hline $93-107-02$ & s & $\mathrm{s}$ & 227 & 4.0 & & & & & $1,2,3$ \\
\hline $93-107-03$ & s & $S$ & 865 & 4.0 & $<0.2$ & 25 & $<0.17$ & & $1,2,3,5,6,9$ \\
\hline $93-107-04$ & S & $S$ & 604 & 4.0 & $<0.2$ & 13 & $<0.27$ & & $1,3,4,5,9$ \\
\hline $93-107-06$ & $S$ & $s$ & 422 & 4.0 & $<0.2$ & 120 & $<0.27$ & & $1,2,3,5,6,9$ \\
\hline $93-109-13$ & $\mathrm{~s}$ & $242-S$ & 24 & 0.2 & $<0.5$ & 24 & $<18$ & $<50$ & $1,5,9$, OIL \\
\hline $93-110-12$ & $s$ & $s$ & 825 & 4.0 & $<0.2$ & 0.18 & 0.47 & & $1,2,3,5,6,9$ \\
\hline $93-110-14$ & $S$ & $s$ & 26 & 0.2 & $<0.5$ & 6.3 & $<1.7$ & $2.93 \mathrm{E}-05$ & 1,9, OIL \\
\hline $93-110-31$ & $S$ & SY & 249 & 4.0 & $<0.2$ & 0.88 & $<0.52$ & & $1,2,3,5,6,9$ \\
\hline $93-110-39$ & $S$ & $S$ & 538 & 4.0 & $<0.2$ & 270 & $<0.21$ & & $1,2,3,4,5,6,9$ \\
\hline $93-110-40$ & $S$ & S & 223 & 4.0 & $<0.2$ & 10 & 0.46 & & $3,8,9$ \\
\hline $93-110-41$ & $S$ & s & 286 & 4.0 & $<0.2$ & 100 & 3.5 & & $1,2,3,4,9$ \\
\hline $93-119-02$ & $\mathrm{~s}$ & $\mathrm{~s}$ & 310 & 4.0 & & & & & $1,2,3,4,9$ \\
\hline $93-119-03$ & $S$ & $S$ & 787 & 4.0 & 1 & 3500 & 0.83 & & $1,3,4,5,6,9$ \\
\hline & & & & & & Eu-154: 2 & Eu-155: & 3000 & \\
\hline
\end{tabular}


TANK FARMS LLW

93/94 HISTORICAL PIN FILES

\begin{tabular}{|c|c|c|c|c|c|c|c|c|c|}
\hline \multirow[t]{2}{*}{ PIN \# } & \multirow[t]{2}{*}{ Accum. Pt } & \multirow[t]{2}{*}{$\begin{array}{c}\text { Point of } \\
\text { Origin }\end{array}$} & \multirow{2}{*}{$\frac{\text { Weight }}{(\mathrm{Kg})}$} & \multirow{2}{*}{$\begin{array}{l}\text { Volume } \\
\left(\mathrm{m}^{3}\right)\end{array}$} & \multirow{2}{*}{$\frac{6 \mathrm{pt}}{(\mathrm{mr} / \mathrm{hr})}$} & \multicolumn{2}{|c|}{ ASSAY } & \multirow{2}{*}{$\begin{array}{l}\text { SAMPLE } \\
\text { Tot. Act. } \\
\text { (pCi/g) }\end{array}$} & \multirow{2}{*}{$\begin{array}{l}\text { MAJOR CONTENTS } \\
\text { (see below) }\end{array}$} \\
\hline & & & & & & $\begin{array}{r}\text { Cs-137 } \\
\text { (pC }\end{array}$ & Co-60 & & \\
\hline $93-138-02$ & $\mathrm{~s}$ & $\mathrm{~s}$ & 302 & 4.0 & $<0.2$ & 300 & 2.1 & & $1,2,3,4,9$ \\
\hline $93-160-02$ & $\mathrm{~s}$ & SISXISY & 535 & 4.0 & $<0.5$ & & & & $1,2,3,4,5,6,9$ \\
\hline $93-165-02$ & $S$ & SY & 216 & 4.0 & $<0.2$ & & & & $1,2,3,4,9$ \\
\hline $93-179-03$ & $\mathbf{S}$ & SY & 192 & 4.0 & $<0.2$ & & & & $1,2,3,4,9$ \\
\hline $93-179-04$ & $\mathrm{~S}$ & SY & 585 & 4.0 & $<0.2$ & & & & $1,2,3,4,5,6,9$ \\
\hline $93-181-02$ & $\mathrm{~S}$ & S-COMP. & 377 & 4.0 & $<0.2$ & & & & $1,2,3,4,9$ \\
\hline 93-195-03 & $\mathrm{s}$ & S-COMP. & 363 & 4.0 & $<0.2$ & & & & $1,2,3,4,9$ \\
\hline $93-267-01$ & $\mathrm{~S}$ & $\mathrm{~S}$ & 239 & 4.0 & $<0.2$ & & & & $1,2,3,4,9$ \\
\hline $93-312-02$ & S & S-COMP. & 371 & 4.0 & $<0.5$ & & & & $1,2,3,4,5,8,9$ \\
\hline $93-319-01$ & $S$ & SISXISY & 321 & 4.0 & $<0.2$ & & & & $1,2,3,4,5,6,9$ \\
\hline $93-321-02$ & $S$ & SXISY & 352 & 4.0 & $<0.2$ & & & & $1,2,3,4,5,9$ \\
\hline $93-363-02$ & S & S-COMP. & 273 & 4.0 & $<0.2$ & 810 & 3.1 & & $1,2,3,4,9$ \\
\hline $94-003-01$ & $S$ & $S$ & 1045 & 4.0 & $<0.5$ & & & & 6,9 \\
\hline $94-003-02$ & $S$ & $\mathrm{~s}$ & 975 & 4.0 & $<0.5$ & & & & 6,9 \\
\hline $94-066-01$ & $S$ & S-COMP. & 969 & 4.0 & $<0.5$ & & & & $3,4,5,6,9$ \\
\hline $94-082-02$ & s & SY & 344 & 4.0 & $<0.5$ & & & & $1,2,3,4,5,6,9$ \\
\hline $94-109-01$ & S & S-COMP. & 369 & 4.0 & $<0.5$ & 330 & 2.1 & & $1,2,3,4,5,6,9$ \\
\hline $94-139-03$ & $S$ & S-COMP. & 450 & 4.0 & $<0.5$ & & & & $1,2,3,4,5,6,8,9$ \\
\hline $94-168-01$ & S & S-COMP. & 492 & 4.0 & $<0.5$ & & & & $1,2,3,4,5,6,9$ \\
\hline
\end{tabular}


TANK FARMS LLW

93/94 HISTORICAL PIN FILES

\begin{tabular}{|c|c|c|c|c|c|c|c|c|}
\hline PIN \# & Accum. $\overline{\mathbf{P t}}$ & $\begin{array}{l}\text { Point of } \\
\text { Origin }\end{array}$ & Weight & Volume & $6 \mathrm{pt}$ & ASSAY & SAMPLE & MAJOR CONTENTS \\
\hline & & & $(\mathrm{Kg})$ & $\left(\mathrm{m}^{3}\right)$ & $(\mathrm{mr} / \mathrm{hr})$ & $\begin{array}{c}\text { Cs-137 Co-60 } \\
\text { (pCi/g) }\end{array}$ & $\begin{array}{l}\text { Tot. Act. } \\
\text { (pCi/g) }\end{array}$ & (see below) \\
\hline $94-171-01$ & $S$ & SY & 351 & 4.0 & $<0.5$ & & & $1,2,3,4,5,6,9$ \\
\hline $94-227-01$ & $\mathrm{~S}$ & S-COMP. & 260 & 4.0 & $<0.5$ & & & $1,2,3,4,5,6,9$ \\
\hline $94-243-01$ & $\mathrm{~s}$ & SY & 623 & 4.0 & $<0.5$ & & & $5,6,9$ \\
\hline $94-243-02$ & $S$ & SY & 564 & 4.0 & $<0.5$ & & & $5,6,9$ \\
\hline $94-244-02$ & $S$ & SY & 855 & 4.0 & $<0.5$ & & & $2,3,5,6$ \\
\hline $94-244-03$ & $S$ & SY & 21 & 0.2 & $<0.5$ & & & $1,3,6,9$, OIL \\
\hline $94-244-04$ & $S$ & SY & 26 & 0.2 & $<0.5$ & & & $1,2,3,5,9$, OIL \\
\hline $94-244-04$ & $\mathrm{~S}$ & SY & 26 & 0.2 & $<0.5$ & & & $1,2,3,5,9$, OIL \\
\hline $94-249-01$ & $\mathrm{~s}$ & SY & 296 & 4.0 & $<0.5$ & & & $1,2,3,4,5,6,9$ \\
\hline $94-249-02$ & $\mathrm{~s}$ & S-COMP. & 368 & 4.0 & $<0.5$ & & & $1,2,3,4,5,6,9$ \\
\hline $94-250-01$ & $\mathrm{~s}$ & $\mathrm{~s}$ & 619 & 4.0 & $<0.5$ & & & $1,2,3,4,5,6,9$ \\
\hline $94-269-01$ & $\mathrm{~S}$ & SY & 269 & 4.0 & $<0.5$ & & & $1,2,3,4,5,6,9$ \\
\hline $94-277-01$ & $\mathrm{~s}$ & S-COMP. & 405 & 4.0 & $<0.5$ & & & $1,2,3,4,5,6,9$ \\
\hline
\end{tabular}

Contents Legend:

1-Cloth, 2-Paper, 3-Plastic, 4-Rubber, 5-Metal, 6-Wood, 7-Concrete/Asphalt, 8-Vegetation, 9-Absorbent 
TANK FARMS LIW

93/94 HISTORICAL PIN FILES

\begin{tabular}{|c|c|c|c|c|c|c|c|c|c|}
\hline \multirow[t]{2}{*}{ PIN \# } & \multirow[t]{2}{*}{ Accum. Pt } & \multirow{2}{*}{$\begin{array}{c}\text { Point of } \\
\text { Origin }\end{array}$} & \multirow{2}{*}{$\frac{\text { Weight }}{(\mathrm{Kg})}$} & \multirow{2}{*}{$\frac{\text { Volume }}{\left(\mathrm{m}^{3}\right)}$} & \multirow{2}{*}{$\begin{array}{c}6 \mathrm{pt} \\
(\mathrm{mr} / \mathrm{hr})\end{array}$} & \multicolumn{2}{|c|}{ ASSAY } & \multirow{2}{*}{$\begin{array}{c}\text { SAMPLE } \\
\text { Tot. Act. } \\
\text { (pCi/g) }\end{array}$} & \multirow{2}{*}{$\begin{array}{c}\text { MAJOR CONTENTS } \\
\text { (see below) }\end{array}$} \\
\hline & & & & & & $\begin{array}{r}\text { Cs-137 } \\
(\mathrm{p}\end{array}$ & $\begin{array}{l}\text { Co-60 } \\
\text { g) }\end{array}$ & & \\
\hline 93-090-01 & $s x$ & 701 Bldg & 1646 & 4.0 & & 0.29 & $<0.23$ & & $3,6,7$ \\
\hline 93-090-03 & $s x$ & 701 Bidg & 1744 & 4.0 & $<0.2$ & 0.26 & $<0.19$ & & 5 \\
\hline $93-104-06$ & $s x$ & sx & 1535 & 4.0 & $<0.2$ & 0.25 & $<0.18$ & & $1,2,3,4,5$ \\
\hline $93-104-09$ & $s x$ & 701 Bldg & 1694 & 4.0 & $<0.2$ & 0.31 & $<0.21$ & & $5,6,7$ \\
\hline $93-110-27$ & $s x$ & sx & 1048 & 4.0 & $<0.2$ & 0.25 & $<0.19$ & & 5 \\
\hline $93-110-28$ & $s x$ & $\mathrm{sx}$ & 440 & 4.0 & $<0.2$ & 0.36 & $<0.25$ & & 5,6 \\
\hline $93-110-29$ & $s x$ & $\mathrm{sX}$ & 383 & 4.0 & $<0.2$ & 0.34 & $<0.32$ & & 5,9 \\
\hline $93-119-01$ & $s x$ & S/SX/SY & 302 & 4.0 & & & & & $1,2,3,4,5,9$ \\
\hline $93-110-30$ & $s x$ & $s x$ & 511 & 4.0 & & 0.25 & 0.19 & & $3,5,6,9$ \\
\hline $93-363-01$ & $s x$ & S/SX & 569 & 4.0 & 0.8 & 2700 & 5.3 & & $1,2,3,4,5,9$ \\
\hline $94-031-02$ & $s x$ & S-COMP. & 279 & 4.0 & $<0.5$ & 610 & 10.00 & & $1,2,3,4,9$ \\
\hline 94-088-01 & $s x$ & S-COMP. & 627 & 4.0 & $<0.5$ & 57 & 1.90 & & $1,2,3,4,5,9$ \\
\hline
\end{tabular}


TANK FARMS LLW

93/94 HISTORICAL PIN FILES

\begin{tabular}{|c|c|c|c|c|c|c|c|c|c|}
\hline \multirow[t]{2}{*}{ PIN \# } & \multirow[t]{2}{*}{ Accum. Pt } & \multirow{2}{*}{$\begin{array}{l}\text { Point of } \\
\text { Origin }\end{array}$} & \multirow{2}{*}{$\frac{\text { Weight }}{(\mathrm{Kg})}$} & \multirow{2}{*}{$\frac{\text { Volume }}{\left(\mathrm{m}^{3}\right)}$} & \multirow{2}{*}{$\begin{array}{c}6 \mathrm{pt} \\
(\mathrm{m} / \mathrm{hr})\end{array}$} & \multicolumn{2}{|c|}{ ASSAY } & \multirow{2}{*}{$\begin{array}{c}\text { SAMPLE } \\
\begin{array}{c}\text { Tot. Act. } \\
\text { (pCi/g) }\end{array}\end{array}$} & \multirow{2}{*}{$\begin{array}{c}\text { MAJOR CONTENTS } \\
\text { (see below) }\end{array}$} \\
\hline & & & & & & Cs-137 & $\begin{array}{l}\text { Co-60 } \\
\text { g) }\end{array}$ & & \\
\hline $93-103-07$ & $T$ & $T$ & 204 & 4.0 & $<0.2$ & 11 & 2.30 & & $1,2,3,4,8,9$ \\
\hline $93-103-08$ & $T$ & $T$ & 355 & 4.0 & $<0.5$ & 780 & 0.70 & & $1,2,4,5,9$ \\
\hline $93-110-21$ & $T$ & $T$ & 39 & 4.0 & $<0.2$ & & & & 8 \\
\hline $93-160-01$ & $T$ & $T$ & 312 & 4.0 & $<0.2$ & 10 & 5.40 & & $1,2,3,4,9$ \\
\hline $93-165-03$ & $\mathbf{T}$ & T & 255 & 4.0 & $<0.5$ & 40 & 4.30 & & $1,2,3,4,9$ \\
\hline $94-103-01$ & $\mathbf{T}$ & $T$ & 551 & 4.0 & $<0.2$ & 95 & 0.47 & & $1,2,3,4,5,9$ \\
\hline $94-125-02$ & $T$ & $T$ & 473 & 4.0 & $<0.5$ & & & & $1,2,3,4,5,6,9$ \\
\hline $94-130-05$ & $T$ & $T$ & 587 & 4.0 & $<0.5$ & 0.6 & $<0.28$ & & 6 \\
\hline
\end{tabular}


TANK FARMS LLW

93/94 HISTORICAL PIN FILES

\begin{tabular}{|c|c|c|c|c|c|c|c|c|c|}
\hline \multirow[t]{2}{*}{ PIN \# } & \multirow[t]{2}{*}{ Accum. Pt } & \multirow{2}{*}{$\begin{array}{c}\text { Point of } \\
\text { Origin }\end{array}$} & \multirow{2}{*}{$\begin{array}{c}\text { Weight } \\
(\mathrm{Kg})\end{array}$} & \multirow{2}{*}{$\frac{\text { Volume }}{\left(\mathrm{m}^{3}\right)}$} & \multirow{2}{*}{$\frac{6 \mathrm{pt}}{(\mathrm{mr} / \mathrm{hr})}$} & \multicolumn{2}{|c|}{ ASSAY } & \multirow{2}{*}{$\begin{array}{l}\text { SAMPLE } \\
\text { Tot. Act. } \\
(\mathrm{pCi} / \mathrm{g})\end{array}$} & \multirow{2}{*}{$\begin{array}{l}\text { MAJOR CONTENTS } \\
\text { (see below) }\end{array}$} \\
\hline & & & & & & $\begin{array}{r}\text { Cs-137 } \\
\text { (p }\end{array}$ & $\begin{array}{l}\text { Co-60 } \\
\text { g) }\end{array}$ & & \\
\hline $93-103-05$ & TX & $T X$ & 632 & 4.0 & $<0.2$ & 1.5 & $<.17$ & & $2,5,6,9$ \\
\hline $93-103-06$ & $T X$ & TX & 243 & 4.0 & $<0.2$ & 30.0 & 3.70 & & $1,2,3,4,8,9$ \\
\hline $93-107-07$ & $\mathrm{TX}$ & TX & 245 & 4.0 & & & & & $1,2,3,4,8,9$ \\
\hline $93-107-08$ & TX & TX & 799 & 4.0 & $<0.5$ & & & & $1,3,4,5,6$ \\
\hline $93-126-03$ & TX & TX & 376 & 4.0 & $<0.2$ & & & & $1,2,3,4,9$ \\
\hline $93-133-01$ & $\mathrm{TX}$ & TX & 95 & 0.2 & $<0.5$ & & & & $1,5,7$ \\
\hline $93-195-01$ & TX & TX,TY & 836 & 4.0 & $<0.2$ & & & & $5,6,9$ \\
\hline 93-195-01 & TX & TX & 744 & 4.0 & $<0.2$ & & & & 6,9 \\
\hline $93-211-03$ & TX & $T X$ & 919 & 4.0 & $<0.2$ & & & & $5,6,9$ \\
\hline $93-364-01$ & $\mathrm{TX}$ & $T X$ & 465 & 4.0 & $<0.5$ & & & & $1,2,3,4,9$ \\
\hline $94-152-02$ & $\mathrm{TX}$ & TX,TY & 641 & 4.0 & $<0.5$ & 220.0 & $<0.24$ & & $5,6,7,9$ \\
\hline $94-206-03$ & $\mathrm{TX}$ & TX & 292 & 4.0 & $<0.5$ & & & & $1,2,3,4,5,8,9$ \\
\hline
\end{tabular}


TANK FARMS LLW

93/94 HISTORICAL PIN FILES

\begin{tabular}{|c|c|c|c|c|c|c|c|c|c|}
\hline \multirow[t]{2}{*}{$\mathrm{PIN} \#$} & \multirow[t]{2}{*}{ Accum $\mathbf{P t}$} & \multirow{2}{*}{$\begin{array}{c}\text { Point of } \\
\text { Origin }\end{array}$} & \multirow{2}{*}{$\begin{array}{c}\text { Weight } \\
(\mathrm{Kg})\end{array}$} & \multirow{2}{*}{$\begin{array}{l}\text { Volume } \\
\left(\mathrm{m}^{3}\right)\end{array}$} & \multirow{2}{*}{$\begin{array}{c}6 \mathrm{pt} \\
\frac{(\mathrm{mr} / \mathrm{hr})}{}\end{array}$} & \multicolumn{2}{|c|}{ ASSAY } & \multirow{2}{*}{$\begin{array}{c}\text { SAMPLE } \\
\text { Tot. Act. } \\
\text { (pCi/g) }\end{array}$} & \multirow{2}{*}{$\begin{array}{c}\text { MAJOR CONTENTS } \\
\text { (see below) }\end{array}$} \\
\hline & & & & & & $\begin{array}{r}\text { Cs-137 } \\
(p\end{array}$ & $\begin{array}{l}\mathrm{Co-60} \\
\text { g) }\end{array}$ & & \\
\hline $93-103-03$ & $\mathrm{U}$ & $U$ & 730 & 4.0 & $<0.5$ & & & & $1,2,3,4,5,6,9$ \\
\hline $93-103-04$ & $\mathrm{U}$ & $u$ & 263 & 4.0 & $<0.2$ & 4.1 & 0.38 & & $1,2,3,4$ \\
\hline $93-110-13$ & $\mathrm{U}$ & $152-U \mathrm{DB}$ & 236 & 4.0 & & 0.86 & $<0.53$ & & $2,3,4,5,6,7$ \\
\hline $93-327-01$ & U & U & 269 & 4.0 & $<0.5$ & & & & $1,2,3,4,9$ \\
\hline $93-327-01$ & $\mathrm{u}$ & $\mathbf{u}$ & 422 & 4.0 & $<0.5$ & & & & $1,2,3,4,5,6,9$ \\
\hline 94-206-02 & $\mathrm{U}$ & $\mathbf{U}$ & 301 & 4.0 & $<0.5$ & & & & $1,2,3,4,5,6,9$ \\
\hline $94-227-01$ & $\mathrm{U}$ & U & 346 & 4.0 & $<0.5$ & & & & $1,2,3,4,5,6,9$ \\
\hline
\end{tabular}


TANK FARMS LLW

93/94 HISTORICAL PIN FILES

\begin{tabular}{|c|c|c|c|c|c|c|c|c|c|}
\hline \multirow[t]{2}{*}{ PIN \# } & \multirow[t]{2}{*}{ Accum. Pt } & \multirow{2}{*}{$\begin{array}{l}\text { Point of } \\
\text { Origin }\end{array}$} & \multirow{2}{*}{$\frac{\text { Weight }}{(\mathrm{Kg})}$} & \multirow{2}{*}{$\frac{\text { Volume }}{\left(\mathrm{m}^{3}\right)}$} & \multirow{2}{*}{$\begin{array}{c}6 \mathrm{pt} \\
(\mathrm{mr} / \mathrm{hr})\end{array}$} & \multicolumn{2}{|c|}{ ASSAY } & \multirow{2}{*}{$\begin{array}{c}\text { SAMPLE } \\
\text { Tot. Act. } \\
(\mathrm{pCi} / \mathrm{g})\end{array}$} & \multirow{2}{*}{$\begin{array}{l}\text { MAJOR CONTENTS } \\
\text { (see below) }\end{array}$} \\
\hline & & & & & & $\begin{array}{r}\text { Cs-137 } \\
\text { (p }\end{array}$ & $\begin{array}{l}\text { Co-60 } \\
\text { g) }\end{array}$ & & \\
\hline 93-153-01 & 2713-WB & 2713-WB & 37.2 & 0.2 & $<0.5$ & 4.4 & $<2.4$ & & 1, 9, OIL \\
\hline $94-053-01$ & 2713-WB & 2713-WB & 903 & 4.0 & $<0.5$ & & & & $2,3,5,6,9$ \\
\hline $94-053-02$ & 2713-WB & 2713-WB & 1988 & 4.0 & $<0.5$ & & & $<50$ & $3,4,5,6,9$ \\
\hline $94-053-03$ & 2713-WB & 2713-WB & 767 & 4.0 & $<0.5$ & & & & $1,3,4,5,6,9$ \\
\hline $94-053-04$ & 2713-WB & 2713-WB & 1721 & 4.0 & $<0.5$ & & & & $2,4,5,6,9$ \\
\hline $94-059-01$ & 2713-WB & 2713-WB & 28.6 & 0.2 & $<0.5$ & 1.5 & $<1.9$ & 178 & $2,5,9$, OIL \\
\hline $94-059-02$ & 2713-WB & 2713-WB & 47.6 & 0.2 & $<0.5$ & 3.1 & 3.5 & $<50$ & $1,9, \mathrm{OL}$ \\
\hline $94-059-03$ & 2713-WB & 2713-WB & 162 & 0.2 & $<0.5$ & 5.4 & $<0.93$ & 990 & 1,9, OIL \\
\hline $94-067-04$ & 2713-WB & 2713-WB & 1975 & 4.0 & $<0.5$ & & & & 5,9 \\
\hline $94-069-03$ & 2713-WB & 2713-WB & 1542 & 4.0 & $<0.5$ & & & & 4,5 \\
\hline $94-074-01$ & 2713-WB & 2713-WB & 1011 & 4.0 & $<0.5$ & $<0.32$ & $<0.26$ & & $3,5,6,9$ \\
\hline $94-087-03$ & 2713-WB & 2713-WB & 642 & 4.0 & $<0.5$ & $<0.45$ & $<0.33$ & & $3,4,5,9$ \\
\hline $94-115-01$ & 2713-WB & 2713-WB & 583 & 4.0 & $<0.5$ & 1.8 & $<0.4$ & 7.12E-03 & $3,5,6$ \\
\hline $94-117-01$ & 2713-WB & 2713-WB & 297 & 4.0 & $<0.5$ & 2.8 & $<0.85$ & & 6,9 \\
\hline
\end{tabular}


WHC-SD-WM-ER-435, Rev. 1

Page B-i

ATTACHMENT B 


\section{Low Level Waste Sample. Set \#1}

pCi/Sample (1)

\begin{tabular}{lccccccc} 
Farm/Area & Total Beta & Total Alpha & Cs-137 & Co-60 & Eu-152 & Eu-154 & Eu-155 \\
\hline \hline & & & & & & & \\
241-A & $6.84 \mathrm{E}+02$ & $7.12 \mathrm{E}+00$ & $6.72 \mathrm{E}+02$ & $7.20 \mathrm{E}+01$ & & & \\
$241-\mathrm{AN}$ & $1.72 \mathrm{E}+03$ & $<6.53 \mathrm{E}+1$ & $5.66 \mathrm{E}+02$ & & & & \\
$241-\mathrm{AP}$ & $3.94 \mathrm{E}+03$ & $8.14 \mathrm{E}+02$ & $1.97 \mathrm{E}+03$ & $5.61 \mathrm{E}+02$ & & $3.43 \mathrm{E}+02$ & \\
$241-\mathrm{AW}$ & $3.25 \mathrm{E}+03$ & $2.75 \mathrm{E}+01$ & $5.16 \mathrm{E}+03$ & $1.22 \mathrm{E}+03$ & & \\
$241-\mathrm{AY}$ & $4.15 \mathrm{E}+03$ & $1.84 \mathrm{E}+01$ & $1.48 \mathrm{E}+03$ & $7.57 \mathrm{E}+01$ & & & \\
$244-\mathrm{A}$ & $7.56 \mathrm{E}+02$ & $2.50 \mathrm{E}+01$ & $6.21 \mathrm{E}+02$ & $2.47 \mathrm{E}+02$ & & \\
$242-\mathrm{A}$ & $2.78 \mathrm{E}+03$ & $2.48 \mathrm{E}+02$ & $1.60 \mathrm{E}+03$ & $7.64 \mathrm{E}+02$ & & & \\
204-AR & $2.55 \mathrm{E}+03$ & $8.77 \mathrm{E}+01$ & $1.94 \mathrm{E}+03$ & $5.40 \mathrm{E}+01$ & $5.98 \mathrm{E}+02$ & $8.49 \mathrm{E}+02$ & $3.28 \mathrm{E}+02$ \\
$241-\mathrm{B}$ & $2.24 \mathrm{E}+03$ & $2.24 \mathrm{E}+01$ & $5.92 \mathrm{E}+02$ & $5.13 \mathrm{E}+03$ & & & \\
$241-\mathrm{BX} / \mathrm{BY}$ & $2.76 \mathrm{E}+03$ & $3.39 \mathrm{E}+01$ & $2.34 \mathrm{E}+03$ & $4.90 \mathrm{E}+02$ & & & \\
$241-\mathrm{C}$ & $3.48 \mathrm{E}+04$ & $<1.96 \mathrm{E}+2$ & $2.83 \mathrm{E}+03$ & $1.43 \mathrm{E}+02$ & & & \\
209-E & $6.10 \mathrm{E}+02$ & $1.42 \mathrm{E}+02$ & $1.88 \mathrm{E}+02$ & $1.74 \mathrm{E}+02$ & & & \\
$241-\mathrm{S} / \mathrm{SX}$ & $2.10 \mathrm{E}+03$ & $<3.22 \mathrm{E}+2$ & $9.59 \mathrm{E}+02$ & $1.67 \mathrm{E}+03$ & & & \\
$241-\mathrm{SY}$ & $<1.31 \mathrm{E}+3$ & $<4.93 \mathrm{E}+2$ & $4.79 \mathrm{E}+02$ & & & & \\
$241-\mathrm{T}$ & $4.18 \mathrm{E}+03$ & $3.96 \mathrm{E}+02$ & $1.46 \mathrm{E}+03$ & $3.10 \mathrm{E}+02$ & & & \\
$241-\mathrm{TX} / \mathrm{TY}$ & $5.25 \mathrm{E}+02$ & $1.24 \mathrm{E}+02$ & $2.24 \mathrm{E}+02$ & & & & \\
$241-\mathrm{U}$ & $4.92 \mathrm{E}+02$ & $<2.85 \mathrm{E}+2$ & $2.86 \mathrm{E}+02$ & $1.10 \mathrm{E}+02$ & & & \\
& & & & & & & \\
Average & $4.22 \mathrm{E}+03$ & $1.62 \mathrm{E}+02$ & $1.37 \mathrm{E}+03$ & $7.87 \mathrm{E}+02$ & $5.98 \mathrm{E}+02$ & $5.96 \mathrm{E}+02$ & $3.28 \mathrm{E}+02$ \\
Std. Dev. & $8.26 \mathrm{E}+03$ & $2.36 \mathrm{E}+02$ & $1.26 \mathrm{E}+03$ & $1.34 \mathrm{E}+03$ & & & \\
95\% Conf. & $4.05 \mathrm{E}+03$ & $1.33 \mathrm{E}+02$ & $5.98 \mathrm{E}+02$ & $7.01 \mathrm{E}+02$ & & &
\end{tabular}

(1) A blank indicates no activity detected

\section{NOTE THAT THESE SAMPLE RESULTS ARE DCISAMPLE NOT PER GRAM}




\section{Low Level Waste Sample Set \#2}

pCi/g (1)

\begin{tabular}{lcccc} 
Farm/Area & Total Beta & Total Alpha & Cs-137 & Co-60 \\
\hline \hline & & & & \\
$241-\mathrm{A}$ & $2.92 \mathrm{E}+00$ & $2.19 \mathrm{E}-01$ & $2.21 \mathrm{E}+00$ & $2.94 \mathrm{E}-01$ \\
$241-\mathrm{AN}$ & $1.28 \mathrm{E}+01$ & $1.70 \mathrm{E}+00$ & $1.80 \mathrm{E}+01$ & \\
$241-\mathrm{AP}$ & $1.19 \mathrm{E}+01$ & $6.28 \mathrm{E}-01$ & $1.29 \mathrm{E}+01$ & $1.01 \mathrm{E}+00$ \\
$241-\mathrm{AW}$ & $2.53 \mathrm{E}+01$ & $1.64 \mathrm{E}+00$ & $1.20 \mathrm{E}+01$ & $7.86 \mathrm{E}-01$ \\
$241-\mathrm{AY}$ & $9.85 \mathrm{E}+00$ & $9.75 \mathrm{E}-01$ & $5.57 \mathrm{E}+00$ & $3.39 \mathrm{E}+00$ \\
$244-\mathrm{A}$ & $5.80 \mathrm{E}+00$ & $1.27 \mathrm{E}+00$ & $1.58 \mathrm{E}+00$ & $1.07 \mathrm{E}+01$ \\
$242-\mathrm{A}$ & $4.70 \mathrm{E}+00$ & $2.50 \mathrm{E}-01$ & $3.20 \mathrm{E}+00$ & $6.22 \mathrm{E}+00$ \\
$204-\mathrm{AR}$ & $2.56 \mathrm{E}+00$ & $4.52 \mathrm{E}-01$ & $1.02 \mathrm{E}+00$ & $7.67 \mathrm{E}-01$ \\
$241-\mathrm{B}$ & $1.80 \mathrm{E}+01$ & $1.94 \mathrm{E}-01$ & $7.43 \mathrm{E}+00$ & $2.52 \mathrm{E}+00$ \\
$241-\mathrm{BX} / \mathrm{BY}$ & $2.90 \mathrm{E}+01$ & $9.98 \mathrm{E}-02$ & $2.14 \mathrm{E}+01$ & $3.75 \mathrm{E}+00$ \\
$241-\mathrm{C}$ & $1.52 \mathrm{E}+01$ & $<9.95 \mathrm{E}-2$ & $7.90 \mathrm{E}+00$ & $1.66 \mathrm{E}+00$ \\
$209-\mathrm{E}$ & $3.12 \mathrm{E}+00$ & $<8.18 \mathrm{E}-2$ & $1.61 \mathrm{E}+00$ & $9.77 \mathrm{E}-01$ \\
$241-\mathrm{S} / \mathrm{SX}$ & $1.56 \mathrm{E}+01$ & $5.28 \mathrm{E}-01$ & $9.18 \mathrm{E}+00$ & $4.26 \mathrm{E}+01$ \\
$241-\mathrm{SY}$ & $3.04 \mathrm{E}+01$ & $1.08 \mathrm{E}+00$ & $2.81 \mathrm{E}+01$ & $6.06 \mathrm{E}-01$ \\
$241-\mathrm{T}$ & $7.34 \mathrm{E}+00$ & $<2.12 \mathrm{E}-1$ & $2.44 \mathrm{E}+00$ & $3.47 \mathrm{E}-01$ \\
$241-\mathrm{TX} / \mathrm{TY}$ & $7.10 \mathrm{E}+00$ & $<1.86 \mathrm{E}-1$ & $7.03 \mathrm{E}+00$ & \\
$241-\mathrm{U}$ & $1.53 \mathrm{E}+01$ & $4.91 \mathrm{E}-01$ & $1.38 \mathrm{E}+01$ & $6.26 \mathrm{E}+00$ \\
& & & & \\
Average & $1.28 \mathrm{E}+01$ & $7.33 \mathrm{E}-01$ & $9.14 \mathrm{E}+00$ & $5.46 \mathrm{E}+00$ \\
Std. Dev. & $8.87 \mathrm{E}+00$ & $5.48 \mathrm{E}-01$ & $7.75 \mathrm{E}+00$ & $1.07 \mathrm{E}+01$ \\
95\% Conf. & $4.21 \mathrm{E}+00$ & $2.98 \mathrm{E}-01$ & $3.69 \mathrm{E}+00$ & $5.41 \mathrm{E}+00$
\end{tabular}

(1) A blank indicates no activity detected 


\section{Low Level Waste Sample Set \#3}

DCi/g (1)

\begin{tabular}{|c|c|c|c|c|c|c|}
\hline Farm/Area & Total Beta & Total Alpha & Cs- 137 & $\mathrm{Co}-60$ & $\mathrm{Sr}-90$ & Pu-239-240 \\
\hline $241-A$ & $2.90 \mathrm{E}-01$ & $<4.83 \mathrm{E}-2$ & $2.68 \mathrm{E}-01$ & & $<5.33 \mathrm{E}-1$ & $<2.04 \mathrm{E}-1$ \\
\hline $241-A N$ & $4.44 \mathrm{E}+02$ & $<7.01 \mathrm{E}-2$ & $5.88 \mathrm{E}-01$ & & & \\
\hline 241-AP & $7.93 \mathrm{E}+00$ & $4.18 \mathrm{E}-01$ & $1.73 E+00$ & $3.40 \mathrm{E}-01$ & & \\
\hline 241-AW & $9.70 \mathrm{E}+00$ & $<1.01 \mathrm{E}-1$ & $2.07 \mathrm{E}+00$ & $7.05 \mathrm{E}-01$ & & \\
\hline 241-AY & $1.01 \mathrm{E}+00$ & $<4.76 \mathrm{E}-2$ & $3.30 \mathrm{E}-01$ & & $8.48 \mathrm{E}-01$ & $<1.43 \mathrm{E}-1$ \\
\hline 244-A & $3.67 \mathrm{E}+00$ & $<7.47 \mathrm{E}-2$ & $6.09 \mathrm{E}-01$ & & $2.19 \mathrm{E}+00$ & $<3.33 \mathrm{E}-1$ \\
\hline $242-A$ & $1.47 \mathrm{E}+01$ & $1.71 \mathrm{E}-01$ & $4.20 \mathrm{E}+00$ & $9.97 \mathrm{E}-01$ & $2.12 \mathrm{E}+00$ & $<1.31 \mathrm{E}-1$ \\
\hline 204-AR & $2.86 \mathrm{E}+01$ & $7.25 \mathrm{E}-01$ & $7.77 \mathrm{E}+00$ & $2.53 \mathrm{E}+00$ & $1.15 \mathrm{E}+01$ & $2.11 \mathrm{E}-01$ \\
\hline $241-B$ & $5.14 \mathrm{E}+01$ & $4.64 \mathrm{E}-01$ & $1.12 \mathrm{E}+01$ & $1.16 \mathrm{E}+00$ & & \\
\hline 241-BX/BY & $2.04 \mathrm{E}+0 \mathrm{I}$ & $3.09 \mathrm{E}+00$ & $1.37 \mathrm{E}+01$ & $1.49 \mathrm{E}+00$ & $5.02 \mathrm{E}+00$ & $<3.05 \mathrm{E}-1$ \\
\hline $241-C$ & $2.88 \mathrm{E}+01$ & $<4.23 \mathrm{E}-2$ & $5.45 E+01$ & & $9.92 \mathrm{E}-01$ & $<1.12 \mathrm{E}-1$ \\
\hline $209-E$ & $4.88 \mathrm{E}+00$ & $1.23 \mathrm{E}-01$ & $2.84 \mathrm{E}+00$ & & & \\
\hline 241-S/SX & $1.07 \mathrm{E}+00$ & $<7.75 \mathrm{E}-2$ & $9.11 \mathrm{E}-01$ & & & \\
\hline 24l-SY & $4.29 \mathrm{E}+00$ & $<6.82 \mathrm{E}-2$ & $3.25 \mathrm{E}+00$ & & & \\
\hline $241-T$ & $1.98 E+00$ & $<7.84 \mathrm{E}-2$ & $1.41 E+00$ & & & \\
\hline 24l-TX/TY & $9.28 \mathrm{E}+00$ & $2.65 E-01$ & $6.19 \mathrm{E}+00$ & $4.41 \mathrm{E}-01$ & & \\
\hline $241-U$ & $4.40 \mathrm{E}+00$ & $2.03 \mathrm{E} \sim 01$ & $2.66 \mathrm{E}+00$ & $5.56 \mathrm{E}-01$ & & \\
\hline Average & $3.74 \mathrm{E}+01$ & $6.82 \mathrm{E}-01$ & $6.72 \mathrm{E}+00$ & $1.03 E+00$ & $3.78 \mathrm{E}+00$ & $2.11 \mathrm{E}-01$ \\
\hline Std. Dev. & $1.06 \mathrm{E}+02$ & $9.92 \mathrm{E}-01$ & $1.29 \mathrm{E}+01$ & $7.20 \mathrm{E}-01$ & $4.07 E+00$ & \\
\hline $95 \%$ Conf. & $5.02 \mathrm{E}+01$ & $6.88 \mathrm{E}-01$ & $6.14 \mathrm{E}+00$ & $4.99 \mathrm{E}-01$ & $3.26 \mathrm{E}+00$ & \\
\hline
\end{tabular}

(1) A blank indicates no activity detected 


\section{Low Level Waste Sample Set \#4}

\section{pCi/g (1)}

\begin{tabular}{|c|c|c|c|c|c|c|c|c|}
\hline Farm/Area & Total Beta & Total Alpha & Cs- 137 & $\mathrm{Co}-60$ & $\mathrm{Sr}-90$ & Tc-99 & Pu-239/240 & Total U (2) \\
\hline 241-AN & $6.14 \mathrm{E}+00$ & $<.731$ & $6.51 \mathrm{E}+00$ & $2.12 \mathrm{E}+00$ & $1.99 \mathrm{E}+00$ & $<35.8$ & $<2.88$ & $4.47 \mathrm{E}-09$ \\
\hline 241-AP & $1.99 E+01$ & $1.18 \mathrm{E}+00$ & $6.49 \mathrm{E}+00$ & $5.70 \mathrm{E}+00$ & $5.30 \mathrm{E}+00$ & $<32.9$ & $<0.66$ & 8.07E-09 \\
\hline 241-AW & $4.94 \mathrm{E}+00$ & $<.493$ & $4.83 \mathrm{E}+00$ & & $1.30 \mathrm{E}+00$ & $<41.8$ & $<2.38$ & $9.47 \mathrm{E}-08$ \\
\hline 241-AY & $1.88 \mathrm{E}+01$ & $5.29 \mathrm{E}-01$ & $7.36 \mathrm{E}+00$ & $7.83 \mathrm{E}-01$ & $6.74 \mathrm{E}+00$ & $<6.9$ & $<3.11$ & $1.04 \mathrm{E}-08$ \\
\hline $244-A$ & $8.53 \mathrm{E}+00$ & $3.74 \mathrm{E}-01$ & $3.25 \mathrm{E}+00$ & $1.10 \mathrm{E}+00$ & $4.38 \mathrm{E}+00$ & $<32.8$ & $<1.86$ & $6.77 \mathrm{E}-08$ \\
\hline $242-A$ & $1.93 \mathrm{E}+01$ & $7.99 \mathrm{E}-01$ & $9.65 \mathrm{E}+00$ & $1.09 \mathrm{E}+00$ & $4.96 \mathrm{E}+00$ & $<29.1$ & $<0.667$ & $2.35 \mathrm{E}-08$ \\
\hline 204-AR & $1.79 E+01$ & $3.92 \mathrm{E}-01$ & $7.19 \mathrm{E}+00$ & $2.62 \mathrm{E}+00$ & $7.90 \mathrm{E}+00$ & $<32.6$ & $<1.84$ & $3.63 \mathrm{E}-08$ \\
\hline 241-B & $2.29 \mathrm{E}+01$ & $<381$ & $9.45 \mathrm{E}+00$ & $2.30 \mathrm{E}+00$ & $6.51 \mathrm{E}+00$ & $<33.3$ & $<1.7$ & $1.87 \mathrm{E}-08$ \\
\hline 241-BX/BY & $1.36 \mathrm{E}+01$ & $1.21 \mathrm{E}+00$ & $8.61 \mathrm{E}+00$ & 9.97E-01 & $4.03 \mathrm{E}+00$ & $<38$ & $<1.16$ & $1.02 \mathrm{E}-08$ \\
\hline $241-\mathrm{C}$ & $7.46 \mathrm{E}+00$ & 8.37E-01 & $4.06 \mathrm{E}+00$ & $4.27 \mathrm{E}-01$ & $1.26 \mathrm{E}+00$ & $<34.6$ & $<2.14$ & $5.90 \mathrm{E}-08$ \\
\hline 209-E & $5.04 \mathrm{E}+01$ & $<.61$ & $1.76 \mathrm{E}+01$ & $8.89 E+00$ & $1.96 \mathrm{E}+01$ & $1.18 \mathrm{E}+02$ & $<3.12$ & $5.65 \mathrm{E}-08$ \\
\hline $24 i-S / S X$ & $5.45 E+00$ & $3.17 \mathrm{E}-01$ & $2.17 \mathrm{E}+00$ & & $1.60 \mathrm{E}+00$ & $7.10 \mathrm{E}+01$ & $<2.57$ & $4.45 \mathrm{E}-09$ \\
\hline 241-SY & $5.00 \mathrm{E}+00$ & $2.08 \mathrm{E}+00$ & $2.51 \mathrm{E}+00$ & $3.25 E+00$ & $1.61 \mathrm{E}+00$ & $<36.4$ & $<3.66$ & $4.31 \mathrm{E}-08$ \\
\hline $241-\mathrm{T}$ & $2.76 \mathrm{E}+01$ & $8.56 \mathrm{E}+00$ & $7.77 \mathrm{E}+00$ & $2.58 \mathrm{E}+01$ & $5.60 \mathrm{E}+00$ & $<33.2$ & $7.21 \mathrm{E}+00$ & $<9.28 \mathrm{E}-09$ \\
\hline 241-TX/TY & $2.70 \mathrm{E}+00$ & $<.387$ & $5.47 \mathrm{E}-01$ & & $5.54 \mathrm{E}-01$ & $5.04 \mathrm{E}+01$ & $<1.92$ & $2.75 \mathrm{E}-08$ \\
\hline $241-U$ & $2.39 \mathrm{E}+01$ & $6.18 \mathrm{E}-01$ & $8.80 \mathrm{E}+00$ & $7.19 \mathrm{E}+00$ & $4.71 \mathrm{E}+00$ & $<37.1$ & $<1.83$ & $1.08 \mathrm{E}-07$ \\
\hline & $1.59 \mathrm{E}+01$ & $1.54 \mathrm{E}+00$ & $6.67 \mathrm{E}+00$ & $4.79 \mathrm{E}+00$ & $4.88 \mathrm{E}+00$ & $7.98 \mathrm{E}+01$ & $7.21 \mathrm{E}+00$ & $3.82 \mathrm{E}-08$ \\
\hline Std. Dev. & $1.18 \mathrm{E}+01$ & $2.27 \mathrm{E}+00$ & $3.90 \mathrm{E}+00$ & $6.58 \mathrm{E}+00$ & $4.39 E+00$ & $2.83 \mathrm{E}+01$ & $0.00 \mathrm{E}+00$ & $3.18 \mathrm{E}-08$ \\
\hline $95 \%$ Conf. & $5.78 E+00$ & $1.34 \mathrm{E}+00$ & $1.91 \mathrm{E}+00$ & $3.57 \mathrm{E}+00$ & $2.15 E+00$ & $3.20 \mathrm{E}+01$ & & $1.61 \mathrm{E}-08$ \\
\hline
\end{tabular}

(1) A blank indicates no activity detected

(2) Total Uranium units are $\mathrm{g} / \mathrm{g}$ 


\section{Low Level Waste Sample Set \#5}

pCig (1)

\begin{tabular}{lccccccccc} 
Farm/Area & Total Beta & Total Alpha & Cs-137 & Co-60 & Sr-90 & Tc-99 & Pu-239/240 & Total U (2) \\
\hline \hline & & & & & & & & & \\
$241-\mathrm{AN}$ & $1.72 \mathrm{E}+01$ & $<1.19$ & $4.59 \mathrm{E}+00$ & $4.00 \mathrm{E}+00$ & $2.86 \mathrm{E}+00$ & $<25.4$ & $<1.02$ & $2.62 \mathrm{E}-08$ \\
$241-\mathrm{AP}$ & $7.45 \mathrm{E}+00$ & $1.40 \mathrm{E}+00$ & $3.36 \mathrm{E}+00$ & $3.73 \mathrm{E}+00$ & $1.55 \mathrm{E}+00$ & $<18.1$ & $<.157$ & $5.96 \mathrm{E}-08$ \\
$241-\mathrm{AW}$ & $9.24 \mathrm{E}+00$ & $<2.22$ & $8.74 \mathrm{E}-01$ & & $3.29 \mathrm{E}+00$ & $<61.7$ & $<1.24$ & $7.35 \mathrm{E}-08$ \\
$241-\mathrm{AY}$ & $5.72 \mathrm{E}+00$ & $<1.41$ & $2.70 \mathrm{E}+00$ & $7.66 \mathrm{E}-01$ & $2.58 \mathrm{E}+00$ & $<3.4$ & $<.791$ & $1.95 \mathrm{E}-08$ \\
$244-\mathrm{A}$ & $<1.32$ & $<.739$ & $<.18$ & & $4.20 \mathrm{E}-01$ & $<3.19$ & $<.512$ & $1.06 \mathrm{E}-07$ \\
$242-\mathrm{A}$ & $8.35 \mathrm{E}+00$ & $<.986$ & $1.54 \mathrm{E}+00$ & $1.12 \mathrm{E}+00$ & $2.02 \mathrm{E}+00$ & $<2.85$ & $<.615$ & $8.63 \mathrm{E}-08$ \\
$204-\mathrm{AR}$ & $1.92 \mathrm{E}+01$ & $1.01 \mathrm{E}+00$ & $2.49 \mathrm{E}+00$ & $9.23 \mathrm{E}-01$ & $3.07 \mathrm{E}+00$ & $<3.57$ & $<.332$ & $2.64 \mathrm{E}-07$ \\
$241-\mathrm{B}$ & $5.71 \mathrm{E}+00$ & $<.744$ & $1.14 \mathrm{E}+00$ & $3.60 \mathrm{E}-01$ & $1.34 \mathrm{E}+00$ & $<4.04$ & $<1.31$ & \\
$241-\mathrm{BX} / \mathrm{BY}$ & $2.65 \mathrm{E}+01$ & $<.823$ & $6.90 \mathrm{E}+00$ & $1.80 \mathrm{E}+00$ & $1.06 \mathrm{E}+01$ & $<4.16$ & $<.52$ & \\
$241-\mathrm{C}$ & $2.43 \mathrm{E}+00$ & $<.37$ & $1.88 \mathrm{E}+00$ & $1.54 \mathrm{E}+00$ & $9.02 \mathrm{E}-01$ & $<2.14$ & $<.635$ & $1.20 \mathrm{E}-07$ \\
$209-\mathrm{E}$ & $1.97 \mathrm{E}+01$ & $<.741$ & $5.06 \mathrm{E}+00$ & $8.39 \mathrm{E}+00$ & $6.13 \mathrm{E}+00$ & $<3.41$ & $<.888$ & $2.05 \mathrm{E}-08$ \\
$241-\mathrm{S} / \mathrm{SX}$ & $5.85 \mathrm{E}+00$ & $<.726$ & $1.48 \mathrm{E}+00$ & & $2.26 \mathrm{E}+00$ & $<3.46$ & $4.64 \mathrm{E}-01$ & $4.63 \mathrm{E}-08$ \\
$241-\mathrm{SY}$ & $5.87 \mathrm{E}+00$ & $<.417$ & $1.32 \mathrm{E}+00$ & $4.49 \mathrm{E}-01$ & $2.65 \mathrm{E}+00$ & $1.92 \mathrm{E}+00$ & $<.904$ & $2.74 \mathrm{E}-08$ \\
$241-\mathrm{T}$ & $7.04 \mathrm{E}+00$ & $<.472$ & $2.34 \mathrm{E}+00$ & $3.47 \mathrm{E}-01$ & $3.18 \mathrm{E}+00$ & $<2.31$ & $<.318$ & $3.55 \mathrm{E}-08$ \\
$241-\mathrm{TX} / \mathrm{TY}$ & $5.64 \mathrm{E}+00$ & $<.378$ & $1.48 \mathrm{E}+00$ & $5.14 \mathrm{E}-01$ & $2.63 \mathrm{E}+00$ & $3.18 \mathrm{E}+00$ & $<.597$ & $3.50 \mathrm{E}-08$ \\
$241-\mathrm{U}$ & $5.91 \mathrm{E}+00$ & $<.425$ & $1.32 \mathrm{E}+00$ & & $3.58 \mathrm{E}+00$ & $6.67 \mathrm{E}+00$ & $<.186$ & $2.57 \mathrm{E}-08$ \\
& & & & & & & & \\
& & & & & & & &
\end{tabular}

(1) A blank indicates no activity detected

(2) Total Uranium units are $\mathrm{g} / \mathrm{g}$ 
ATTACHMENT B

WHC-SD-WM-ER-435, Rev. 1 Page B-6 of 29

\section{Low Level Waste Sample Set \#6}

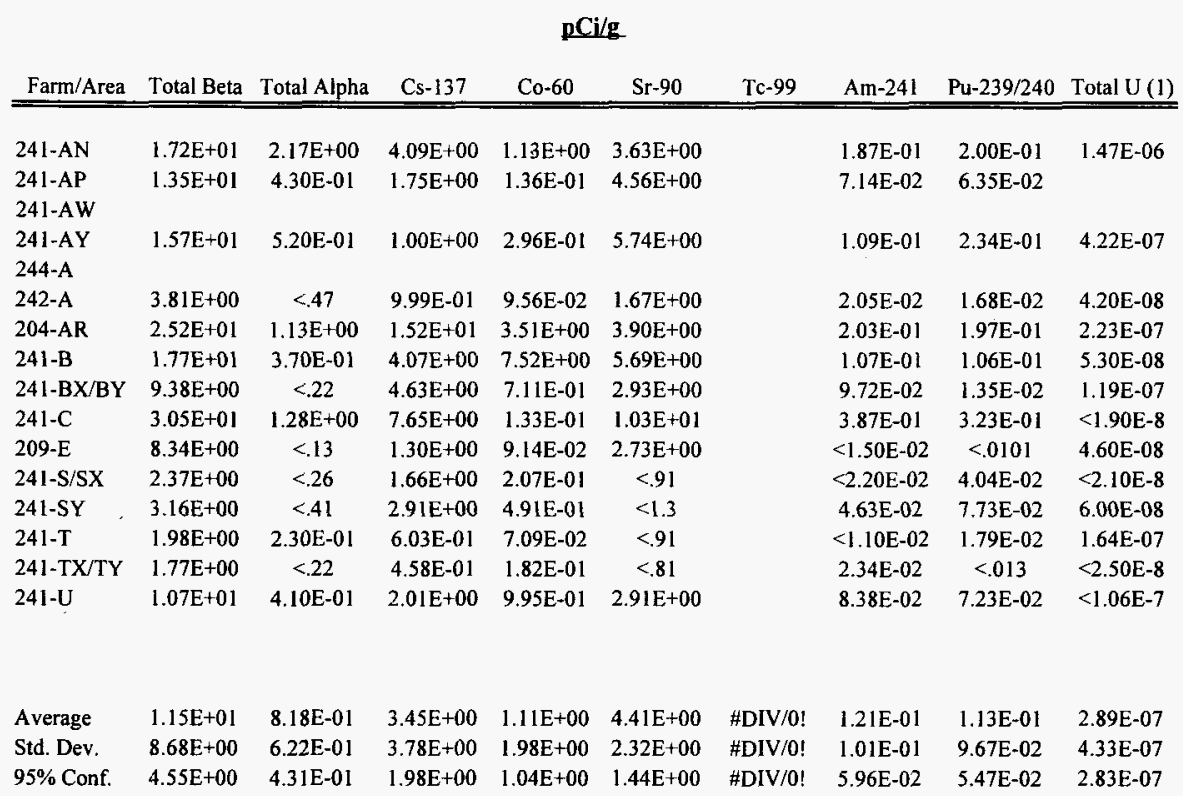

(1) Total Uranium units are $g / g$ 


\section{Low Level Waste Sample Set \#7}

\begin{tabular}{|c|c|c|c|c|c|c|c|c|c|}
\hline Farm/Area & Total Beta & Total Alpha & Cs- 137 & $\mathrm{Co}-60$ & Sr-90 & Tc- 99 & Am-241 & Pu-239/240 & U-238 \\
\hline 241-AN & $1.87 \mathrm{E}+01$ & $5.30 \mathrm{E}-01$ & $5.07 \mathrm{E}+00$ & $6.42 \mathrm{E}+00$ & $4.42 \mathrm{E}+00$ & & $1.16 \mathrm{E}-01$ & $1.48 \mathrm{E}-01$ & 3.94E-02 \\
\hline 241-AP & $1.97 \mathrm{E}+01$ & $3.28 \mathrm{E}+00$ & $7.47 \mathrm{E}+00$ & $2.37 \mathrm{E}-01$ & $4.67 \mathrm{E}+00$ & & $1.33 \mathrm{E}+00$ & $1.00 \mathrm{E}+00$ & $2.78 \mathrm{E}-01$ \\
\hline 241-AW & $2.06 \mathrm{E}+01$ & $1.00 \mathrm{E}+00$ & $3.28 \mathrm{E}+00$ & $6.75 \mathrm{E}-01$ & 7.39E +00 & & $9.30 \mathrm{E}-02$ & $1.20 \mathrm{E}-01$ & $2.44 \mathrm{E}-01$ \\
\hline 241-AY & $6.71 E+00$ & $5.70 \mathrm{E}-01$ & $1.60 E+00$ & $4.02 \mathrm{E}-01$ & $2.01 \mathrm{E} \div 00$ & & $1.08 \mathrm{E}-01$ & $1.26 \mathrm{E}-01$ & $7.71 \mathrm{E}-02$ \\
\hline $244-A$ & $1.52 \mathrm{E}+01$ & $1.61 \mathrm{E}+00$ & $2.64 \mathrm{E}+00$ & $1.01 \mathrm{E}+00$ & $4.14 E+00$ & & $3.91 \mathrm{E}-01$ & $4.97 \mathrm{E}-01$ & $1.95 \mathrm{E}-01$ \\
\hline $242-A$ & $1.50 \mathrm{E}+01$ & $7.20 \mathrm{E}-01$ & $4.39 E+00$ & $1.46 \mathrm{E}+00$ & $3.44 \mathrm{E}+00$ & & $2.23 \mathrm{E}-01$ & $2.70 \mathrm{E}-01$ & $4.15 \mathrm{E}-02$ \\
\hline 204-AR & $1.97 \mathrm{E}+01$ & $2.90 \mathrm{E}+00$ & $2.14 \mathrm{E}+00$ & $7.22 \mathrm{E}+00$ & $3.21 \mathrm{E}+00$ & & $5.60 \mathrm{E}-01$ & $1.98 \mathrm{E}+00$ & $2.08 \mathrm{E}-02$ \\
\hline $241-B$ & $1.05 \mathrm{E}+01$ & $3.90 \mathrm{E}-01$ & $2.30 \mathrm{E}+00$ & $1.88 \mathrm{E}-01$ & $3.19 \mathrm{E}+00$ & & $7.96 \mathrm{E}-02$ & $6.28 \mathrm{E}-02$ & $4.40 \mathrm{E}-02$ \\
\hline 241-BX/BY & $6.74 \mathrm{E}+00$ & $2.20 \mathrm{E}-01$ & $2.08 \mathrm{E}+00$ & $4.57 \mathrm{E}+00$ & $2.38 \mathrm{E}+00$ & & $3.57 \mathrm{E}-02$ & $3.44 \mathrm{E}-02$ & 1.43E-02 \\
\hline $241-\mathrm{C}$ & $6.15 E+00$ & $3.20 \mathrm{E}-01$ & $1.02 \mathrm{E}+00$ & $1.75 \mathrm{E}-01$ & $1.13 \mathrm{E}+00$ & & $4.08 \mathrm{E}-02$ & $2.29 \mathrm{E}-02$ & $6.77 \mathrm{E}-02$ \\
\hline $209-E$ & $3.26 \mathrm{E}+01$ & $2.89 \mathrm{E}+00$ & $8.54 \mathrm{E}+00$ & $2.10 \mathrm{E}+01$ & $5.93 \mathrm{E}+00$ & & $2.71 \mathrm{E}-01$ & $2.67 \mathrm{E}-01$ & 8.13E-01 \\
\hline $241-S / S X$ & $1.07 \mathrm{E}+0 \mathrm{I}$ & $8.40 \mathrm{E}-01$ & $3.45 E+00$ & $4.25 E-01$ & $3.98 \mathrm{E}+00$ & & $2.02 \mathrm{E}-01$ & $3.70 \mathrm{E}-01$ & 9.54E-02 \\
\hline $241-\mathrm{SY}$ & $1.13 E+01$ & $2.90 \mathrm{E}-01$ & $4.81 \mathrm{E}+00$ & $1.03 \mathrm{E}-01$ & $5.70 \mathrm{E}-01$ & & $3.35 \mathrm{E}-02$ & $5.60 \mathrm{E}-02$ & $3.01 \mathrm{E}-02$ \\
\hline $241-T$ & $2.08 \mathrm{E}+00$ & $2.60 \mathrm{E}-01$ & $6.28 \mathrm{E}+00$ & $<.14$ & $5.90 \mathrm{E}-01$ & & $3.79 \mathrm{E}-02$ & $7.75 \mathrm{E}-02$ & $1.20 \mathrm{E}-02$ \\
\hline 241-TX/TY & $1.55 \mathrm{E}+01$ & $<.07$ & $6.88 \mathrm{E}+00$ & $<.12$ & $2.90 \mathrm{E}-01$ & & $1.19 \mathrm{E}-02$ & $8.82 E-03$ & 8. $15 \mathrm{E}-03$ \\
\hline $241-\mathrm{U}$ & $1.40 \mathrm{E}+01$ & $9.90 \mathrm{E}-01$ & $9.23 \mathrm{E}+00$ & $7.75 \mathrm{E}-01$ & $1.91 \mathrm{E}+00$ & & $6.41 \mathrm{E}-02$ & $1.21 \mathrm{E}-01$ & $2.72 \mathrm{E}-01$ \\
\hline Average & $1.41 E+01$ & $1.12 \mathrm{E}+00$ & $4.45 \mathrm{E}+00$ & $3.19 \mathrm{E}+00$ & $3.08 \mathrm{E}+00$ & \#DIV/0! & $2.25 \mathrm{E}-01$ & $3.23 \mathrm{E}-0 \mathrm{I}$ & $1.41 \mathrm{E}-01$ \\
\hline Std. Dev. & $7.16 \mathrm{E}+00$ & $1.02 \mathrm{E}+00$ & $2.49 \mathrm{E}+00$ & $5.46 \mathrm{E}+00$ & $1.94 \mathrm{E}+00$ & \#DIV/0! & $3.20 \mathrm{E}-01$ & $4.92 \mathrm{E}-01$ & $1.97 \mathrm{E}-01$ \\
\hline $95 \%$ Conf. & $3.51 \mathrm{E}+00$ & $5.15 \mathrm{E}-01$ & $1.22 \mathrm{E}+00$ & $2.86 \mathrm{E}+00$ & $9.50 \mathrm{E}-01$ & \#DIV/0! & $1.57 \mathrm{E}-01$ & $2.41 \mathrm{E}-01$ & $9.64 \mathrm{E}-02$ \\
\hline
\end{tabular}




\section{TB \& TA Ratios; Sample Set \#1}

DCi/Sample (1)

\begin{tabular}{lcccccc} 
Farm/Area & Total Beta & Total Alpha & Cs-137 & & \multicolumn{2}{c}{$\begin{array}{c}\text { Ratios } \\
\text { TB/Cs-137 TA/Cs-137 }\end{array}$} \\
\hline 241-A & $6.84 \mathrm{E}+02$ & $7.12 \mathrm{E}+00$ & $6.72 \mathrm{E}+02$ & & 1.02 & 0.011 \\
$241-\mathrm{AN}$ & $1.72 \mathrm{E}+03$ & $<6.53 \mathrm{E}+1$ & $5.66 \mathrm{E}+02$ & & 3.04 & \\
$241-\mathrm{AP}$ & $3.94 \mathrm{E}+03$ & $8.14 \mathrm{E}+02$ & $1.97 \mathrm{E}+03$ & & 2.00 & 0.413 \\
$241-\mathrm{AW}$ & $3.25 \mathrm{E}+03$ & $2.75 \mathrm{E}+01$ & $5.16 \mathrm{E}+03$ & & 0.63 & 0.005 \\
$241-\mathrm{AY}$ & $4.15 \mathrm{E}+03$ & $1.84 \mathrm{E}+01$ & $1.48 \mathrm{E}+03$ & & 2.80 & 0.012 \\
$244-\mathrm{A}$ & $7.56 \mathrm{E}+02$ & $2.50 \mathrm{E}+01$ & $6.21 \mathrm{E}+02$ & & 1.22 & 0.040 \\
$242-\mathrm{A}$ & $2.78 \mathrm{E}+03$ & $2.48 \mathrm{E}+02$ & $1.60 \mathrm{E}+03$ & & 1.74 & 0.155 \\
$204-\mathrm{AR}$ & $2.55 \mathrm{E}+03$ & $8.77 \mathrm{E}+01$ & $1.94 \mathrm{E}+03$ & & 1.31 & 0.045 \\
$241-\mathrm{B}$ & $2.24 \mathrm{E}+03$ & $2.24 \mathrm{E}+01$ & $5.92 \mathrm{E}+02$ & & 3.78 & 0.038 \\
$241-\mathrm{BX} / \mathrm{BY}$ & $2.76 \mathrm{E}+03$ & $3.39 \mathrm{E}+01$ & $2.34 \mathrm{E}+03$ & & 1.18 & 0.014 \\
$241-\mathrm{C}$ & $3.48 \mathrm{E}+04$ & $<1.96 \mathrm{E}+2$ & $2.83 \mathrm{E}+03$ & & 12.30 & \\
$209-\mathrm{E}$ & $6.10 \mathrm{E}+02$ & $1.42 \mathrm{E}+02$ & $1.88 \mathrm{E}+02$ & & 3.24 & 0.755 \\
$241-\mathrm{S} / \mathrm{SX}$ & $2.10 \mathrm{E}+03$ & $<3.22 \mathrm{E}+2$ & $9.59 \mathrm{E}+02$ & & 2.19 & \\
$241-\mathrm{SY}$ & $<1.31 \mathrm{E}+3$ & $<4.93 \mathrm{E}+2$ & $4.79 \mathrm{E}+02$ & & & \\
$241-\mathrm{T}$ & $4.18 \mathrm{E}+03$ & $3.96 \mathrm{E}+02$ & $1.46 \mathrm{E}+03$ & & 2.86 & 0.271 \\
$241-\mathrm{TX} / T \mathrm{TY}$ & $5.25 \mathrm{E}+02$ & $1.24 \mathrm{E}+02$ & $2.24 \mathrm{E}+02$ & & 2.34 & 0.554 \\
$241-\mathrm{U}$ & $4.92 \mathrm{E}+02$ & $<2.85 \mathrm{E}+2$ & $2.86 \mathrm{E}+02$ & & 1.72 &
\end{tabular}

\begin{tabular}{|c|c|c|c|c|c|c|}
\hline Average & $4.22 \mathrm{E}+03$ & $1.62 \mathrm{E}+02$ & $1.37 \mathrm{E}+03$ & \multirow{3}{*}{$+/-$} & \multirow{3}{*}{$\begin{array}{l}3.07 \\
3.23\end{array}$} & \multirow{3}{*}{$\begin{array}{l}0.118 \\
0.110\end{array}$} \\
\hline Std. Dev. & $8.26 E+03$ & $2.36 \mathrm{E}+02$ & $1.26 \mathrm{E}+03$ & & & \\
\hline $95 \%$ Conf. & $4.05 \mathrm{E}+03$ & $1.33 \mathrm{E}+02$ & $5.98 \mathrm{E}+02$ & & & \\
\hline \multicolumn{7}{|c|}{ Without 241-C Data } \\
\hline Average & $2.18 \mathrm{E}+03$ & $1.62 \mathrm{E}+02$ & $1.28 \mathrm{E}+03$ & & 1.70 & 0.126 \\
\hline Std. Dev. & $1.35 \mathrm{E}+03$ & $2.36 \mathrm{E}+02$ & $1.24 \mathrm{E}+03$ & $+/-$ & 0.99 & 0.12 \\
\hline $95 \%$ Conf. & $6.83 E+02$ & $1.33 \mathrm{E}+02$ & $6.28 \mathrm{E}+02$ & & & \\
\hline
\end{tabular}

(1) A blank indicates no activity detected 
TB \& TA Ratios: Sample Set \#2

pCi/g_(1)

\begin{tabular}{|c|c|c|c|c|c|}
\hline Farm/Area & Total Beta & Total Alpha & Cs-137 & TB/Cs- 13 & A/Cs- 137 \\
\hline $241-A$ & $2.92 \mathrm{E}+00$ & $2.19 \mathrm{E}-01$ & $2.21 E+00$ & 1.32 & 0.099 \\
\hline $241-A N$ & $1.28 \mathrm{E}+01$ & $1.70 \mathrm{E}+00$ & $1.80 \mathrm{E}+01$ & 0.71 & 0.094 \\
\hline 241-AP & $1.19 \mathrm{E}+01$ & $6.28 \mathrm{E}-01$ & $1.29 E+01$ & 0.92 & 0.049 \\
\hline $241-A W$ & $2.53 \mathrm{E}+01$ & $1.64 \mathrm{E}+00$ & $1.20 \mathrm{E}+01$ & 2.11 & 0.137 \\
\hline 241-AY & $9.85 E+00$ & $9.75 \mathrm{E}-01$ & $5.57 \mathrm{E}+00$ & 1.77 & 0.175 \\
\hline $244-A$ & $5.80 \mathrm{E}+00$ & $1.27 \mathrm{E}+00$ & $1.58 \mathrm{E}+00$ & 3.67 & 0.804 \\
\hline $242-A$ & $4.70 \mathrm{E}+00$ & $2.50 \mathrm{E}-01$ & $3.20 E+00$ & 1.47 & 0.078 \\
\hline 204-AR & $2.56 \mathrm{E}+00$ & $4.52 \mathrm{E}-0 \mathrm{l}$ & $1.02 \mathrm{E}+00$ & 2.51 & 0.443 \\
\hline $24 t-B$ & $1.80 \mathrm{E}+0 \mathrm{I}$ & $1.94 \mathrm{E}-01$ & $7.43 \mathrm{E}+00$ & 2.42 & 0.026 \\
\hline $241-\mathrm{BX} / \mathrm{BY}$ & $2.90 \mathrm{E}+01$ & $9.98 \mathrm{E}-02$ & $2.14 \mathrm{E}+01$ & 1.36 & 0.005 \\
\hline $241-\mathrm{C}$ & $1.52 \mathrm{E}+01$ & $<9.95 \mathrm{E}-2$ & $7.90 \mathrm{E}+00$ & 1.92 & \\
\hline 209-E & $3.12 E+00$ & $<8.18 \mathrm{E}-2$ & $1.61 \mathrm{E}+00$ & 1.94 & \\
\hline $241-\mathrm{S} / \mathrm{SX}$ & $1.56 \mathrm{E}+01$ & $5.28 \mathrm{E}-01$ & $9.18 \mathrm{E}+00$ & 1.70 & 0.058 \\
\hline 241-SY & $3.04 \mathrm{E}+01$ & $1.08 \mathrm{E}+00$ & $2.81 E+01$ & 1.08 & 0.038 \\
\hline $241-T$ & $7.34 \mathrm{E}+00$ & $<2.12 \mathrm{E}-1$ & $2.44 \mathrm{E}+00$ & 3.01 & \\
\hline 241-TX/TY & $7.10 \mathrm{E}+00$ & $<1.86 \mathrm{E}-1$ & $7.03 E+00$ & 1.01 & \\
\hline $24 \mathrm{I}-\mathrm{U}$ & $1.53 \mathrm{E}+01$ & $4.91 \mathrm{E}-01$ & $1.38 \mathrm{E}+01$ & 1.11 & 0.036 \\
\hline
\end{tabular}

\begin{tabular}{|lllllll|}
\hline Average & $1.28 \mathrm{E}+01$ & $7.33 \mathrm{E}-01$ & $9.14 \mathrm{E}+00$ & & 1.40 & 0.080 \\
Std. Dev. & $\mathbf{8 . 8 7 E}+00$ & $5.48 \mathrm{E}-01$ & $7.75 \mathrm{E}+00$ & $+/-$ & 0.73 & 0.046 \\
$95 \%$ Conf. & $4.21 \mathrm{E}+00$ & $2.98 \mathrm{E}-01$ & $3.69 \mathrm{E}+00$ & & & \\
\hline
\end{tabular}

(1) A blank indicates no activity detected 


\section{TB \& IA Ratios: Sample Set \#3}

\section{pCi/g (1)}

\begin{tabular}{|c|c|c|c|c|c|}
\hline \multirow{2}{*}{$\begin{array}{l}\text { Farm/Area } \\
241-\mathrm{A}\end{array}$} & \multirow{2}{*}{$\frac{\text { Total Beta }}{2.90 \mathrm{E}-01}$} & \multirow{2}{*}{$\begin{array}{c}\text { Total Alpha } \\
<4.83 \mathrm{E}-2\end{array}$} & \multirow{2}{*}{$\underbrace{\mathrm{Cs}-137}_{2.68 \mathrm{E}-01}$} & \multicolumn{2}{|c|}{$\begin{array}{c}\text { Ratios } \\
\text { TB/Cs-137 TA/Cs-137 }\end{array}$} \\
\hline & & & & 1.08 & \\
\hline 241-AN & $4.44 E+02$ & $<7.01 \mathrm{E}-2$ & $5.88 \mathrm{E}-01$ & 755.10 & \\
\hline 241-AP & $7.93 E+00$ & $4.18 \mathrm{E}-01$ & $1.73 E+00$ & 4.58 & 0.242 \\
\hline 241-AW & $9.70 \mathrm{E}+00$ & $<1.01 \mathrm{E}-1$ & $2.07 \mathrm{E}+00$ & 4.69 & \\
\hline 24I-AY & $1.01 \mathrm{E}+00$ & $<4.76 \mathrm{E}-2$ & $3.30 \mathrm{E}-01$ & 3.06 & \\
\hline $244-A$ & $3.67 \mathrm{E}+00$ & $<7.47 \mathrm{E}-2$ & $6.09 \mathrm{E}-01$ & 6.03 & \\
\hline $242-A$ & $1.47 \mathrm{E}+01$ & $1.71 \mathrm{E}-01$ & $4.20 \mathrm{E}+00$ & 3.50 & 0.041 \\
\hline 204-AR & $2.86 \mathrm{E}+01$ & $7.25 \mathrm{E}-01$ & $7.77 \mathrm{E}+00$ & 3.68 & 0.093 \\
\hline $241-B$ & $5.14 \mathrm{E}+01$ & 4.64E-01 & $1.12 \mathrm{E}+01$ & 4.59 & 0.041 \\
\hline 241-BX/BY & $2.04 \mathrm{E}+01$ & $3.09 \mathrm{E}+00$ & $1.37 \mathrm{E}+01$ & 1.49 & 0.226 \\
\hline $241-C$ & $2.88 \mathrm{E}+01$ & $<4.23 \mathrm{E}-2$ & $5.45 \mathrm{E}+01$ & 0.53 & \\
\hline 209-E & $4.88 \mathrm{E}+00$ & $1.23 \mathrm{E}-01$ & $2.84 \mathrm{E}+00$ & 1.72 & 0.043 \\
\hline 241-S/SX & $1.07 \mathrm{E}+00$ & $<7.75 \mathrm{E}-2$ & $9.11 \mathrm{E}-01$ & 1.17 & \\
\hline 241-SY & $4.29 \mathrm{E}+00$ & $<6.82 \mathrm{E}-2$ & $3.25 \mathrm{E}+00$ & 1.32 & \\
\hline $241-T$ & $1.98 \mathrm{E}+00$ & $<7.84 \mathrm{E}-2$ & $1.41 \mathrm{E}+00$ & 1.40 & \\
\hline 241-TX/TY & $9.28 \mathrm{E}+00$ & $2.65 \mathrm{E}-01$ & $6.19 \mathrm{E}+00$ & 1.50 & 0.043 \\
\hline 241-U & $4.40 \mathrm{E}+00$ & $2.03 \mathrm{E}-01$ & $2.66 \mathrm{E}+00$ & 1.65 & 0.076 \\
\hline
\end{tabular}

\begin{tabular}{|lcccccc|}
\hline Average & $3.74 \mathrm{E}+01$ & $6.82 \mathrm{E}-01$ & $6.72 \mathrm{E}+00$ & & 5.57 & 0.102 \\
Std. Dev. & $1.06 \mathrm{E}+02$ & $9.92 \mathrm{E}-01$ & $1.29 \mathrm{E}+01$ & $+/-$ & 9.04 & 0.14 \\
$95 \%$ Conf. & $5.02 \mathrm{E}+01$ & $6.88 \mathrm{E}-01$ & $6.14 \mathrm{E}+00$ & & & \\
\hline
\end{tabular}

\begin{tabular}{|lrlllll|}
\hline Without 241-AN Data & & & & & \\
Average & $1.86 \mathrm{E}+01$ & $7.13 \mathrm{E}-01$ & $7.10 \mathrm{E}+00$ & & 0.100 \\
Std. Dev. & $1.40 \mathrm{E}+01$ & $9.92 \mathrm{E}-01$ & $1.32 \mathrm{E}+01$ \\
$95 \%$ Conf. & $6.85 \mathrm{E}+00$ & $6.88 \mathrm{E}-01$ & $6.49 \mathrm{E}+00$ & +- & 2.58 & 0.133 \\
\hline
\end{tabular}

(1) A blank indicates no activity detected 


\section{TB \& TA Ratios: Sample Set \#4}

\section{pCig (1)}

\section{Ratios}

Farm/Area Total Beta Total Alpha Cs-137

TB/Cs-137 TA/Cs-137

$\begin{array}{lcccccc}241-\mathrm{AN} & 6.14 \mathrm{E}+00 & <.731 & 6.51 \mathrm{E}+00 & 0.94 & \\ 241-\mathrm{AP} & 1.99 \mathrm{E}+01 & 1.18 \mathrm{E}+00 & 6.49 \mathrm{E}+00 & 3.07 & 0.18 \\ 241-\mathrm{AW} & 4.94 \mathrm{E}+00 & <.493 & 4.83 \mathrm{E}+00 & 1.02 & \\ 241-\mathrm{AY} & 1.88 \mathrm{E}+01 & 5.29 \mathrm{E}-01 & 7.36 \mathrm{E}+00 & 2.55 & 0.07 \\ 244-\mathrm{A} & 8.53 \mathrm{E}+00 & 3.74 \mathrm{E}-01 & 3.25 \mathrm{E}+00 & 2.62 & 0.12 \\ 242-\mathrm{A} & 1.93 \mathrm{E}+01 & 7.99 \mathrm{E}-01 & 9.65 \mathrm{E}+00 & 2.00 & 0.08 \\ 204-\mathrm{AR} & 1.79 \mathrm{E}+01 & 3.92 \mathrm{E}-01 & 7.19 \mathrm{E}+00 & 2.49 & 0.05 \\ 241-\mathrm{B} & 2.29 \mathrm{E}+01 & <.381 & 9.45 \mathrm{E}+00 & 2.42 & \\ 241-\mathrm{BX} / \mathrm{BY} & 1.36 \mathrm{E}+01 & 1.21 \mathrm{E}+00 & 8.61 \mathrm{E}+00 & 1.58 & 0.14 \\ 241-\mathrm{C} & 7.46 \mathrm{E}+00 & 8.37 \mathrm{E}-01 & 4.06 \mathrm{E}+00 & 1.84 & 0.21 \\ 209-\mathrm{E} & 5.04 \mathrm{E}+01 & <.61 & 1.76 \mathrm{E}+01 & 2.86 & \\ 241-\mathrm{S} / \mathrm{SX} & 5.45 \mathrm{E}+00 & 3.17 \mathrm{E}-01 & 2.17 \mathrm{E}+00 & 2.51 & 0.15 \\ 241-\mathrm{SY} & 5.00 \mathrm{E}+00 & 2.08 \mathrm{E}+00 & 2.51 \mathrm{E}+00 & 1.99 & 0.83 \\ 241-\mathrm{T} & 2.76 \mathrm{E}+01 & 8.56 \mathrm{E}+00 & 7.77 \mathrm{E}+00 & 3.55 & 1.10 \\ 241-\mathrm{TX} / \mathrm{TY} & 2.70 \mathrm{E}+00 & <.387 & 5.47 \mathrm{E}-01 & 4.94 & \\ 241-\mathrm{U} & 2.39 \mathrm{E}+01 & 6.18 \mathrm{E}-01 & 8.80 \mathrm{E}+00 & 2.72 & 0.07\end{array}$

\begin{tabular}{llll}
\hline Average & $1.59 \mathrm{E}+01$ & $1.54 \mathrm{E}+00$ & $6.67 \mathrm{E}+00$
\end{tabular}

Std. Dev. $\quad 1.18 \mathrm{E}+01 \cdot 2.27 \mathrm{E}+00 \quad 3.90 \mathrm{E}+00$

2.38

0.230

$\begin{array}{llll}95 \% \text { Conf. } & 5.78 \mathrm{E}+00 & 1.34 \mathrm{E}+00 & 1.91 \mathrm{E}+00\end{array}$

$\begin{array}{lll}+/- & 1.10 & 0.212\end{array}$

(1) A blank indicates no activity detected

(2) Total Uranium units are $g / g$ 


\section{TB \& TA Ratios: Sample Set \#5}

\section{pCig (1)}

\begin{tabular}{|c|c|c|c|c|c|}
\hline \multirow{2}{*}{$\begin{array}{l}\text { Farm/Area } \\
\text { 241-AN }\end{array}$} & \multirow{2}{*}{ Total Beta } & \multirow{2}{*}{$\begin{array}{c}\text { Total Alpha } \\
<1.19\end{array}$} & \multirow{2}{*}{$\frac{\mathrm{Cs}-137}{4.59 \mathrm{E}+00}$} & \multicolumn{2}{|c|}{$\frac{\text { Ratios }}{\text { TB/Cs-137 TA/Cs-137 }}$} \\
\hline & & & & 3.75 & \\
\hline 241-AP & $7.45 \mathrm{E}+00$ & $1.40 \mathrm{E}+00$ & $3.36 \mathrm{E}+00$ & 2.22 & 0.417 \\
\hline 241-AW & $9.24 \mathrm{E}+00$ & $<2.22$ & $8.74 \mathrm{E}-01$ & 10.57 & \\
\hline 241-AY & $5.72 \mathrm{E}+00$ & $<1.41$ & $2.70 \mathrm{E}+00$ & 2.12 & \\
\hline $244-A$ & $<1.32$ & $<.739$ & $<.18$ & & \\
\hline $242-A$ & $8.35 E+00$ & $<.986$ & $.1 .54 \mathrm{E}+00$ & 5.42 & \\
\hline 204-AR & $1.92 \mathrm{E}+01$ & $1.01 \mathrm{E}+00$ & $2.49 \mathrm{E}+00$ & 7.71 & 0.406 \\
\hline 241-B & $5.71 \mathrm{E}+00$ & $<.744$ & $1.14 \mathrm{E}+00$ & 5.01 & \\
\hline 241-BX/BY & $2.65 \mathrm{E}+01$ & $<.823$ & $6.90 \mathrm{E}+00$ & 3.84 & \\
\hline $241-C$ & $2.43 E+00$ & $<37$ & $1.88 \mathrm{E}+00$ & 1.29 & \\
\hline 209-E & $1.97 \mathrm{E}+01$ & $<.741$ & $5.06 \mathrm{E}+00$ & 3.89 & \\
\hline 241-S/SX & $5.85 \mathrm{E}+00$ & $<.726$ & $1.48 \mathrm{E}+00$ & 3.95 & \\
\hline $241-S Y$ & $5.87 \mathrm{E}+00$ & $<.417$ & $1.32 \mathrm{E}+00$ & 4.45 & \\
\hline $241-\mathrm{T}$ & $7.04 \mathrm{E}+00$ & $<.472$ & $2.34 \mathrm{E}+00$ & 3.01 & \\
\hline 241-TX/TY & $5.64 E+00$ & $<.378$ & $1.48 \mathrm{E}+00$ & 3.81 & \\
\hline $241-U$ & $5.91 \mathrm{E}+00$ & $<, 425$ & $1.32 \mathrm{E}+00$ & 4.48 & \\
\hline
\end{tabular}

\begin{tabular}{|lllllll|}
\hline Average & $1.0 \mathrm{IE}+01$ & $1.21 \mathrm{E}+00$ & $2.56 \mathrm{E}+00$ & & 3.95 & 0.470 \\
Std. Dev. & $6.76 \mathrm{E}+00$ & $1.95 \mathrm{E}-01$ & $1.67 \mathrm{E}+00$ & $+/-$ & 1.80 & 0.156 \\
$95 \%$ Conf. & $3.31 \mathrm{E}+00$ & $1.15 \mathrm{E}-01$ & $\mathbf{8 . 1 7 \mathrm { E } - 0 1}$ & & & \\
\hline
\end{tabular}

(1) A blank indicates no activity detected

(2) Total Uranium units are $\mathrm{g} / \mathrm{g}$ 


\section{TB \& TA Ratios: Sample Set \#6}

DCi/g.

Ratios

Farm/Area Total Beta Total Alpha $\quad$ Cs-137 $\quad$ TB/Cs-137 TA/Cs-137

\begin{tabular}{lccccc}
\hline \hline & & & & & \\
$241-\mathrm{AN}$ & $1.72 \mathrm{E}+01$ & $2.17 \mathrm{E}+00$ & $4.09 \mathrm{E}+00$ & 4.21 & 0.531 \\
$241-\mathrm{AP}$ & $1.35 \mathrm{E}+01$ & $4.30 \mathrm{E}-0 \mathrm{I}$ & $1.75 \mathrm{E}+00$ & 7.71 & 0.246 \\
$241-\mathrm{AW}$ & & & & & \\
$241-\mathrm{AY}$ & $1.57 \mathrm{E}+01$ & $5.20 \mathrm{E}-01$ & $1.00 \mathrm{E}+00$ & 15.72 & 0.520 \\
$244-\mathrm{A}$ & & & & & \\
$242-\mathrm{A}$ & $3.81 \mathrm{E}+00$ & $<.47$ & $9.99 \mathrm{E}-01$ & 3.81 & \\
$204-\mathrm{AR}$ & $2.52 \mathrm{E}+01$ & $1.13 \mathrm{E}+00$ & $1.52 \mathrm{E}+01$ & 1.66 & 0.074 \\
$241-\mathrm{B}$ & $1.77 \mathrm{E}+01$ & $3.70 \mathrm{E}-01$ & $4.07 \mathrm{E}+00$ & 4.35 & 0.091 \\
$241-\mathrm{BX} / \mathrm{BY}$ & $9.38 \mathrm{E}+00$ & $<.22$ & $4.63 \mathrm{E}+00$ & 2.03 & \\
$241-\mathrm{C}$ & $3.05 \mathrm{E}+01$ & $1.28 \mathrm{E}+00$ & $7.65 \mathrm{E}+00$ & 3.99 & 0.167 \\
$209-\mathrm{E}$ & $8.34 \mathrm{E}+00$ & $<.13$ & $1.30 \mathrm{E}+00$ & 6.42 & \\
$241-\mathrm{S} / \mathrm{SX}$ & $2.37 \mathrm{E}+00$ & $<.26$ & $1.66 \mathrm{E}+00$ & 1.43 & \\
$241-\mathrm{SY}$ & $3.16 \mathrm{E}+00$ & $<.41$ & $2.91 \mathrm{E}+00$ & 1.09 & \\
$241-\mathrm{T}$ & $1.98 \mathrm{E}+00$ & $2.30 \mathrm{E}-01$ & $6.03 \mathrm{E}-01$ & 3.28 & 0.381 \\
$241-\mathrm{TX} / \mathrm{TY}$ & $1.77 \mathrm{E}+00$ & $<.22$ & $4.58 \mathrm{E}-01$ & 3.86 & \\
$241-\mathrm{U}$ & $1.07 \mathrm{E}+01$ & $4.10 \mathrm{E}-01$ & $2.01 \mathrm{E}+00$ & 5.34 & 0.204
\end{tabular}

\begin{tabular}{|lllllll|}
\hline Average & $1.15 \mathrm{E}+01$ & $8.18 \mathrm{E}-01$ & $3.45 \mathrm{E}+00$ & & 3.34 & 0.237 \\
Std. Dev. & $8.68 \mathrm{E}+00$ & $6.22 \mathrm{E}-01$ & $3.78 \mathrm{E}+00$ & $+/-$ & 2.32 & 0.184 \\
$95 \%$ Conf. & $4.55 \mathrm{E}+00$ & $4.31 \mathrm{E}-01$ & $1.98 \mathrm{E}+00$ & & & \\
\hline
\end{tabular}

$95 \%$ Conf. $4.55 \mathrm{E}+00 \quad 4.31 \mathrm{E}-01 \quad 1.98 \mathrm{E}+00$

(1) A blank indicates no activity detected

(2) Total Uranium units are $\mathrm{g} / \mathrm{g}$ 


\title{
TB \& TA Ratios: Sample Set \#7
}

\author{
pCi/g
}

Ratios

Farm/Area Total Beta Total Alpha Cs-137

TB/Cs-137 TA/Cs-137

$\begin{array}{lccccc}241-\mathrm{AN} & 1.87 \mathrm{E}+01 & 5.30 \mathrm{E}-01 & 5.07 \mathrm{E}+00 & 3.68 & 0.105 \\ 241-\mathrm{AP} & 1.97 \mathrm{E}+01 & 3.28 \mathrm{E}+00 & 7.47 \mathrm{E}+00 & 2.64 & 0.439 \\ 241-\mathrm{AW} & 2.06 \mathrm{E}+01 & 1.00 \mathrm{E}+00 & 3.28 \mathrm{E}+00 & 6.27 & 0.305 \\ 241-\mathrm{AY} & 6.71 \mathrm{E}+00 & 5.70 \mathrm{E}-01 & 1.60 \mathrm{E}+00 & 4.19 & 0.356 \\ 244-\mathrm{A} & 1.52 \mathrm{E}+01 & 1.61 \mathrm{E}+00 & 2.64 \mathrm{E}+00 & 5.74 & 0.610 \\ 242-\mathrm{A} & 1.50 \mathrm{E}+01 & 7.20 \mathrm{E}-01 & 4.39 \mathrm{E}+00 & 3.43 & 0.164 \\ 204-\mathrm{AR} & 1.97 \mathrm{E}+01 & 2.90 \mathrm{E}+00 & 2.14 \mathrm{E}+00 & 9.22 & 1.355 \\ 241-\mathrm{B} & 1.05 \mathrm{E}+01 & 3.90 \mathrm{E}-01 & 2.30 \mathrm{E}+00 & 4.54 & 0.170 \\ 241-\mathrm{BX} / \mathrm{BY} & 6.74 \mathrm{E}+00 & 2.20 \mathrm{E}-01 & 2.08 \mathrm{E}+00 & 3.24 & 0.106 \\ 241-\mathrm{C} & 6.15 \mathrm{E}+00 & 3.20 \mathrm{E}-01 & 1.02 \mathrm{E}+00 & 6.03 & 0.314 \\ 209-\mathrm{E} & 3.26 \mathrm{E}+01 & 2.89 \mathrm{E}+00 & 8.54 \mathrm{E}+00 & 3.81 & 0.338 \\ 241-\mathrm{S} / \mathrm{SX} & 1.07 \mathrm{E}+01 & 8.40 \mathrm{E}-01 & 3.45 \mathrm{E}+00 & 3.11 & 0.243 \\ 241-\mathrm{SY} & 1.13 \mathrm{E}+01 & 2.90 \mathrm{E}-01 & 4.81 \mathrm{E}+00 & 2.35 & 0.060 \\ 241-\mathrm{T} & 2.08 \mathrm{E}+00 & 2.60 \mathrm{E}-01 & 6.28 \mathrm{E}+00 & 0.33 & 0.041 \\ 241-\mathrm{TX} / \mathrm{TY} & 1.55 \mathrm{E}+01 & <.07 & 6.88 \mathrm{E}+00 & 2.25 & \\ 241-\mathrm{U} & 1.40 \mathrm{E}+01 & 9.90 \mathrm{E}-01 & 9.23 \mathrm{E}+00 & 1.52 & 0.107\end{array}$

\begin{tabular}{|lllllll|}
\hline Average & $1.41 \mathrm{E}+01$ & $1.12 \mathrm{E}+00$ & $4.45 \mathrm{E}+00$ & & 3.16 & 0.252 \\
Std. Dev. & $7.16 \mathrm{E}+00$ & $1.02 \mathrm{E}+00$ & $2.49 \mathrm{E}+00$ & $+/-$ & 1.17 & 0.135 \\
$95 \%$ Conf. & $3.51 \mathrm{E}+00$ & $5.15 \mathrm{E}-01$ & $1.22 \mathrm{E}+00$ & & & \\
\hline
\end{tabular}

$95 \%$ Conf. $3.51 \mathrm{E}+00 \quad 5.15 \mathrm{E}-01 \quad 1.22 \mathrm{E}+00$

(1) A blank indicates no activity detected

(2) Total Uranium units are $\mathrm{g} / \mathrm{g}$ 


\section{ATTACHMENT B}

WHC-SD-WM-ER-435, Rev. 1

Page B-15 of 29

\section{Total Beta (TB) Ratios}

\section{pCi/g}

\begin{tabular}{|c|c|c|c|c|c|c|c|c|c|c|c|c|}
\hline \multirow[b]{2}{*}{ Farm/Area } & \multicolumn{2}{|c|}{ Sample Set \#2 } & \multicolumn{2}{|c|}{ Sample Set \#3 } & \multicolumn{2}{|c|}{ Sample Set $\# 4$} & \multicolumn{2}{|c|}{ Sample Set $\# 5$} & \multicolumn{2}{|c|}{ Sample Set \#6 } & \multicolumn{2}{|c|}{ Sample Set \#7 } \\
\hline & TB & Cs-137 & TB & $\mathrm{Cs}-137$ & TB & Cs-137 & $\mathrm{TB}$ & $\mathrm{Cs}-137$ & TB & Cs-137 & TB & Cs-137 \\
\hline 241-AN & $28 \mathrm{E}+01$ & $1.80 \mathrm{E}+0 \mathrm{l}$ & $4.22 \mathrm{E}+02$ & $5.88 \mathrm{E}-01$ & $6.14 \mathrm{E}+00$ & $6.51 \mathrm{E}+00$ & $1.72 \mathrm{E}+01$ & $4.59 E+00$ & $1.72 \mathrm{E}+01$ & $4.09 \mathrm{E}+00$ & $1.87 \mathrm{E}+01$ & $5.07 \mathrm{E}+00$ \\
\hline 241-AP & $19 E+01$ & $1.29 \mathrm{E}+01$ & $7.93 \mathrm{E}+00$ & $1.73 \mathrm{E}+00$ & $1.99 \mathrm{E}+01$ & $6.49 \mathrm{E}+00$ & $7.45 \mathrm{E}+00$ & $3.36 \mathrm{E}+00$ & $1.35 \mathrm{E}+01$ & $1.75 \mathrm{E}+00$ & $1.97 \mathrm{E}+01$ & $7.47 \mathrm{E}+00$ \\
\hline $241-A W$ & $53 \mathrm{E}+0 \mathrm{I}$ & $1.20 \mathrm{E}+01$ & $9.70 \mathrm{E}+00$ & $2.07 \mathrm{E}+00$ & $4.94 \mathrm{E}+00$ & $4.83 E+00$ & $9.24 \mathrm{E}+00$ & $8.74 \mathrm{E}-01$ & & & $2.06 \mathrm{E}+01$ & $3.28 \mathrm{E}+00$ \\
\hline $241-$ & $85 \mathrm{E}+00$ & $5.57 \mathrm{E}+00$ & $1.01 \mathrm{E}+00$ & $3.30 \mathrm{E}-01$ & $1.88 \mathrm{E}+01$ & $7.36 \mathrm{E}+00$ & $5.72 \mathrm{E}+00$ & $2.70 \mathrm{E}+00$ & $1.57 \mathrm{E}+0 \mathrm{I}$ & $1.00 \mathrm{E}+00$ & $6.71 E+00$ & $1.60 \mathrm{E}+00$ \\
\hline 244-A & $.80 \mathrm{E}+00$ & $1.58 \mathrm{E}+00$ & $3.67 \mathrm{E}+00$ & 6.05 & $8.53 E+00$ & $3.25 \mathrm{E}+00$ & $<1.32$ & $<.18$ & & & $1.52 \mathrm{E}+01$ & $2.64 \mathrm{E}+00$ \\
\hline $242-\mathrm{A}$ & $4.70 \mathrm{E}+00$ & $3.20 \mathrm{E}+00$ & $1.47 \mathrm{E}+01$ & & & +00 & $8.35 \mathrm{E}+00$ & $1.54 \mathrm{E}+00$ & $3.81 E+00$ & $9.99 \mathrm{E}-01$ & $1.50 E+01$ & $4.39 \mathrm{E}+00$ \\
\hline 204-AR & $2.56 \mathrm{E}+00$ & $1.02 \mathrm{E}+00$ & $2.86 \mathrm{E}+01$ & $7.77 \mathrm{E}+00$ & $1.79 E+01$ & $7.19 \mathrm{E}+00$ & $1.92 \mathrm{E}+01$ & $2.49 \mathrm{E}+00$ & $2.52 \mathrm{E}+01$ & $1.52 \mathrm{E}+01$ & $1.97 \mathrm{E}+$ & $2.14 \mathrm{E}+00$ \\
\hline 241-B & $1.80 \mathrm{E}+01$ & $7.43 \mathrm{E}+00$ & $5.14 \mathrm{E}+01$ & $1.12 \mathrm{E}+01$ & $2.29 \mathrm{E}+01$ & $9.45 \mathrm{E}+00$ & $5.71 E+00$ & $1.14 \mathrm{E}+00$ & $1.77 \mathrm{E}+01$ & $4.07 \mathrm{E}+00$ & $1.05 \mathrm{E}+01$ & $2.30 \mathrm{E}+00$ \\
\hline 241-BX/BY & $2.90 \mathrm{E}+0 \mathrm{I}$ & $2.14 \mathrm{E}+01$ & $2.04 E+01$ & $1.37 \mathrm{E}+01$ & $1.36 \mathrm{E}+01$ & $8.61 \mathrm{E}+00$ & $2.65 \mathrm{E}+01$ & $6.90 \mathrm{E}+00$ & $9.38 \mathrm{E}+00$ & $4.63 E+00$ & $6.74 \mathrm{E}+00$ & $2.08 \mathrm{E}+00$ \\
\hline $241-C$ & $1.52 \mathrm{E}+01$ & $7.90 \mathrm{E}+00$ & $2.88 \mathrm{E}+01$ & $5.45 \mathrm{E}+01$ & $7.46 \mathrm{E}+00$ & $4.06 \mathrm{E}+00$ & $2.43 \mathrm{E}+00$ & $1.88 \mathrm{E}+00$ & $3.05 \mathrm{E}+01$ & $7.65 \mathrm{E}+00$ & $6.15 \mathrm{E}+00$ & $1.02 \mathrm{E}+00$ \\
\hline 209-E & $3.12 E+00$ & $1.61 E+00$ & $4.88 \mathrm{E}+00$ & $2.84 E+00$ & $5.04 \mathrm{E}+01$ & $1.76 \mathrm{E}+01$ & $1.97 \mathrm{E}+01$ & $5.06 \mathrm{E}+00$ & $8.34 \mathrm{E}+00$ & $1.30 \mathrm{E}+00$ & $3.26 \mathrm{E}+01$ & $8.54 E+00$ \\
\hline 241-S/SX & $1.56 \mathrm{E}+01$ & $9.18 \mathrm{E}+00$ & $1.07 \mathrm{E}+00$ & $9.11 \mathrm{E}-01$ & $5.45 E+00$ & $2.17 \mathrm{E}+00$ & $5.85 \mathrm{E}+00$ & $1.48 \mathrm{E}+00$ & $2.37 \mathrm{E}+00$ & $1.66 \mathrm{E}+00$ & $1.07 \mathrm{E}+01$ & $3.45 \mathrm{E}+00$ \\
\hline 241-SY & $3.04 \mathrm{E}+01$ & $2.81 E+01$ & $4.29 E+00$ & $3.25 \mathrm{E}+00$ & $5.00 \mathrm{E}+00$ & $2.51 \mathrm{E}+00$ & $5.87 \mathrm{E}+00$ & $1.32 \mathbf{E}+00$ & $3.16 \mathrm{E}+00$ & $2.91 E+00$ & $1.13 \mathrm{E}+01$ & $4.81 \mathrm{E}+00$ \\
\hline $241-\mathrm{T}$ & $7.34 \mathrm{E}+00$ & $2.44 \mathrm{E}+00$ & $1.98 \mathrm{E}+00$ & $1.41 \mathrm{E}+00$ & $2.76 \mathrm{E}+01$ & 7.77E+00 & $7.04 \mathrm{E}+00$ & $2.34 \mathrm{E}+00$ & $1.98 \mathrm{E}+00$ & 6.03E-01 & $2.08 \mathrm{E}+00$ & $6.28 \mathrm{E}+00$ \\
\hline 241-TX/TY & $7.10 \mathrm{E}+00$ & $7.03 E+00$ & $9.28 \mathrm{E}+00$ & $6.19 \mathrm{E}+00$ & $2.70 \mathrm{E}+00$ & $5.47 \mathrm{E}-01$ & $5.64 \mathrm{E}+00$ & $1.48 \mathrm{E}+00$ & $1.77 \mathrm{E}+00$ & $4.58 \mathrm{E}-01$ & $1.55 \mathrm{E}+01$ & $6.88 \mathrm{E}+00$ \\
\hline $241-U$ & $1.53 E+01$ & $1.38 E+01$ & $4.40 E+00$ & $2.66 \mathrm{E}+00$ & $2.39 \mathrm{E}+01$ & $8.80 E+00$ & $5.91 E+00$ & $1.32 \mathrm{E}+00$ & $1.07 E+01$ & $2.01 \mathrm{E}+00$ & $1.40 \mathrm{E}+01$ & $9.23 E+00$ \\
\hline
\end{tabular}

Ratio Using All Individual Activities Without 241-AN Set \#3

$\begin{array}{lcccc} & \text { TB } & \text { Cs-137 } & \\ \text { Average } & 13.03 & 5.72 & & 2.279 \\ \text { Std. Dev. } & 9.91 & 7.07 & +/- & 0.676 \\ 95 \% \text { Conf. } & 2.03 & 1.45 & & \end{array}$




\section{Total Alpha (TA) Ratios}

pCi/g

\begin{tabular}{|c|c|c|c|c|c|c|c|c|c|c|c|c|}
\hline \multirow[b]{2}{*}{ Farm/Area } & \multicolumn{2}{|c|}{ Sample Set \#2 } & \multicolumn{2}{|c|}{ Sample Set \#3 } & \multicolumn{2}{|c|}{ Sample Set \#4 } & \multicolumn{2}{|c|}{ Sample Set \#5 } & \multicolumn{2}{|c|}{ Sample Set $\# 6$} & \multicolumn{2}{|c|}{ Sample Sat \#7 } \\
\hline & TA & Cs-137 & TA & Cs-137 & TA & Cs-137 & TA & Cs-137 & $\mathrm{TA}$ & Cs-137 & TA & Cs-137 \\
\hline 241-AN & $70 \mathrm{E}+00$ & $1.80 \mathrm{E}+01$ & $<.070$ & $5.88 \mathrm{E}-01$ & $<.731$ & $6.51 \mathrm{E}+00$ & $<1.19$ & $4.59 E+00$ & $2.17 \mathrm{E}+00$ & $4.09 \mathrm{E}+00$ & $5.30 \mathrm{E}-01$ & $5.07 \mathrm{E}+00$ \\
\hline 241-AP & $28 \mathrm{E}-01$ & $1.29 \mathrm{E}+01$ & $4.18 \mathrm{E}-01$ & $1.73 E+00$ & $1.18 \mathrm{E}+00$ & $6.49 \mathrm{E}+00$ & $1.40 \mathrm{E}+00$ & $3.36 \mathrm{E}+00$ & 4.30E-01 & $1.75 \mathrm{E}+00$ & $3.28 \mathrm{E}+00$ & $7.47 \mathrm{E}+00$ \\
\hline $241-A$ & $1.64 \mathrm{E}+00$ & $1.20 \mathrm{E}+01$ & $<.101$ & $2.07 \mathrm{E}+00$ & $<.493$ & $4.83 E+00$ & $<2.22$ & $8.74 \mathrm{E}-01$ & & & $1.00 \mathrm{E}+00$ & $3.28 \mathrm{E}+00$ \\
\hline 241-AY & $9.75 \mathrm{E}-01$ & $5.57 \mathrm{E}+00$ & $<048$ & $3.30 \mathrm{E}-01$ & $5.29 \mathrm{E}-01$ & $7.36 \mathrm{E}+00$ & $<1.41$ & $2.70 \mathrm{E}+00$ & $5.20 \mathrm{E}-01$ & $1.00 \mathrm{E}+00$ & $5.70 \mathrm{E}-01$ & $1.60 \mathrm{E}+00$ \\
\hline $244-A$ & $1.27 \mathrm{E}+00$ & $1.58 \mathrm{E}+00$ & $<.075$ & $6.09 \mathrm{E}-01$ & 3.74E-01 & $3.25 E+00$ & $<.739$ & $<18$ & & & $1.61 \mathrm{E}+00$ & $2.64 \mathrm{E}+00$ \\
\hline $242-A$ & $.50 \mathrm{E}-01$ & $3.20 \mathrm{E}+00$ & $1.71 \mathrm{E}-01$ & $4.20 \mathrm{E}+00$ & $7.99 \mathrm{E}-01$ & $9.65 E+00$ & $<.986$ & $1.54 \mathrm{E}+00$ & $<.47$ & $9.99 \mathrm{E}-01$ & $7.20 \mathrm{E}-01$ & 4.39E+00 \\
\hline $204-$ & $52 \mathrm{E}-01$ & $1.02 E+00$ & $7.25 \mathrm{E}-01$ & $7.77 \mathrm{E}+00$ & $3.92 \mathrm{E}-01$ & $7.19 \mathrm{E}+00$ & $1.01 \mathrm{E}+00$ & $2.49 \mathrm{E}+00$ & $1.13 E+00$ & $1.52 \mathrm{E}+01$ & $2.90 \mathrm{E}+00$ & $2.14 \mathrm{E}+00$ \\
\hline 241-B & $1.94 \mathrm{E}-0 \mathrm{l}$ & $7.43 \mathrm{E}+00$ & $4.64 \mathrm{E}-0 \mathrm{l}$ & $1.12 \mathrm{E}+01$ & $<381$ & $9.45 \mathrm{E}+00$ & $<.744$ & 1. $14 \mathrm{E}+00$ & $3.70 \mathrm{E}-01$ & $4.07 \mathrm{E}+00$ & $3.90 \mathrm{E}-01$ & $2.30 \mathrm{E}+00$ \\
\hline $241 \cdot B X / B Y$ & $9.98 \mathrm{E}-02$ & $2.14 \mathrm{E}+01$ & $3.09 E+00$ & $1.37 \mathrm{E}+01$ & $1.21 \mathrm{E}+00$ & $8.61 \mathrm{E}+00$ & $<.823$ & $6.90 \mathrm{E}+00$ & $<.22$ & $4.63 E+00$ & $2.20 \mathrm{E}-01$ & $2.08 \mathrm{E}+00$ \\
\hline $241-C$ & $<.099$ & $7.90 \mathrm{E}+00$ & $<.042$ & $5.45 E+01$ & 8.37E-01 & $4.06 E+00$ & $<.37$ & $1.88 E+00$ & $1.28 \mathrm{E}+00$ & $7.65 \mathrm{E}+00$ & $3.20 \mathrm{E}-01$ & $1.02 E+00$ \\
\hline 209-E & $<.082$ & $1.61 \mathrm{E}+00$ & $1.23 \mathrm{E}-01$ & $2.84 \mathrm{E}+00$ & $<.61$ & $1.76 \mathrm{E}+01$ & $<.741$ & $5.06 \mathrm{E}+00$ & $<.13$ & $1.30 \mathrm{E}+00$ & $2.89 \mathrm{E}+00$ & $8.54 \mathrm{E}+00$ \\
\hline & $5.28 \mathrm{E}-01$ & $9.18 \mathrm{E}+00$ & $<.078$ & $9.11 \mathrm{E}-01$ & $3.17 \mathrm{E}-01$ & $2.17 \mathrm{E}+00$ & $<.726$ & $1.48 \mathrm{E}+00$ & $<.26$ & $1.66 \mathrm{E}+00$ & $8.40 \mathrm{E}-01$ & $3.45 \mathrm{E}+00$ \\
\hline 241-SY & $1.08 \mathrm{E}+00$ & $2.81 \mathrm{E}+01$ & $<.068$ & $3.25 \mathrm{E}+00$ & $2.08 \mathrm{E}+00$ & $2.51 \mathrm{E}+00$ & $<.417$ & $1.32 E+00$ & $<.41$ & $2.91 \mathrm{E}+00$ & $2.90 \mathrm{E}-01$ & $4.81 \mathrm{E}+00$ \\
\hline & $<.212$ & $2.44 E+00$ & $<.078$ & $1.41 E+00$ & $8.56 \mathrm{E}+00$ & $7.77 \mathrm{E}+00$ & $<.472$ & $2.34 E+00$ & $2.30 \mathrm{E}-01$ & $6.03 E-01$ & $2.60 \mathrm{E}-0 \mathrm{I}$ & $6.28 \mathrm{E}+00$ \\
\hline & $<.186$ & $7.03 \mathrm{E}+00$ & $2.65 \mathrm{E}-01$ & $6.19 \mathrm{E}+00$ & $<.387$ & $5.47 \mathrm{E}-01$ & $<.378$ & $1.48 \mathrm{E}+00$ & $<.22$ & $4.58 \mathrm{E}-01$ & $<.07$ & $6.88 \mathrm{E}+00$ \\
\hline $241-\mathrm{U}$ & $4.91 E-01$ & $1.38 \mathrm{E}+01$ & $2.03 \mathrm{E}-01$ & $2.66 \mathrm{E}+00$ & $6.18 E-01$ & $8.80 E+00$ & $<.425$ & $1.32 \mathrm{E}+00$ & $4.10 \mathrm{E}-01$ & $2.01 E+00$ & $9.90 \mathrm{E}-01$ & $9.23 \mathrm{E}+00$ \\
\hline
\end{tabular}

\begin{tabular}{|c|c|c|c|c|}
\hline \multicolumn{5}{|c|}{ Ratio Using All Individual Activities } \\
\hline & TA & Cs- 137 & & Ratio \\
\hline Average & 1.03 & 5.72 & & 0.179 \\
\hline Std. Dev. & 1.27 & 7.07 & $+/-$ & 0.074 \\
\hline $95 \%$ Conf. & 0.34 & 1.44 & & \\
\hline
\end{tabular}




\section{Sr-90Ratios}

$\mathrm{pCi} / \mathrm{g}$

\begin{tabular}{l|cc|cc|cc|cc|cc} 
& \multicolumn{2}{|c|}{ Sample Set \#3 } & \multicolumn{2}{c|}{ Sample Set \#4 } & \multicolumn{2}{c}{ Sample Set \#5 } & \multicolumn{2}{c}{ Sample Set \#6 } & Sample Set \#7 \\
Farm/Area & Sr-90 & Cs-137 & Sr-90 & Cs-137 & Sr-90 & Cs-137 & Sr-90 & Cs-137 & Sr-90 \\
Cs-137
\end{tabular}

\begin{tabular}{|c|c|c|c|c|}
\hline \multicolumn{5}{|c|}{ Ratio Using All Individual Activities } \\
\hline & Sr-90 & Cs-137 & & Ratio \\
\hline Average & 4.04 & 5.04 & \multirow{3}{*}{$+1-$} & $0 . \overline{801}$ \\
\hline Std. Dev. & 3.41 & 7.91 & & 0.369 \\
\hline $95 \%$ Conf. & 0.97 & 1.98 & & \\
\hline
\end{tabular}




\section{Tc-99 Ratios}

pCi/g.

\begin{tabular}{|c|c|c|c|c|c|c|}
\hline \multirow[b]{2}{*}{ Farm/Area } & \multicolumn{2}{|c|}{ Sample Set \#4 } & \multicolumn{2}{|c|}{ Sample Set \#5 } & Sample Set \#6 & Sample Set \#7 \\
\hline & Tc-99 & Cs-137 & Tc-99 & Cs-137 & Tc-99 Cs- 137 & Tc-99 Cs-137 \\
\hline 241-AN & $<35.8$ & $6.51 E+00$ & $<25.4$ & $4.59 \mathrm{E}+00$ & $4.09 E+00$ & $5.07 \mathrm{E}+00$ \\
\hline 241-AP & $<32.9$ & $6.49 \mathrm{E}+00$ & $<18.1$ & $3.36 \mathrm{E}+00$ & $1.75 \mathrm{E}+00$ & $7.47 \mathrm{E}+00$ \\
\hline 241-AW & $<41.8$ & $4.83 \mathrm{E}+00$ & $<61.7$ & 8.74E-01 & & $3.28 \mathrm{E}+00$ \\
\hline 241-AY & $<26.9$ & $7.36 \mathrm{E}+00$ & $<3.4$ & $2.70 \mathrm{E}+00$ & $1.00 \mathrm{E}+00$ & $1.60 \mathrm{E}+00$ \\
\hline $244-\mathrm{A}$ & $<32.8$ & $3.25 \mathrm{E}+00$ & $<3.19$ & $<.18$ & & $2.64 \mathrm{E}+00$ \\
\hline $242-A$ & $<29.1$ & $9.65 E+00$ & $<2.85$ & $1.54 \mathrm{E}+00$ & 9.99E-01 & $4.39 \mathrm{E}+00$ \\
\hline 204-AR & $<32.6$ & $7.19 \mathrm{E}+00$ & $<3.57$ & $2.49 \mathrm{E}+00$ & $1.52 \mathrm{E}+01$ & $2.14 \mathrm{E}+00$ \\
\hline $241-B$ & $<33.3$ & $9.45 E+00$ & $<4.04$ & $1.14 \mathrm{E}+00$ & $4.07 \mathrm{E}+00$ & $2.30 \mathrm{E}+00$ \\
\hline 241-BX/BY & $<38$ & $8.61 E+00$ & $<4.16$ & $6.90 \mathrm{E}+00$ & $4.63 \mathrm{E}+00$ & $2.08 \mathrm{E}+00$ \\
\hline $241-\mathrm{C}$ & $<34.6$ & $4.06 \mathrm{E}+00$ & $<2.14$ & $1.88 \mathrm{E}+00$ & $7.65 \mathrm{E}+00$ & $1.02 \mathrm{E}+00$ \\
\hline 209-E & $1.18 E+02$ & $1.76 \mathrm{E}+01$ & $<3.41$ & $5.06 \mathrm{E}+00$ & $1.30 \mathrm{E}+00$ & $8.54 \mathrm{E}+00$ \\
\hline $241-S / S X$ & $7.10 \mathrm{E}+01$ & $2.17 \mathrm{E}+00$ & $<3.46$ & $1.48 \mathrm{E}+00$ & $1.66 \mathrm{E}+00$ & $3.45 \mathrm{E}+00$ \\
\hline 241-SY & $<36.4$ & $2.51 \mathrm{E}+00$ & $1.92 \mathrm{E}+00$ & $1.32 \mathrm{E}+00$ & $2.91 \mathrm{E}+00$ & $4.81 E+00$ \\
\hline $241-T$ & $<33.2$ & $7.77 \mathrm{E}+00$ & $<2.31$ & $2.34 \mathrm{E}+00$ & $6.03 \mathrm{E}-01$ & $6.28 \mathrm{E}+00$ \\
\hline 241-TX/TY & $5.04 \mathrm{E}+01$ & $5.47 \mathrm{E}-01$ & $3.18 \mathrm{E}+00$ & $1.48 \mathrm{E}+00$ & $4.58 \mathrm{E}-01$ & $6.88 \mathrm{E}+00$ \\
\hline $241-U$ & $<37.1$ & $8.80 \mathrm{E}+00$ & $6.67 \mathrm{E}+00$ & $1.32 \mathrm{E}+00$ & $2.01 \mathrm{E}+00$ & $9.23 \mathrm{E}+00$ \\
\hline
\end{tabular}

\begin{tabular}{|c|c|c|c|c|}
\hline \multicolumn{5}{|c|}{ Ratio Using All Individual Activities } \\
\hline & Tc-99 & Cs- 137 & & Ratio \\
\hline Average & $\overline{\overline{41.86}}$ & 4.34 & & $\overline{9.644}$ \\
\hline Std. Dev. & 42.91 & 3.46 & $+\%$ & 3.398 \\
\hline $95 \%$ Conf & 12.14 & 0.87 & & \\
\hline
\end{tabular}




\section{U-234 Ratio Cales for LLW}

\begin{tabular}{|c|c|c|c|c|c|c|c|c|c|c|c|c|}
\hline \multirow[b]{2}{*}{ Farm/Area } & \multicolumn{3}{|c|}{ Sample Set \#4 } & \multicolumn{3}{|c|}{ Sample Set \#5 } & \multicolumn{3}{|c|}{ Sample Set \#6 } & \multicolumn{3}{|c|}{ Sample Set \#7 } \\
\hline & $\mathrm{J}-234$ & Cs-137 & Ratio & U-234 & Cs-137 & Ratio & $\mathrm{U}-234$ & Cs- 137 & Ratio & $\mathrm{U}-234$ & Cs-137 & Ratio \\
\hline $241-$ & $37 \mathrm{E}-07$ & $6.51 \mathrm{E}+00$ & $2.10 \mathrm{E}-08$ & $8.02 E-07$ & $4.59 \mathrm{E}+00$ & $1.75 \mathrm{E}-07$ & $4.50 \mathrm{E}-05$ & $4.09 \mathrm{E}+00$ & $1.10 \mathrm{E}-05$ & 6.82E-05 & $5.07 \mathrm{E}+00$ & 1.3 \\
\hline & 47E-07 & $6.49 \mathrm{E}+00$ & -08 & $1.82 \mathrm{E}-06$ & 3.36 & -07 & $9.83 \mathrm{E}-05$ & $1.75 \mathrm{E}+00$ & -05 & $4.81 \mathrm{E}-04$ & +00 & -05 \\
\hline $241-$ & $90 \mathrm{E}-06$ & $4.83 E+00$ & $6.00 \mathrm{E}-07$ & $2.25 \mathrm{E}-06$ & $8.74 \mathrm{E}-01$ & $2.57 \mathrm{E}-06$ & & & & $4.22 E-04$ & $3.28 \mathrm{E}+00$ & -04 \\
\hline $241-A$ & $18 \mathrm{E}-07$ & $7.36 \mathrm{E}+00$ & 4.33 & $5.97 \mathrm{E}-07$ & $2.70 \mathrm{E}+00$ & $2.21 \mathrm{E}-07$ & $1.29 \mathrm{E}-05$ & $1.00 \mathrm{E}+00$ & 1.29E-05 & $1.33 \mathrm{E}-04$ & $1.60 \mathrm{E}+00$ & \\
\hline 244-A & $.07 \mathrm{E}-06$ & $3.25 \mathrm{E}+00$ & $6.38 \mathrm{E}-07$ & $3.24 \mathrm{E}-06$ & $<.18$ & & & & & $3.37 \mathrm{E}-04$ & $2.64 \mathrm{E}+00$ & -04 \\
\hline $242-$ & $7.19 \mathrm{E}-07$ & $9.65 \mathrm{E}+00$ & $7.45 \mathrm{E}-08$ & $2.64 \mathrm{E}-06$ & $1.54 \mathrm{E}+00$ & $1.72 \mathrm{E}-06$ & $1.29 \mathrm{E}-06$ & $9.99 \mathrm{E}-01$ & $1.29 \mathrm{E}-06$ & $7.18 \mathrm{E}-05$ & $4.39 \mathrm{E}+00$ & $1.64 \mathrm{E}-05$ \\
\hline & $1.11 \mathrm{E}-06$ & $7.19 \mathrm{E}+00$ & $1.55 \mathrm{E}-07$ & $8.08 E-06$ & $2.49 \mathrm{E}+00$ & $3.25 \mathrm{E}-06$ & $6.83 \mathrm{E}-06$ & $1.52 \mathrm{E}+0 \mathrm{I}$ & $E-07$ & $3.60 \mathrm{E}-05$ & +00 & $1.68 \mathrm{E}-05$ \\
\hline & -07 & $9.45 \mathrm{E}+00$ & $6.06 \mathrm{E}-08$ & & $1.14 \mathrm{E}+00$ & & $1.62 \mathrm{E}-06$ & $4.07 \mathrm{E}+00$ & $\mathrm{E}-07$ & $7.61 \mathrm{E}-05$ & $+\infty$ & $3.31 \mathrm{E}-05$ \\
\hline & & & & & 6.901 & & $3.64 \mathrm{E}-06$ & $4.63 \mathrm{E}+00$ & $7.87 \mathrm{E}-07$ & $2.47 \mathrm{E}-05$ & +00 & $1.19 \mathrm{E}-05$ \\
\hline & $1.81 \mathrm{E}-06$ & $4.06 \mathrm{E}+00$ & $4.45 \mathrm{E}-07$ & $3.67 \mathrm{E}-06$ & $1.88 \mathrm{E}+00$ & $1.95 \mathrm{E}-06$ & & $7.65 \mathrm{E}+00$ & & $1.17 \mathrm{E}-04$ & +00 & $5 E-04$ \\
\hline 209-E & $1.73 \mathrm{E}-06$ & $1.76 E+01$ & $9.83 \mathrm{E}-08$ & $6.28 \mathrm{E}-07$ & $5.06 \mathrm{E}+00$ & $1.24 \mathrm{E}-07$ & $1.41 \mathrm{E}-06$ & $1.30 \mathrm{E}+00$ & $1.08 \mathrm{E}-06$ & $1.41 \mathrm{E}-03$ & +00 & $1.65 \mathrm{E}-04$ \\
\hline & $1.36 \mathrm{E}-07$ & $2.17 \mathrm{E}+00$ & $6.28 \mathrm{E}-08$ & $1.42 \mathrm{E}-06$ & $1.48 \mathrm{E}+00$ & $9.58 \mathrm{E}-07$ & & $1.66 \mathrm{E}+00$ & & $1.65 \mathrm{E}-04$ & $3.45 E+00$ & $4.78 \mathrm{E}-05$ \\
\hline & 1.32E-06 & $2.51 \mathrm{E}+00$ & $5.26 \mathrm{E}-07$ & 8.39E-07 & $1.32 \mathrm{E}+00$ & $6.35 \mathrm{E}-07$ & $1.84 \mathrm{E}-06$ & $2.91 \mathrm{E}+00$ & $6.31 \mathrm{E}-07$ & $5.21 \mathrm{E}-05$ & $4.81 E+00$ & $1.08 \mathrm{E}-05$ \\
\hline & & $7.77 \mathrm{E}+00$ & & $1.09 \mathrm{E}-06$ & $2.34 \mathrm{E}+00$ & $4.64 \mathrm{E}-07$ & $5.02 \mathrm{E}-06$ & $6.03 \mathrm{E}-01$ & 8.33E-06 & $2.08 \mathrm{E}-05$ & $6.28 E+00$ & $3.31 \mathrm{E}-06$ \\
\hline & 8.42E-07 & $5.47 \mathrm{E}-01$ & $1.54 \mathrm{E}-06$ & $1.07 \mathrm{E}-06$ & $1.48 E+00$ & $7.24 \mathrm{E}-07$ & & $4.58 \mathrm{E}-01$ & & $1.41 \mathrm{E}-05$ & $6.88 \mathrm{E}+00$ & $2.05 \mathrm{E}-06$ \\
\hline $241-\mathrm{U}$ & $3.31 \mathrm{E}-06$ & $8.80 E+00$ & $3.76 \mathrm{E}-07$ & $7.87 \mathrm{E}-07$ & $1.32 \mathrm{E}+00$ & $5.96 \mathrm{E}-07$ & & $2.01 \mathrm{E}+00$ & & $4.71 E-04$ & $9.23 E+00$ & $5.10 \mathrm{E}-05$ \\
\hline
\end{tabular}

(1) A blank indicates no activity detected

\begin{tabular}{|c|c|c|c|}
\hline \multicolumn{4}{|c|}{ Ratio Using All Individual Activities } \\
\hline & U-234 & Cs-137 & Ratio \\
\hline Average & $7.49 \mathrm{E}-05$ & $4.34 \mathrm{E}+00$ & $1.73 \mathrm{E}-05$ \\
\hline Std. Dev. & $2.13 \mathrm{E}-04$ & $3.46 \mathrm{E}+00$ & $+1.34 \mathrm{E}-05$ \\
\hline $95 \%$ Conf & $5.64 \mathrm{E}-05$ & $8.68 \mathrm{E}-01$ & \\
\hline
\end{tabular}




\section{U-235 Ratio Cales for LLW}

\begin{tabular}{|c|c|c|c|c|c|c|c|c|c|c|c|c|}
\hline \multirow[b]{2}{*}{ Farm/Area } & \multicolumn{3}{|c|}{ Sample Set \#4 } & \multicolumn{3}{|c|}{ Sample Set $\# 5$} & \multicolumn{3}{|c|}{ Sample Set \#6 } & \multicolumn{3}{|c|}{ Sample Set $\# 7$} \\
\hline & $\mathrm{U}-235$ & Cs-137 & Ratio & $\mathrm{U}-235$ & Cs-137 & Ratio & $\mathrm{U}-235$ & Cs- 137 & Ratio & U-235 & Cs- 137 & Ratio \\
\hline 241-AN & $16 \mathrm{E}-05$ & $6.51 \mathrm{E}+00$ & $.25 \mathrm{E}-05$ & $78 \mathrm{E}-04$ & $.59 \mathrm{E}+00$ & $1.04 \mathrm{E}-04$ & 2.68E-02 & $4.09 E+00$ & $6.56 \mathrm{E}-03$ & $2.16 \mathrm{E}-03$ & $5.07 E+00$ & $4.26 \mathrm{E}-04$ \\
\hline 241-AP & 47E-04 & $6.49 \mathrm{E}+00$ & 2.27E-05 & $1.09 \mathrm{E}-03$ & $3.36 \mathrm{E}+00$ & $3.24 \mathrm{E}-04$ & $3.11 \mathrm{E}-03$ & $1.75 \mathrm{E}+00$ & $1.78 \mathrm{E}-03$ & $1.52 \mathrm{E}-02$ & $7.47 \mathrm{E}+00$ & $2.04 \mathrm{E}-03$ \\
\hline 241-AW & $73 \mathrm{E}-03$ & $4.83 E+00$ & $3.58 \mathrm{E}-04$ & $1.34 \mathrm{E}-03$ & $8.74 \mathrm{E}-01$ & $1.54 \mathrm{E}-03$ & & & & $1.34 \mathrm{E}-02$ & $3.28 \mathrm{E}+00$ & 4.07E-03 \\
\hline 241-AY & $.90 \mathrm{E}-04$ & $7.36 \mathrm{E}+00$ & $2.58 \mathrm{E}-05$ & $3.56 \mathrm{E}-04$ & $2.70 \mathrm{E}+00$ & 1.32E-04 & $7.71 \mathrm{E}-03$ & $1.00 \mathrm{E}+00$ & $7.71 \mathrm{E}-03$ & $4.22 \mathrm{E}-03$ & $1.60 \mathrm{E}+00$ & $2.64 \mathrm{E}-03$ \\
\hline $244-A$ & $1.24 \mathrm{E}-03$ & $3.25 \mathrm{E}+00$ & $3.80 \mathrm{E}-04$ & $1.94 \mathrm{E}-03$ & $<.18$ & & & & & $1.07 \mathrm{E}-02$ & $2.64 \mathrm{E}+00$ & $4.05 \mathrm{E}-03$ \\
\hline 242 & 4.29E-04 & $9.65 \mathrm{E}+00$ & $4.45 \mathrm{E}-05$ & $1.58 \mathrm{E}-03$ & $1.54 \mathrm{E}+00$ & $1.02 \mathrm{E}-03$ & $7.67 \mathrm{E}-04$ & $9.99 \mathrm{E}-01$ & $7.68 \mathrm{E}-04$ & $2.27 \mathrm{E}-03$ & $4.39 \mathrm{E}+00$ & $5.18 \mathrm{E}-04$ \\
\hline $204-$ & $6.63 \mathrm{E}-04$ & $7.19 E+00$ & $9.22 \mathrm{E}-05$ & $4.82 \mathrm{E}-0.3$ & $2.49 E+00$ & $1.94 \mathrm{E}-03$ & 4.07E-03 & $1.52 E+01$ & $2.68 \mathrm{E}-04$ & $1.14 \mathrm{E}-03$ & $2.14 E+00$ & 5.32E-04 \\
\hline 241-B & $3.41 \mathrm{E}-04$ & $9.45 \mathrm{E}+00$ & $3.61 \mathrm{E}-05$ & & $1.14 E+00$ & & $9.68 \mathrm{E}-04$ & $4.07 E+00$ & $2.38 \mathrm{E}-04$ & $2.41 \mathrm{E}-03$ & $2.30 \mathrm{E}+00$ & $1.05 \mathrm{E}-03$ \\
\hline 241-BX/BY & $1.86 \mathrm{E}-04$ & $8.61 E+00$ & $2.16 \mathrm{E}-05$ & & $6.90 \mathrm{E}+00$ & & $2.17 \mathrm{E}-03$ & $4.63 \mathrm{E}+00$ & $4.69 \mathrm{E}-04$ & $7.83 \mathrm{E}-04$ & $2.08 \mathrm{E}+00$ & $3.77 \mathrm{E}-04$ \\
\hline $241-\mathrm{C}$ & $1.08 \mathrm{E}-03$ & $4.06 \mathrm{E}+00$ & $2.65 E-04$ & $2.19 \mathrm{E}-03$ & $1.88 \mathrm{E}+00$ & $1.17 \mathrm{E}=03$ & & $7.65 E+00$ & & $3.71 \mathrm{E}-03$ & $1.02 \mathrm{E}+00$ & $3.64 \mathrm{E}-03$ \\
\hline 209-E & $1.03 \mathrm{E}-03$ & $1.76 \mathrm{E}+01$ & $5.86 \mathrm{E}-05$ & $3.74 \mathrm{E}-04$ & $5.06 \mathrm{E}+00$ & $7.40 \mathrm{E}-05$ & $8.40 \mathrm{E}-04$ & $1.30 \mathrm{E}+00$ & $6.46 \mathrm{E}-04$ & $4.45 \mathrm{E}-02$ & $8.54 \mathrm{E}+00$ & $5.21 \mathrm{E}-03$ \\
\hline & 8.13E-05 & $2.17 \mathrm{E}+00$ & $3.74 \mathrm{E}-05$ & 8.45E-04 & $1.48 \mathrm{E}+00$ & $5.71 E-04$ & & $1.66 \mathrm{E}+00$ & & $5.22 \mathrm{E}-03$ & $3.45 E+00$ & $1.51 \mathrm{E}-03$ \\
\hline & 7.87E-04 & $2.51 \mathrm{E}+00$ & $3.14 \mathrm{E}-04$ & $5.00 \mathrm{E}-04$ & $1.32 \mathrm{E}+00$ & $3.79 \mathrm{E}-04$ & 1. $10 \mathrm{E}-03$ & $2.91 E+00$ & $3.76 \mathrm{E}-04$ & 1. $65 \mathrm{E}-03$ & $4.81 E+00$ & 3.43E-04 \\
\hline $241-T$ & & $7.77 \mathrm{E}+00$ & & $6.48 \mathrm{E}-04$ & $2.34 \mathrm{E}+00$ & $2.77 \mathrm{E}-04$ & $2.99 \mathrm{E}-03$ & $6.03 \mathrm{E}-01$ & $4.97 \mathrm{E}-03$ & $6.57 \mathrm{E}-04$ & $6.28 \mathrm{E}+00$ & $1.05 \mathrm{E}-04$ \\
\hline 241-TX/TY & $5.02 \mathrm{E}-04$ & $5.47 \mathrm{E}-01$ & $9.18 \mathrm{E}-04$ & $6.39 \mathrm{E}-04$ & $1.48 \mathrm{E}+00$ & 4.32E-04 & & $4.58 \mathrm{E}-01$ & & $4.46 \mathrm{E}-04$ & $6.88 E+00$ & $6.49 \mathrm{E}-05$ \\
\hline 241-U & $1.97 \mathrm{E}-03$ & $8.80 \mathrm{E}+00$ & $2.24 \mathrm{E}-04$ & 4.69E-04 & $1.32 \mathrm{E}+00$ & $3.56 \mathrm{E}-04$ & & $2.01 \mathrm{E}+00$ & & $1.49 \mathrm{E}-02$ & $9.23 \mathrm{E}+00$ & $1.61 \mathrm{E}-03$ \\
\hline
\end{tabular}

\begin{tabular}{|c|c|c|c|}
\hline \multicolumn{4}{|c|}{ Ratio Using All Individual Áctivities } \\
\hline & U-235 & Cs-137 & Ratio \\
\hline Average & $3.67 \mathrm{E}-03$ & $\overline{4.34 \mathrm{E}+00}$ & $8.45 \mathrm{E}-04$ \\
\hline Std. Dev. & 7.32E-03 & $3.46 \mathrm{E}+00$ & $+/-\quad 4.76 \mathrm{E}-04$ \\
\hline $95 \%$ Conf & $1.93 \mathrm{E}-03$ & $8.68 \mathrm{E}-01$ & \\
\hline
\end{tabular}

(1) A blank indicates no activity detected 


\section{U-236 Ratio Calcs for LLW}

\begin{tabular}{|c|c|c|c|c|c|c|c|c|c|c|c|c|}
\hline \multirow[b]{2}{*}{ Farm/Area } & \multicolumn{3}{|c|}{ Sample Set \#4 } & \multicolumn{3}{|c|}{ Sample Set \#5 } & \multicolumn{3}{|c|}{ Sample Set $\# 6$} & \multicolumn{3}{|c|}{ Sample Set $\# 7$} \\
\hline & -236 & Cs- 137 & Ratio & $\mathrm{U}-236$ & Cs-137 & Ratio & U-236 & Cs-137 & Ratio & $\mathrm{U}-236$ & Cs- 137 & Ratio \\
\hline & & & .06 & 09E-04 & +00 & $6.74 \mathrm{E}-05$ & $1.74 \mathrm{E}-02$ & 4.05 & 4.24 & $1.40 \mathrm{E}-03$ & $5.07 \mathbf{E}+00$ & $2.75 \mathrm{E}-0$ \\
\hline & & & $E-05$ & $04 \mathrm{E}-04$ & $3.36 \mathrm{E}+00$ & $2.09 \mathrm{E}-04$ & $2.01 \mathrm{E}-03$ & $1.75 \mathrm{E}+00$ & $1.15 \mathrm{E}-03$ & $9.85 \mathrm{E}-03$ & $7.47 \mathbf{E}+00$ & $1.32 \mathrm{E}-03$ \\
\hline & & +00 & $\Xi-04$ & $8.68 \mathrm{E}-04$ & $8.74 \mathrm{E}-01$ & $9.93 \mathrm{E}-04$ & & & & $8.64 \mathrm{E}-03$ & $3.28 \mathrm{E}+00$ & $2.64 \mathrm{E}-03$ \\
\hline & -04 & +00 & E-05 & $2.30 \mathrm{E}-04$ & $2.70 \mathrm{E}+00$ & 8.53E-05 & $4.98 \mathrm{E}-03$ & $1.00 \mathrm{E}+00$ & $4.98 \mathrm{E}-03$ & $2.73 E-03$ & 1.60 & $1.71 \mathrm{E}-03$ \\
\hline & $00 \mathrm{E}-04$ & 3.25 & $2.46 \mathrm{E}-04$ & $1.25 \mathrm{E}-03$ & $<.18$ & & & & & $6.91 \mathrm{E}-03$ & +00 & $2.62 \mathrm{E}-03$ \\
\hline & $78 E-04$ & 9.65 & $E-05$ & -03 & $1.54 \mathrm{E}+00$ & & $4.96 \mathrm{E}-04$ & $9.99 \mathrm{E}-01$ & E-04 & $1.47 \mathrm{E}-03$ & 4.39 & $3.35 \mathrm{E}-04$ \\
\hline & -04 & 7.19 & $5.96 \mathrm{E}-05$ & $3.12 E-03$ & $2.49 \mathrm{E}+00$ & $1.25 \mathrm{E}-03$ & $2.63 \mathrm{E}-03$ & 1.52 & $1.73 \mathbf{E}-04$ & 7.37E-04 & 2.14 & $3.44 \mathrm{E}-04$ \\
\hline & $21 E-04$ & $9.45 \mathrm{E}+00$ & $2.34 \mathrm{E}-05$ & & $1.14 \mathrm{E}+00$ & & $6.26 \mathrm{E}-04$ & $4.07 \mathrm{E}+00$ & $1.54 \mathbf{E}-04$ & $1.56 \mathrm{E}-03$ & 2.30 & $6.78 \mathrm{E}-04$ \\
\hline & $20 \mathrm{E}-04$ & 8.61 & $1.40 \mathrm{E}-05$ & & $6.90 \mathrm{E}+00$ & & $1.41 \mathrm{E}-03$ & $4.63 E+00$ & $3.04 \mathrm{E}-04$ & $5.07 E-04$ & $2.08 \mathrm{~F}$ & $2.44 \mathrm{E}-04$ \\
\hline & E-04 & 4.06 & $1.72 \mathrm{E}-04$ & $1.42 \mathrm{E}-03$ & $1.88 \mathrm{E}+00$ & $7.54 \mathrm{E}-04$ & & $7.65 \mathrm{E}+00$ & & $2.40 \mathrm{E}-03$ & $1.02 E+00$ & $2.35 \mathrm{E}-03$ \\
\hline & 67E-04 & & $3.79 \mathrm{E}-05$ & $2.42 \mathrm{E}-04$ & $5.06 \mathrm{E}+00$ & $4.78 \mathrm{E}-05$ & $5.43 \mathrm{E}-04$ & $1.30 \mathrm{E}+00$ & $4.18 \mathrm{E}-04$ & $2.88 \mathrm{E}-02$ & $8.54 E+00$ & $3.37 \mathrm{E}-03$ \\
\hline & & & & $5.47 \mathrm{E}-04$ & $1.48 \mathrm{E}+00$ & $3.69 \mathrm{E}-04$ & & $1.66 \mathrm{E}+00$ & & $3.38 \mathrm{E}-03$ & $3.45 \mathrm{E}+00$ & $9.80 \mathrm{E}-04$ \\
\hline 241-SY & $5.09 \mathrm{E}-04$ & $2.51 \mathrm{E}+00$ & $2.03 \mathrm{E}-04$ & $3.24 \mathrm{E}-04$ & $1.32 \mathrm{E}+00$ & $2.45 \mathrm{E}-04$ & 7.09E-04 & $2.91 \mathrm{E}+00$ & $2.44 \mathrm{E}-04$ & $1.07 \mathrm{E}-03$ & $4.81 E+00$ & $2.22 \mathrm{E}-04$ \\
\hline $241-\mathrm{T}$ & & $7.77 E+00$ & & 4.19E-04 & $2.34 E+00$ & $1.79 E-04$ & $1.94 \mathrm{E}-03$ & $6.03 \mathrm{E}-01$ & $3.21 \mathrm{E}-03$ & 4.25E-04 & $6.28 \mathrm{E}+00$ & $6.77 \mathrm{E}-05$ \\
\hline & $3.25 \mathrm{E}-04$ & $5.47 \mathrm{E}-01$ & $5.94 \mathrm{E}-04$ & 4.13E-04 & $1.48 \mathrm{E}+00$ & $2.79 \mathrm{E}-04$ & & $4.58 \mathrm{E}-01$ & & $2.89 \mathrm{E}-04$ & $6.88 \mathrm{E}+00$ & $4.20 \mathrm{E}-05$ \\
\hline $241-U$ & $1.28 \mathrm{E}-03$ & $8.80 E+00$ & $1.45 \mathrm{E}-04$ & $3.04 \mathrm{E}-04$ & $1.32 \mathrm{E}+00$ & $2.30 \mathrm{E}-04$ & & $2.01 \mathrm{E}+00$ & & $9.64 \mathrm{E}-03$ & $9.23 \mathrm{E}+00$ & $1.04 \mathrm{E}-03$ \\
\hline
\end{tabular}

\begin{tabular}{|c|c|c|c|c|}
\hline \multicolumn{5}{|c|}{ Ratio Using All Individual Activities } \\
\hline & $\mathrm{U}-236$ & Cs-137 & & Ratio \\
\hline Average & $2.37 \mathrm{E}-03$ & $4.34 \mathrm{E}+00$ & & $5.46 \mathrm{E}-04$ \\
\hline Std. Dev. & 4.73E-03 & $3.46 \mathrm{E}+00$ & $+/-$ & $3.08 \mathrm{E}-04$ \\
\hline $95 \%$ Conf & $1.25 \mathrm{E}-03$ & $8.68 \mathrm{E}-01$ & & \\
\hline
\end{tabular}

(1) A biank indicates no activity detected 


\section{U-238 Ratio Calcs for LLW}

\begin{tabular}{|c|c|c|c|c|c|c|c|c|c|c|c|c|}
\hline \multirow[b]{2}{*}{ Farm/Area } & \multicolumn{3}{|c|}{ Sample Set \#4 } & \multicolumn{3}{|c|}{ Sample Set \#5 } & \multicolumn{3}{|c|}{ Sample Set \#6 } & \multicolumn{3}{|c|}{ Sample Set $\# 7$} \\
\hline & $\mathrm{J}-238$ & Cs- 137 & Ratio & U-238 & Cs- 137 & Ratio & $\mathrm{U}-238$ & Cs- 137 & Ratio & U-238 & Cs-137 & Ratio \\
\hline $241=$ & $.49 \mathrm{E}-03$ & $.51 \mathrm{E}+00$ & 04 & $74 \mathrm{E}-03$ & $4.59 \mathrm{E}+00$ & $:-03$ & $4.90 \mathrm{E}-01$ & $4.09 \mathrm{E}+00$ & $1.20 \mathrm{E}-0 \mathrm{l}$ & $3.94 \mathrm{E}-02$ & $5.07 \mathrm{E}+00$ & $7.77 \mathrm{E}-03$ \\
\hline $241-$ & $2.69 \mathrm{E}-03$ & $6.49 \mathrm{E}+00$ & 4. $15 \mathrm{E}-04$ & $1.99 \mathrm{E}-02$ & $3.36 \mathrm{E}+00$ & $5.91 \mathrm{E}-03$ & $5.68 \mathrm{E}-02$ & $1.75 \mathrm{E}+00$ & $3.25 E-02$ & $2.78 \mathrm{E}-01$ & $7.47 \mathrm{E}+00$ & $3.72 \mathrm{E}-02$ \\
\hline & $3.16 \mathrm{E}-02$ & $4.83 E+00$ & $6.54 \mathrm{E}-03$ & $2.45 \mathrm{E}-02$ & $8.74 \mathrm{E}-01$ & $2.80 \mathrm{E}-02$ & & & & $2.44 \mathrm{E}-0 \mathrm{t}$ & $\$+00$ & $7.44 \mathrm{E}-02$ \\
\hline & $47 \mathrm{E}-03$ & $7.36 \mathrm{E}+00$ & $4.71 \mathrm{E}-04$ & $.50 \mathrm{E}-03$ & $2.70 \mathrm{E}+00$ & $2.41 \mathrm{E}-03$ & $1.41 \mathrm{E}-01$ & $1.00 \mathrm{E}+00$ & $1.41 \mathrm{E}-01$ & $7.71 \mathrm{E}-02$ & & $2 \mathrm{E}-02$ \\
\hline & $26 \mathrm{E}-02$ & $3.25 \mathrm{E}+00$ & $6.94 \mathrm{E}-03$ & $.53 \mathrm{E}-02$ & $<.18$ & & & & & $1.95 \mathrm{E}-01$ & $2.64 \mathrm{E}+00$ & $7.39 \mathrm{E}-02$ \\
\hline & 7.83 & $9.65 \mathrm{E}+00$ & $8.12 \mathrm{E}-04$ & $88 \mathrm{E}-02$ & $1.54 \mathrm{E}+00$ & $1.87 \mathrm{E}-02$ & $1.40 \mathrm{E}-02$ & $9.99 \mathrm{E}-01$ & $1.40 \mathrm{E}-02$ & $4.15 \mathrm{E}-02$ & $4.39 E+00$ & $9.45 \mathrm{E}-03$ \\
\hline & 1.21 & 7.191 & & $8.80 \mathrm{E}-02$ & $2.49 \mathrm{E}+00$ & $3.53 \mathrm{E}-02$ & $7.43 \mathrm{E}-02$ & $E+01$ & $4.89 \mathrm{E}-03$ & $2.08 \mathrm{E}-02$ & +00 & $9.72 \mathrm{E}-03$ \\
\hline & $8-03$ & $9.45 \mathrm{E}$ & & & $1.14 \mathrm{E}+00$ & & $1.77 \mathrm{E}-02$ & $E+00$ & $4.34 \mathrm{E}-03$ & $4.40 \mathrm{E}-02$ & $E+00$ & $E-02$ \\
\hline $\mathrm{BX} / \mathrm{BY}$ & $3.40 \mathrm{E}-03$ & $8.61 E$ & & & $6.90 \mathrm{E}+00$ & & $3.97 \mathrm{E}-02$ & $4.63 \mathrm{E}+00$ & 8.57E-03 & $1.43 \mathrm{E}-02$ & $2.08 \mathrm{E}+00$ & $E-03$ \\
\hline & $1.97 \mathrm{E}-02$ & $4.06 \mathrm{E}+00$ & $4.84 \mathrm{E}-03$ & $1.00 \mathrm{E}-02$ & $1.88 \mathrm{E}+00$ & $2.13 \mathrm{E}-02$ & & $7.65 \mathrm{E}+00$ & & $6.77 \mathrm{E}-02$ & $1.02 E+00$ & $4 E-02$ \\
\hline $09-\mathrm{F}$ & $1.88 \mathrm{E}-02$ & $1.76 \mathrm{E}+01$ & $1.07 \mathrm{E}-03$ & $6.83 \mathrm{E}-03$ & $5.06 \mathrm{E}+00$ & $1.35 \mathrm{E}-03$ & $1.53 \mathrm{E}-02$ & $1.30 E+00$ & $1.18 \mathrm{E}-02$ & 8.13E-01 & $8.54 E+00$ & $9.52 \mathrm{E}-02$ \\
\hline & $1.48 \mathrm{E}-03$ & $2.17 \mathrm{E}+00$ & $6.84 \mathrm{E}-04$ & $1.54 \mathrm{E}-02$ & $1.48 \mathrm{E}+00$ & $1.04 \mathrm{E}-02$ & & $1.66 \mathrm{E}+00$ & & $9.54 \mathrm{E}-02$ & $3.45 \mathrm{E}+00$ & $2.77 \mathrm{E}-02$ \\
\hline & $1.44 \mathrm{E}-02$ & $2.51 \mathrm{E}+00$ & $5.72 \mathrm{E}-03$ & $9.14 \mathrm{E}-03$ & $1.32 \mathrm{E}+00$ & $6.92 \mathrm{E}-03$ & $2.00 \mathrm{E}-02$ & $2.91 \mathrm{E}+00$ & $6.87 \mathrm{E}-03$ & $3.01 \mathrm{E}-02$ & $4.81 \mathrm{E}+00$ & $6.26 \mathrm{E}-03$ \\
\hline & & $7.77 \mathrm{E}+00$ & & 1. $18 \mathrm{E}-02$ & $2.34 \mathrm{E}+00$ & $5.06 \mathrm{E}-03$ & $5.47 \mathrm{E}-02$ & $6.03 \mathrm{E}-01$ & $9.07 \mathrm{E}-02$ & $1.20 \mathrm{E}-02$ & $6.28 \mathrm{E}+00$ & $1.91 \mathrm{E}-03$ \\
\hline & $9.17 \mathrm{E}-03$ & $5.47 \mathrm{E}-01$ & & $1.17 \mathrm{E}-02$ & $1.48 \mathrm{E}+00$ & $7.88 \mathrm{E}-03$ & & $4.58 \mathrm{E}-01$ & & $8.15 \mathrm{E}-03$ & $6.88 \mathrm{E}+00$ & $1.18 \mathrm{E}-03$ \\
\hline $241-0$ & $3.60 \mathrm{E}-02$ & $8.80 \mathrm{E}+00$ & 4.09E-03 & $8.57 \mathrm{E}-03$ & $1.32 E+00$ & $6.49 \mathrm{E}-03$ & & $2.01 \mathrm{E}+00$ & & $2.72 \mathrm{E}-01$ & $9.23 E+00$ & $2.95 \mathrm{E}-02$ \\
\hline
\end{tabular}

\begin{tabular}{|c|c|c|c|}
\hline \multicolumn{4}{|c|}{ Ratio Using All Individual Activities } \\
\hline & U-238 & Cs-137 & Ratio \\
\hline Average & $6.69 \mathrm{E}-02$ & $4.34 \mathrm{E}+00$ & $1.54 \mathrm{E}-02$ \\
\hline Std. Dev. & $1.34 \mathrm{E}-01$ & $3.46 \mathrm{E}+00$ & $+/-8.70 \mathrm{E}-03$ \\
\hline $95 \%$ Conf & $3.53 \mathrm{E}-02$ & 8.68E-01 & \\
\hline
\end{tabular}

(1) A blank indicates no activity detected 


\section{Np-237 Ratio Calcs for $L L W$}

\begin{tabular}{l|ccc|ccc} 
& \multicolumn{3}{|c|}{ Sample Set \#6 } & \multicolumn{3}{c}{ Sample Set \#7 } \\
Farm/Area & Np-237 & Cs-137 & Ratio & Np-237 & Cs-137 & Ratio \\
\hline \hline & & & & & & \\
241-AN & $<6.30 \mathrm{E}-02$ & $4.09 \mathrm{E}+00$ & & $<2.90 \mathrm{E}-02$ & $5.07 \mathrm{E}+00$ \\
241-AP & $<2.60 \mathrm{E}-02$ & $1.75 \mathrm{E}+00$ & & $5.10 \mathrm{E}-02$ & $7.47 \mathrm{E}+00$ & $6.83 \mathrm{E}-03$ \\
$241-\mathrm{AW}$ & & & & $3.00 \mathrm{E}-02$ & $3.28 \mathrm{E}+00$ & $9.15 \mathrm{E}-03$ \\
$241-\mathrm{AY}$ & $4.30 \mathrm{E}-02$ & $1.00 \mathrm{E}+00$ & $4.30 \mathrm{E}-02$ & $3.20 \mathrm{E}-02$ & $1.60 \mathrm{E}+00$ & $2.00 \mathrm{E}-02$ \\
244-A & & & & $<1.30 \mathrm{E}-01$ & $2.64 \mathrm{E}+00$ & \\
242-A & $<3.60 \mathrm{E}-02$ & $9.99 \mathrm{E}-01$ & & $<3.10 \mathrm{E}-02$ & $4.39 \mathrm{E}+00$ & \\
204-AR & $<6.10 \mathrm{E}-02$ & $1.52 \mathrm{E}+01$ & & $<5.90 \mathrm{E}-02$ & $2.14 \mathrm{E}+00$ & \\
$241-\mathrm{B}$ & $<7.50 \mathrm{E}-02$ & $4.07 \mathrm{E}+00$ & & $1.90 \mathrm{E}-01$ & $2.30 \mathrm{E}+00$ & $8.26 \mathrm{E}-02$ \\
$241-\mathrm{BX} / \mathrm{BY}$ & $2.90 \mathrm{E}-02$ & $4.63 \mathrm{E}+00$ & $6.26 \mathrm{E}-03$ & $3.40 \mathrm{E}-02$ & $2.08 \mathrm{E}+00$ & $1.63 \mathrm{E}-02$ \\
$241-\mathrm{C}$ & $<2.90 \mathrm{E}-02$ & $7.65 \mathrm{E}+00$ & & $<9.20 \mathrm{E}-02$ & $1.02 \mathrm{E}+00$ & \\
209-E & $<6.40 \mathrm{E}-02$ & $1.30 \mathrm{E}+00$ & & $3.70 \mathrm{E}-02$ & $8.54 \mathrm{E}+00$ & $4.33 \mathrm{E}-03$ \\
$241-\mathrm{S} / \mathrm{SX}$ & $2.50 \mathrm{E}-02$ & $1.66 \mathrm{E}+00$ & $1.51 \mathrm{E}-02$ & $<8.00 \mathrm{E}-02$ & $3.45 \mathrm{E}+00$ & \\
$241-\mathrm{SY}$ & $<9.40 \mathrm{E}-02$ & $2.91 \mathrm{E}+00$ & & $<1.10 \mathrm{E}-02$ & $4.81 \mathrm{E}+00$ & \\
$241-\mathrm{T}$ & $<5.90 \mathrm{E}-02$ & $6.03 \mathrm{E}-01$ & & $<5.00 \mathrm{E}-02$ & $6.28 \mathrm{E}+00$ & \\
$241-\mathrm{TX} / \mathrm{TY}$ & $<2.10 \mathrm{E}-02$ & $4.58 \mathrm{E}-01$ & & $2.30 \mathrm{E}-02$ & $6.88 \mathrm{E}+00$ & $3.34 \mathrm{E}-03$ \\
$241-\mathrm{U}$ & $<6.80 \mathrm{E}-02$ & $2.01 \mathrm{E}+00$ & & $1.70 \mathrm{E}-02$ & $9.23 \mathrm{E}+00$ & $1.84 \mathrm{E}-03$
\end{tabular}

\begin{tabular}{|c|c|c|c|c|}
\hline \multicolumn{5}{|c|}{ Ratio Using All Individual Activities } \\
\hline & U-238 & Cs-137 & & Ratio \\
\hline Average & $4.65 \mathrm{E}-02$ & $3.98 \mathrm{E}+00$ & & $\overline{1.17 \mathrm{E}-02}$ \\
\hline Std. Dev. & 4.63E-02 & $3.20 \mathrm{E}+00$ & $+1-$ & $7.64 \mathrm{E}-03$ \\
\hline $95 \%$ Conf & $2.73 \mathrm{E}-02$ & $1.14 \mathrm{E}+00$ & & \\
\hline
\end{tabular}

(1) A blank indicates no activity detected 


\section{Pu-238 Ratio Calcs for LLW}

\begin{tabular}{|c|c|c|c|c|c|c|c|c|c|c|c|c|c|c|c|}
\hline \multirow[b]{2}{*}{ Farm/Area } & \multicolumn{3}{|c|}{ Sample Set \#3 } & \multicolumn{3}{|c|}{ Sample Set $\# 4$} & \multicolumn{3}{|c|}{ Sample Set \#5 } & \multicolumn{3}{|c|}{ Sample Set \#6 } & \multicolumn{3}{|c|}{ Sample Set $\# 7$} \\
\hline & Pu-238 & Cs-137 & Ratio & Pu-238 & Cs- 137 & Ratio & Pu-238 & Cs-137 & Ratio & Pu-238 & Cs-137 & Ratio & Pu-238 & Cs-137 & Ratio \\
\hline 241-AN & & $5.88 \mathrm{E}-01$ & & & $6.51 \mathrm{E}+00$ & & & $4.59 \mathrm{E}+00$ & & 4.91E-02 & $4.09 \mathrm{E}+00$ & 1.20E-02 & $3.63 \mathrm{E}-02$ & $5.07 \mathbf{E}+00$ & $7.16 \mathrm{E}-03$ \\
\hline 241-AP & & $1.73 E+00$ & & & $6.49 \mathrm{E}+00$ & & & $3.36 \mathrm{E}+00$ & & $1.56 \mathrm{E}-02$ & $1.75 \mathbf{E}+00$ & $8.90 \mathrm{E}-03$ & $2.45 \mathrm{E}-01$ & $7.47 \mathrm{E}+00$ & $3.29 \mathrm{E}-02$ \\
\hline 241-AW & & $2.07 E+00$ & & & $4.83 \mathrm{E}+00$ & & & $8.74 \mathrm{E}-01$ & & & & & $2.94 \mathrm{E}-02$ & $3.28 \mathrm{E}+00$ & $8.98 E-03$ \\
\hline 241-AY & & $3.30 \mathrm{E}-01$ & & & $7.36 \mathrm{E}+00$ & & & $2.70 \mathrm{E}+00$ & & $5.74 \mathrm{E}-02$ & $1.00 \mathrm{E}+00$ & $5.74 \mathrm{E}-02$ & $3.09 \mathrm{E}-02$ & $1.60 \mathrm{E}+00$ & $1.93 \mathrm{E}-02$ \\
\hline 244-A & & $6.09 \mathrm{E}-01$ & & & $3.25 \mathrm{E}+00$ & & & $<.18$ & & & & & $1.22 \mathrm{E}-01$ & $2.64 \mathrm{E}+00$ & $4.62 \mathrm{E}-02$ \\
\hline $242-A$ & & $4.20 \mathrm{E}+00$ & & & $9.65 \mathrm{E}+00$ & & & $1.54 \mathrm{E}+00$ & & 4.12E-03 & $9.99 \mathrm{E}-01$ & 4.13E-03 & $6.63 \mathrm{E}-02$ & $4.39 \mathrm{E}+00$ & $1.51 \mathrm{E}-02$ \\
\hline 204-AR & $5.18 \mathrm{E}-02$ & $7.77 \mathrm{E}+00$ & $6.66 \mathrm{E}-03$ & & $7.19 \mathrm{E}+00$ & & & $2.49 \mathrm{E}+00$ & & $4.83 \mathrm{E}-02$ & $1.52 \mathrm{E}+01$ & $3.18 \mathrm{E}-03$ & $4.86 \mathrm{E}-01$ & $2.14 \mathrm{E}+00$ & $2.27 \mathrm{E}-01$ \\
\hline 241-B & & $1.12 \mathrm{E}+01$ & & & $9.45 \mathrm{E}+00$ & & & $1.14 \mathrm{E}+00$ & & $2.60 \mathrm{E}-02$ & $4.07 \mathrm{E}+00$ & $6.39 \mathrm{E}-03$ & $1.54 \mathrm{E}-02$ & $2.30 \mathrm{E}+00$ & $6.70 \mathrm{E}-03$ \\
\hline 241-BX/BY & & $1.37 \mathrm{E}+01$ & & & $8.61 E+00$ & & & $6.90 \mathrm{E}+00$ & & $3.31 \mathrm{E}-03$ & $4.63 \mathrm{E}+00$ & $7.16 \mathrm{E}-04$ & $8.44 \mathrm{E}-03$ & $2.08 \mathrm{E}+00$ & $4.06 \mathrm{E}-03$ \\
\hline $241-C$ & & $5.45 \mathrm{E}+01$ & & & $4.06 \mathrm{E}+00$ & & & $1.88 \mathrm{E}+00$ & & $7.93 \mathrm{E}-02$ & $7.65 \mathrm{E}+00$ & $1.04 \mathrm{E}-02$ & $5.62 \mathrm{E}-03$ & $1.02 \mathrm{E}+00$ & $5.51 \mathrm{E}-03$ \\
\hline 209-E & & $2.84 \mathrm{E}+00$ & & & $1.76 \mathrm{E}+01$ & & & $5.06 \mathrm{E}+00$ & & & $1.30 \mathrm{E}+00$ & & $6.55 \mathrm{E}-02$ & $8.54 \mathrm{E}+00$ & $7.67 \mathrm{E}-03$ \\
\hline 241-S/SX & & $9.11 \mathrm{E}-01$ & & & $2.17 \mathrm{E}+00$ & & $1.14 \mathrm{E}-01$ & $1.48 \mathrm{E}+00$ & $7.69 \mathrm{E}-02$ & $9.91 \mathrm{E}-03$ & $1.66 \mathrm{E}+00$ & $5.97 \mathrm{E}-03$ & $9.08 \mathrm{E}-02$ & $3.45 \mathrm{E}+00$ & $2.63 \mathrm{E}-02$ \\
\hline 241-SY & & $3.25 \mathrm{E}+00$ & & & $2.51 \mathrm{E}+00$ & & & $1.32 \mathrm{E}+00$ & & $1.90 \mathrm{E}-02$ & $2.91 E+00$ & $6.52 \mathrm{E}-03$ & 1.37E-02 & $4.81 \mathrm{E}+00$ & $2.86 \mathrm{E}-03$ \\
\hline $241-T$ & & $1.41 \mathrm{E}+00$ & & $1.77 \mathrm{E}+00$ & $7.77 \mathrm{E}+00$ & $2.28 \mathrm{E}-01$ & & $2.34 \mathrm{E}+00$ & & 4.39E-03 & $6.03 \mathrm{E}-01$ & $7.28 \mathrm{E}-03$ & $1.90 \mathrm{E}-02$ & $6.28 \mathrm{E}+00$ & $3.03 \mathrm{E}-03$ \\
\hline 241-TX/TY & & $6.19 \mathrm{E}+00$ & & & 5.47E-01 & & & $1.48 \mathrm{E}+00$ & & & $4.58 \mathrm{E}-01$ & & $2.16 \mathrm{E}-03$ & $6.88 \mathrm{E}+00$ & $3.15 \mathrm{E}-04$ \\
\hline $241-U$ & & $2.66 \mathrm{E}+00$ & & & $8.80 \mathrm{E}+00$ & & & $1.32 \mathrm{E}+00$ & & $1.77 \mathrm{E}-02$ & $2.01 \mathrm{E}+00$ & $8.83 \mathrm{E}-03$ & $2.97 \mathrm{E}-02$ & $9.23 \mathrm{E}+00$ & $3.22 \mathrm{E}-03$ \\
\hline
\end{tabular}

\begin{tabular}{|lccrr|}
\hline Ratio Using All Individual Activities & & \\
& Pu-238 & Cs-137 & & Ratio \\
\cline { 2 - 3 } Average & 0.11 & 4.92 & & 0.023 \\
Std. Dev. & 0.32 & 6.70 & $+/-$ & 0.024 \\
95\% Conf. & 0.11 & 1.50 & & \\
\hline
\end{tabular}




\section{Pu-239 Ratio Cales for LLW}

\begin{tabular}{|c|c|c|c|c|c|c|c|c|c|c|c|c|c|c|c|}
\hline \multirow[b]{2}{*}{ Farm/Area } & \multicolumn{3}{|c|}{ Sample Set \#3 } & \multicolumn{3}{|c|}{ Sample Set \#4 } & \multicolumn{3}{|c|}{ Sample Set \#5 } & \multicolumn{3}{|c|}{ Sample Set \#6 } & \multicolumn{3}{|c|}{ Sample Set \#7 } \\
\hline & Pu-239 & Cs-137 & Ratio & $\mathrm{Pu}-239$ & Cs-137 & Ratio & Pu-239 & Cs-137 & Ratio & Pu-239 & Cs-137 & Ratio & $\mathrm{Pu}-239$ & $\mathrm{Cs}-137$ & Ratio \\
\hline 241-AN & & $5.88 \mathrm{E}-01$ & & & $6.51 \mathrm{E}+00$ & & & $4.59 \mathrm{E}+00$ & & $1.26 \mathrm{E}-01$ & $4.09 \mathrm{E}+00$ & $3.07 \mathrm{E}-02$ & $9.29 \mathrm{E}-02$ & $5.07 \mathrm{E}+00$ & $1.83 \mathrm{E}-02$ \\
\hline 241-AP & & $1.73 \mathrm{E}+00$ & & & $6.49 \mathrm{E}+00$ & & & $3.36 \mathrm{E}+00$ & & $3.99 \mathrm{E}-02$ & $1.75 \mathbf{E}+00$ & $2.28 \mathrm{E}-02$ & $6.28 \mathrm{E}-01$ & $7.47 \mathrm{E}+00$ & $8.40 \mathrm{E}-02$ \\
\hline 241-AW & & $2.07 \mathrm{E}+00$ & & & $4.83 \mathrm{E}+00$ & & & $8.74 \mathrm{E}-01$ & & & & & $7.53 \mathrm{E}-02$ & $3.28 \mathrm{E}+00$ & $2.30 \mathrm{E}-02$ \\
\hline 241-AY & & $3.30 \mathrm{E}-01$ & & & $7.36 \mathrm{E}+00$ & & & $2.70 \mathrm{E}+00$ & & $1.47 \mathrm{E}-01$ & $1.00 \mathrm{E}+00$ & $1.47 \mathrm{E}-01$ & $7.91 \mathrm{E}-02$ & $1.60 \mathrm{E}+00$ & $4.94 \mathrm{E}-02$ \\
\hline 244-A & & $6.09 \mathrm{E}-01$ & & & $3.25 \mathrm{E}+00$ & & & $<.18$ & & & & & $3.12 \mathrm{E}-01$ & $2.64 \mathrm{E}+00$ & $1.18 \mathrm{E}-01$ \\
\hline 242-A & & $4.20 \mathrm{E}+00$ & & & $9.65 \mathrm{E}+00$ & & & $1.54 \mathrm{E}+00$ & & $1.05 \mathrm{E}-02$ & $9.99 \mathrm{E}-01$ & $1.06 \mathrm{E}-02$ & $1.70 \mathrm{E}-01$ & $4.39 E+00$ & $3.86 \mathrm{E}-02$ \\
\hline 204-AR & $1.32 \mathrm{E}-01$ & $7.77 \mathrm{E}+00$ & $1.70 \mathrm{E}-02$ & & $7.19 \mathrm{E}+00$ & & & $2.49 \mathrm{E}+00$ & & $1.24 \mathrm{E}-01$ & $1.52 \mathrm{E}+01$ & $8.14 E-03$ & $1.24 \mathrm{E}+00$ & $2.14 \mathrm{E}+00$ & $5.81 \mathrm{E}-01$ \\
\hline $241-B$ & & $1.12 E+01$ & & & $9.45 \mathrm{E}+00$ & & & $1.14 \mathrm{E}+00$ & & $6.65 \mathrm{E}-02$ & $4.07 \mathrm{E}+00$ & $1.64 \mathrm{E}-02$ & $3.94 \mathrm{E}-02$ & $2.30 \mathrm{E}+00$ & $1.71 \mathrm{E}-02$ \\
\hline 241-BX/BY & & $1.37 \mathrm{E}+01$ & & & $8.61 E+00$ & & & $6.90 \mathrm{E}+00$ & & $8.48 \mathrm{E}-03$ & $4.63 \mathrm{E}+00$ & $1.83 E-03$ & $2.16 \mathrm{E}-02$ & $2.08 \mathrm{E}+00$ & $1.04 \mathrm{E}-02$ \\
\hline $241-C$ & & $5.45 \mathrm{E}+01$ & & & $4.06 E+00$ & & & $1.88 \mathrm{E}+00$ & & $2.03 \mathrm{E}-01$ & $7.65 \mathrm{E}+00$ & $2.65 \mathrm{E}-02$ & $1.44 \mathrm{E}-02$ & $1.02 \mathrm{E}+00$ & $1.41 \mathrm{E}-02$ \\
\hline 209-E & & $2.84 \mathrm{E}+00$ & & & $1.76 \mathrm{E}+01$ & & & $5.06 \mathrm{E}+00$ & & & $1.30 E+00$ & & $1.68 \mathrm{E}-01$ & $8.54 \mathrm{E}+00$ & $1.96 \mathrm{E}-02$ \\
\hline 241-S/SX & & $9.11 \mathrm{E}-01$ & & & $2.17 \mathrm{E}+00$ & & $2.91 \mathrm{E}-01$ & $1.48 \mathrm{E}+00$ & $1.97 \mathrm{E}-01$ & $2.54 \mathrm{E}-02$ & $1.66 \mathrm{E}+00$ & $1.53 \mathrm{E}-02$ & $2.32 \mathrm{E}-01$ & $3.45 \mathrm{E}+00$ & $6.73 \mathrm{E}-02$ \\
\hline $241-S Y$ & & $3.25 \mathrm{E}+00$ & & & $2.51 \mathrm{E}+00$ & & & $1.32 \mathrm{E}+00$ & & $4.85 \mathrm{E}-02$ & $2.91 \mathrm{E}+00$ & $1.67 \mathrm{E}-02$ & $3.52 \mathrm{E}-02$ & $4.81 \mathrm{E}+00$ & $7.31 \mathrm{E}-03$ \\
\hline $241-\mathrm{T}$ & & $1.41 E+00$ & & $4.53 \mathrm{E}+00$ & $7.77 \mathrm{E}+00$ & $5.83 \mathrm{E}-0$ & & $2.34 E+00$ & & $1.12 \mathrm{E}-02$ & $6.03 E-01$ & $1.86 \mathrm{E}-02$ & $4.87 \mathrm{E}-02$ & $6.28 \mathrm{E}+00$ & $7.75 \mathrm{E}-03$ \\
\hline $241-\mathrm{TX} / \mathrm{TY}$ & & $6.19 \mathrm{E}+00$ & & & $5.47 \mathrm{E}-01$ & & & $1.48 \mathrm{E}+00$ & & & $4.58 \mathrm{E}-01$ & & $5.54 \mathrm{E}-03$ & $6.88 \mathrm{E}+00$ & $8.05 E-04$ \\
\hline $241-U$ & & $2.66 \mathrm{E}+00$ & & & $8.80 \mathrm{E}+00$ & & & $1.32 \mathrm{E}+00$ & & $4.54 \mathrm{E}-02$ & $2.01 \mathrm{E}+00$ & $2.26 \mathrm{E}-02$ & $7.60 \mathrm{E}-02$ & $9.23 \mathrm{E}+00$ & $8.23 \mathrm{E}-03$ \\
\hline
\end{tabular}

(1) A blank indicates no activity detected

\begin{tabular}{|lccrr|}
\hline Ratio Using All Individual Activities & & \\
& Pu-239 & Cs-137 & & Ratio \\
\cline { 2 - 3 } Average & 0.29 & 4.92 & & 0.059 \\
Std. Dev. & 0.81 & 6.70 & $+1-$ & 0.061 \\
$95 \%$ Conf. & 0.28 & 1.50 & & \\
\hline
\end{tabular}


Pu-240 Ratio Calcs for LLW

\begin{tabular}{|c|c|c|c|c|c|c|c|c|c|c|c|c|c|c|c|}
\hline \multirow[b]{2}{*}{ Farm/Area } & \multicolumn{3}{|c|}{ Sample Set \#3 } & \multicolumn{3}{|c|}{ Sample Set \#4 } & \multicolumn{3}{|c|}{ Sample Set \#5 } & \multicolumn{3}{|c|}{ Sample Set $\# 6$} & \multicolumn{3}{|c|}{ Sample Set \#7 } \\
\hline & Pu-240 & Cs- 137 & Ratio & Pu-240 & Cs- 137 & Ratio & $\mathrm{Pu}-240$ & Cs- 137 & Ratio & Pu-240 & Cs-137 & Ratio & $\mathrm{Pu}-240$ & Cs-137 & Ratio \\
\hline 241-AN & & $5.88 \mathrm{E}-01$ & & & $6.51 \mathrm{E}+00$ & & & $4.59 \mathrm{E}+00$ & & $7.45 \mathrm{E}-02$ & $4.09 \mathrm{E}+00$ & $1.82 \mathrm{E}-02$ & $5.51 \mathrm{E}-02$ & $5.07 \mathrm{E}+00$ & $1.09 \mathrm{E}-02$ \\
\hline 241-AP & & $1.73 E+00$ & & & $6.49 \mathrm{E}+00$ & & & $3.36 \mathrm{E}+00$ & & $2.36 \mathrm{E}-02$ & $1.75 \mathrm{E}+00$ & $1.35 \mathrm{E}-02$ & $3.72 \mathrm{E}-01$ & $7.47 \mathrm{E}+00$ & $4.99 \mathrm{E}-02$ \\
\hline $241-\mathrm{AW}$ & & $2.07 \mathrm{E}+00$ & & & $4.83 E+00$ & & & $8.74 \mathrm{E}-01$ & & & & & 4.47E-02 & $3.28 \mathrm{E}+00$ & $1.36 \mathrm{E}-02$ \\
\hline 241-AY & & $3.30 \mathrm{E}-01$ & & & $7.36 \mathrm{E}+00$ & & & $2.70 \mathrm{E}+00$ & & 8.71E-02 & $1.00 \mathrm{E}+00$ & $8.71 \mathrm{E}-02$ & 4.69E- 02 & $1.60 \mathrm{E}+00$ & 2.93E-02 \\
\hline $244-A$ & & $6.09 \mathrm{E}-01$ & & & $3.25 \mathrm{E}+00$ & & & $<.18$ & & & & & 1.85E-01 & $2.64 \mathrm{E}+00$ & $7.01 \mathrm{E}-02$ \\
\hline $242-A$ & & $4.20 E+00$ & & & $9.65 \mathrm{E}+00$ & & & $1.54 \mathrm{E}+00$ & & $6.26 \mathrm{E}-03$ & $9.99 \mathrm{E}-01$ & $6.26 \mathrm{E}-03$ & $1.01 \mathrm{E}-0 \mathrm{l}$ & $4.39 \mathrm{E}+00$ & $2.29 \mathrm{E}-02$ \\
\hline 204-AR & $7.86 \mathrm{E}-02$ & $7.77 \mathrm{E}+00$ & $1.01 \mathrm{E}-02$ & & $7.19 E+00$ & & & $2.49 \mathrm{E}+00$ & & $7.34 \mathrm{E}-02$ & $1.52 \mathrm{E}+01$ & $4.83 \mathrm{E}-03$ & $7.37 \mathrm{E}-01$ & $2.14 E+00$ & $3.45 \mathrm{E}-01$ \\
\hline 241-B & & $1.12 \mathrm{E}+01$ & & & $9.45 \mathrm{E}+00$ & & & $1.14 \mathrm{E}+00$ & & $3.95 \mathrm{E}-02$ & $4.07 \mathrm{E}+00$ & $9.70 \mathrm{E}-03$ & $2.34 \mathrm{E}-02$ & $2.30 \mathrm{E}+00$ & $1.02 \mathrm{E}-02$ \\
\hline $41-B X / B Y$ & & $1.37 \mathrm{E}+01$ & & & $8.61 \mathrm{E}+00$ & & & $6.90 \mathrm{E}+00$ & & $5.03 \mathrm{E}-03$ & $4.63 \mathrm{E}+00$ & $1.09 \mathrm{E}-03$ & $1.28 \mathrm{E}-02$ & $2.08 \mathrm{E}+00$ & $6.16 \mathrm{E}-03$ \\
\hline $241-C$ & & $5.45 \mathrm{E}+01$ & & & $4.06 \mathrm{E}+00$ & & & $1.88 \mathrm{E}+00$ & & $1.20 \mathrm{E}-01$ & $7.65 \mathrm{E}+00$ & $1.57 \mathrm{E}-02$ & 8.53E-03 & $1.02 \mathrm{E}+00$ & 8.36E-03 \\
\hline 209-E & & $2.84 \mathrm{E}+00$ & & & $1.76 \mathrm{E}+01$ & & & $5.06 \mathrm{E}+00$ & & & $1.30 \mathrm{E}+00$ & & $9.94 \mathrm{E}-02$ & $8.54 E+00$ & $1.16 \mathrm{E}-02$ \\
\hline 241-S/SX & & $9.11 \mathrm{E}-01$ & & & $2.17 \mathrm{E}+00$ & & $1.73 \mathrm{E}-01$ & $1.48 \mathrm{E}+00$ & $1.17 \mathrm{E}-01$ & $1.50 \mathrm{E}-02$ & $1.66 \mathrm{E}+00$ & $9.06 \mathrm{E}-03$ & $1.38 \mathrm{E}-01$ & $3.45 \mathrm{E}$ & $3.99 \mathrm{E}-02$ \\
\hline 41-SY & & $3.25 \mathrm{E}+00$ & & & $2.51 \mathrm{E}+00$ & & & $1.32 \mathrm{E}+00$ & & $2.88 \mathrm{E}-02$ & $2.91 E+00$ & $9.89 \mathrm{E}-03$ & $2.09 \mathrm{E}-02$ & $4.81 \mathrm{E}+00$ & $4.34 \mathrm{E}-03$ \\
\hline $41-T$ & & $1.41 \mathrm{E}+00$ & & $2.69 \mathrm{E}+00$ & $7.77 \mathrm{E}+00$ & $3.46 \mathrm{E}-01$ & & $2.34 \mathrm{E}+00$ & & 6.67E-03 & $6.03 \mathrm{E}-01$ & $1.11 \mathrm{E}-02$ & $2.89 \mathrm{E}-02$ & $6.28 \mathrm{E}+00$ & $4.60 \mathrm{E}-03$ \\
\hline 41-TX/TY & & $6.19 E+00$ & & & $5.47 \mathrm{E}-01$ & & & $1.48 \mathrm{E}+00$ & & & $4.58 \mathrm{E}-01$ & & $3.28 \mathrm{E}-03$ & $6.88 \mathrm{E}+00$ & $4.77 \mathrm{E}-04$ \\
\hline $241-\mathrm{U}$ & & $2.66 \mathrm{E}+00$ & & & $8.80 \mathrm{E}+00$ & & & $1.32 \mathrm{E}+00$ & & $2.69 \mathrm{E}-02$ & $2.01 E+00$ & $1.34 \mathrm{E}-02$ & $4.51 \mathrm{E}-02$ & $9.23 \mathrm{E}+00$ & $4.88 \mathrm{E}-03$ \\
\hline
\end{tabular}

(1) A blank indicates no activity detected

\begin{tabular}{|lccrr|}
\hline \multicolumn{4}{|l|}{ Ratio Using All Individual Activities } & \\
& Pu-240 & Cs-137 & & Ratio \\
\cline { 2 - 3 } Average & 0.17 & 4.92 & & 0.035 \\
Std. Dev. & 0.48 & 6.70 & $+1-$ & 0.036 \\
95\% Conf. & 0.17 & 1.50 & & \\
\hline
\end{tabular}




\section{ATTACHMENT B}

\section{Pu-241 Ratio Calcs for LLW}

\begin{tabular}{|c|c|c|c|c|c|c|c|c|c|c|c|c|c|c|c|}
\hline \multirow[b]{2}{*}{ Farm/Area } & \multicolumn{3}{|c|}{ Sample Set \#3 } & \multicolumn{3}{|c|}{ Sample Set \#4 } & \multicolumn{3}{|c|}{ Sample Set $\# 5$} & \multicolumn{3}{|c|}{ Sample Set \#6 } & \multicolumn{3}{|c|}{ Sample Set \#7 } \\
\hline & Pu-24I & Cs-137 & Ratio & Pu-24l & Cs- 137 & Ratio & Pu-241 & Cs- 137 & Ratio & $\mathrm{Pu}-241$ & Cs-137 & Ratio & Pu-241 & Cs-137 & Ratio \\
\hline 241-AN & & $5.88 \mathrm{E}-01$ & & & $6.51 \mathrm{E}+00$ & & & $4.59 \mathrm{E}+00$ & & $8.19 \mathrm{E}-01$ & $4.09 \mathrm{E}+00$ & $2.00 \mathrm{E}-01$ & $6.06 \mathrm{E}-01$ & $5.07 \mathrm{E}+00$ & $1.20 \mathrm{E}-01$ \\
\hline 241-AP & & $1.73 \mathrm{E}+00$ & & & $6.49 \mathrm{E}+00$ & & & $3.36 \mathrm{E}+00$ & & $2.60 \mathrm{E}-01$ & $1.75 \mathrm{E}+00$ & $1.49 \mathrm{E}-01$ & $4.10 \mathrm{E}+00$ & $7.47 \mathrm{E}+00$ & $5.48 \mathrm{E}-01$ \\
\hline 241-AW & & $2.07 E+00$ & & & $4.83 \mathrm{E}+00$ & & & $8.74 \mathrm{E}-01$ & & & & & $4.92 \mathrm{E}-01$ & $3.28 \mathrm{E}+00$ & $1.50 \mathrm{E}-01$ \\
\hline 241-AY & & 3.30E-01 & & & $7.36 \mathrm{E}+00$ & & & $2.70 \mathrm{E}+00$ & & $9.59 \mathrm{E}-01$ & $1.00 \mathrm{E}+00$ & $9.59 \mathrm{E}-01$ & $5.16 \mathrm{E}-01$ & $1.60 \mathrm{E}+00$ & 3.23E-01 \\
\hline $244-A$ & & $6.09 \mathrm{E}-01$ & & & $3.25 \mathrm{E}+00$ & & & $<.18$ & & & & & $2.04 E+00$ & $2.64 \mathrm{E}+00$ & 7.71E-01 \\
\hline $242-\mathrm{A}$ & & $4.20 \mathrm{E}+00$ & & & $9.65 \mathrm{E}+00$ & & & $1.54 \mathrm{E}+00$ & & $6.88 \mathrm{E}-02$ & $9.99 \mathrm{E}-01$ & $6.89 \mathrm{E}-02$ & $1.11 E+00$ & $4.39 \mathrm{E}+00$ & $2.52 \mathrm{E}-01$ \\
\hline 204-AR & $8.64 \mathrm{E}-01$ & $7.77 \mathrm{E}+00$ & $1.11 \mathrm{E}-01$ & & $7.19 \mathrm{E}+00$ & & & $2.49 \mathrm{E}+00$ & & $8.07 \mathrm{E}-01$ & $1.52 \mathrm{E}+01$ & $5.31 \mathrm{E}-02$ & $8.11 E+00$ & $2.14 \mathrm{E}+00$ & $3.79 \mathrm{E}+00$ \\
\hline 241-B & & $1.12 \mathrm{E}+01$ & & & $9.45 E+00$ & & & $1.14 E+00$ & & 4.34E-01 & $4.07 E+00$ & $1.07 \mathrm{E}-01$ & 2.57E-01 & $2.30 \mathrm{E}+00$ & $1.12 \mathrm{E}-01$ \\
\hline 241-BX/BY & & $1.37 \mathrm{E}+01$ & & & $8.61 \mathrm{E}+00$ & & & $6.90 \mathrm{E}+00$ & & $5.53 \mathrm{E}-02$ & $4.63 \mathrm{E}+00$ & 1.19E-02 & $1.41 \mathrm{E}-01$ & $2.08 \mathrm{E}+00$ & $6.78 \mathrm{E}-02$ \\
\hline 241-C & & $5.45 \mathrm{E}+01$ & & & $4.06 \mathrm{E}+00$ & & & $1.88 \mathrm{E}+00$ & & $1.32 E+00$ & $7.65 \mathrm{E}+00$ & $1.73 \mathrm{E}-01$ & $9.38 \mathrm{E}-02$ & $1.02 \mathrm{E}+00$ & $9.20 \mathrm{E}-02$ \\
\hline $209-E$ & & $2.84 \mathrm{E}+00$ & & & $1.76 \mathrm{E}+01$ & & & $5.06 \mathrm{E}+00$ & & & $1.30 \mathrm{E}+00$ & & $1.09 \mathrm{E}+00$ & $8.54 \mathrm{E}+00$ & $1.28 \mathrm{E}-01$ \\
\hline 241-S/SX & & $9.11 \mathrm{E}-01$ & & & $2.17 \mathrm{E}+00$ & & $1.90 \mathrm{E}+00$ & $1.48 \mathrm{E}+00$ & $1.28 \mathrm{E}+00$ & $1.66 \mathrm{E}-01$ & $1.66 \mathrm{E}+00$ & 9.97E-02 & $1.52 \mathrm{E}+00$ & $3.45 \mathrm{E}+00$ & 4.39E-01 \\
\hline $241-S$ & & $3.25 \mathrm{E}+00$ & & & $2.51 \mathrm{E}+00$ & & & $1.32 \mathrm{E}+00$ & & $3.17 \mathrm{E}-01$ & $2.91 \mathrm{E}+00$ & $1.09 \mathrm{E}-01$ & $2.29 \mathrm{E}-01$ & $4.81 \mathrm{E}+00$ & 4.77E-02 \\
\hline 241-T & & $1.41 \mathrm{E}+00$ & & $2.95 \mathrm{E}+01$ & $7.77 \mathrm{E}+00$ & $3.80 \mathrm{E}+00$ & & $2.34 \mathrm{E}+00$ & & 7.33E-02 & $6.03 \mathrm{E}-01$ & $1.22 \mathrm{E}-01$ & $3.18 \mathrm{E}-01$ & $6.28 \mathrm{E}+00$ & $5.06 \mathrm{E}-02$ \\
\hline 241-TX/TY & & $6.19 E+00$ & & & $5.47 \mathrm{E}-01$ & & & $1.48 \mathrm{E}+00$ & & & $4.58 \mathrm{E}-01$ & & $3.61 \mathrm{E}-02$ & $6.88 \mathrm{E}+00$ & $5.25 \mathrm{E}-03$ \\
\hline $241-U$ & & $2.66 \mathrm{E}+00$ & & & $8.80 E+00$ & & & $1.32 \mathrm{E}+00$ & & $2.96 \mathrm{E}-0 \mathrm{l}$ & $2.01 \mathrm{E}+00$ & 1.47E-01 & $4.96 \mathrm{E}-01$ & $9.23 \mathrm{E}+00$ & $5.37 \mathrm{E}-02$ \\
\hline
\end{tabular}

(i) A blank indicates no activity detected

\begin{tabular}{|c|c|c|c|c|}
\hline \multicolumn{5}{|c|}{ Ratio Using All Individual Activities } \\
\hline & $\mathrm{Pu}-241$ & Cs- 137 & & Ratio \\
\hline Average & 1.90 & 4.92 & & 0.387 \\
\hline Std. Dev. & 5.27 & 6.70 & $+/-$ & 0.395 \\
\hline $95 \%$ Conf. & 1.86 & 1.50 & & \\
\hline
\end{tabular}




\section{Pu-242 Ratio Calcs for LLW}

\begin{tabular}{|c|c|c|c|c|c|c|c|c|c|c|c|c|c|c|c|}
\hline \multirow[b]{2}{*}{ Farm/Area } & \multicolumn{3}{|c|}{ Sample Set \#3 } & \multicolumn{3}{|c|}{ Sample Set \#4 } & \multicolumn{3}{|c|}{ Sample Set \#5 } & \multicolumn{3}{|c|}{ Sample Set \#6 } & \multicolumn{3}{|c|}{ Sample Set $\# 7$} \\
\hline & $\mathrm{Pu}-242$ & Cs- 137 & Ratio & Pu-242 & Cs-137 & Ratio & $\mathrm{Pu}-242$ & Cs-137 & Ratio & $\mathrm{Pu}-242$ & Cs-137 & Ratio & $\mathrm{Pu}-242$ & Cs-137 & Ratio \\
\hline 241-AN & & $5.88 \mathrm{E}-01$ & & & $6.51 \mathrm{E}+00$ & & & $4.59 \mathrm{E}+00$ & & 3.31E-05 & $4.09 E+00$ & $8.10 \mathrm{E}-06$ & $2.45 \mathrm{E}-05$ & $5.07 \mathrm{E}+00$ & $4.83 \mathrm{E}-06$ \\
\hline 241-AP & & $1.73 E+00$ & & & $6.49 \mathrm{E}+00$ & & & $3.36 \mathrm{E}+00$ & & $1.05 \mathrm{E}-05$ & $1.75 \mathrm{E}+00$ & 6.01E-06 & $1.66 \mathrm{E}-04$ & $7.47 \mathrm{E}+00$ & $2.22 \mathrm{E}-05$ \\
\hline 241-AW & & $2.07 \mathrm{E}+00$ & & & $4.83 E+00$ & & & $8.74 \mathrm{E}-01$ & & & & & $1.99 \mathrm{E}-05$ & $3.28 \mathrm{E}+00$ & $6.06 \mathrm{E}-06$ \\
\hline 241-AY & & $3.30 \mathrm{E}-01$ & & & $7.36 \mathrm{E}+00$ & & & $2.70 \mathrm{E}+00$ & & $3.88 \mathrm{E}-05$ & $1.00 \mathrm{E}+00$ & $3.88 \mathrm{E}-05$ & $2.09 \mathrm{E}-05$ & $1.60 \mathrm{E}+00$ & 1.30E-05 \\
\hline 244-A & & $6.09 \mathrm{E}-01$ & & & $3.25 \mathrm{E}+00$ & & & $<.18$ & & & & & $8.23 E-05$ & $2.64 \mathrm{E}+00$ & $3.12 \mathrm{E}-05$ \\
\hline $242-A$ & & $4.20 \mathrm{E}+00$ & & & $9.65 \mathrm{E}+00$ & & & $1.54 \mathrm{E}+00$ & & $2.78 \mathrm{E}-06$ & $9.99 \mathrm{E}-01$ & $2.78 \mathrm{E}-06$ & 4.47E-05 & $4.39 \mathrm{E}+00$ & $1.02 \mathrm{E}-05$ \\
\hline 204-AR & $3.49 \mathrm{E}-05$ & $7.77 \mathrm{E}+00$ & $4.50 \mathrm{E}-06$ & & $7.19 \mathrm{E}+00$ & & & $2.49 \mathrm{E}+00$ & & $3.26 \mathrm{E}-05$ & $1.52 \mathrm{E}+01$ & $2.15 \mathrm{E}-06$ & $3.28 \mathrm{E}-04$ & $2.14 \mathrm{E}+00$ & $1.53 \mathrm{E}-04$ \\
\hline 241-B & & $1.12 \mathrm{E}+01$ & & & $9.45 \mathrm{E}+00$ & & & $1.14 \mathrm{E}+00$ & & $1.76 \mathrm{E}-05$ & $4.07 \mathrm{E}+00$ & 4.31E-06 & $1.04 \mathrm{E}-05$ & $2.30 \mathrm{E}+00$ & $4.52 \mathrm{E}-06$ \\
\hline 241-BX/BY & & $1.37 \mathrm{E}+01$ & & & $8.61 E+00$ & & & $6.90 \mathrm{E}+00$ & & $2.24 \mathrm{E}-06$ & $4.63 E+00$ & $4.83 \mathrm{E}-07$ & $5.70 \mathrm{E}-06$ & $2.08 \mathrm{E}+00$ & $2.74 \mathrm{E}-06$ \\
\hline $241-C$ & & $5.45 \mathrm{E}+01$ & & & $4.06 \mathrm{E}+00$ & & & $1.88 \mathrm{E}+00$ & & 5.35E-05 & $7.65 \mathrm{E}+00$ & $6.99 \mathrm{E}-06$ & $3.79 \mathrm{E}-06$ & $1.02 \mathrm{E}+00$ & $3.72 \mathrm{E}-06$ \\
\hline 209-E & & $2.84 \mathrm{E}+00$ & & & $1.76 \mathrm{E}+01$ & & & $5.06 \mathrm{E}+00$ & & & $1.30 \mathrm{E}+00$ & & $4.42 \mathrm{E}-05$ & $8.54 \mathrm{E}+00$ & $5.18 \mathrm{E}-06$ \\
\hline 241-S/SX & & $9.11 \mathrm{E}-01$ & & & $2.17 \mathrm{E}+00$ & & $7.68 \mathrm{E}-05$ & $1.48 \mathrm{E}+00$ & $5.19 \mathrm{E}-05$ & $6.69 \mathrm{E}-06$ & $1.66 \mathrm{E}+00$ & $4.03 E-06$ & $6.13 \mathrm{E}-05$ & $3.45 E+00$ & 1.78E-05 \\
\hline 241-SY & & $3.25 \mathrm{E}+00$ & & & $2.51 \mathrm{E}+00$ & & & $1.32 \mathrm{E}+00$ & & $1.28 \mathrm{E}-05$ & $2.91 \mathrm{E}+00$ & $4.40 \mathrm{E}-06$ & $9.27 \mathrm{E}-06$ & $4.81 \mathrm{E}+00$ & $1.93 \mathrm{E}-06$ \\
\hline 241-T & & $1.41 \mathrm{E}+00$ & & $1.19 \mathrm{E}-03$ & $7.77 \mathrm{E}+00$ & $1.54 \mathrm{E}-04$ & & $2.34 \mathrm{E}+00$ & & $2.96 \mathrm{E}-06$ & $6.03 \mathrm{E}-01$ & 4.92E-06 & $1.28 \mathrm{E}-05$ & $6.28 \mathrm{E}+00$ & $2.04 \mathrm{E}=06$ \\
\hline $241 \cdot \mathrm{TX} / \mathrm{TY}$ & & $6.19 \mathrm{E}+00$ & & & $5.47 \mathrm{E}-01$ & & & $1.48 \mathrm{E}+00$ & & & $4.58 \mathrm{E}-01$ & & $1.46 \mathrm{E}-06$ & $6.88 E+00$ & $2.12 \mathrm{E}-07$ \\
\hline $241-\mathrm{U}$ & & $2.66 \mathrm{E}+00$ & & & $8.80 \mathrm{E}+00$ & & & $1.32 \mathrm{E}+00$ & & $1.20 \mathrm{E}-05$ & $2.01 E+00$ & $5.96 \mathrm{E}-06$ & $2.00 \mathrm{E}-05$ & $9.23 \mathrm{E}+00$ & 2.17E-06 \\
\hline
\end{tabular}

(1) A blank indicates no activity detected

\begin{tabular}{|c|c|c|c|c|}
\hline \multicolumn{5}{|c|}{ Ratio Using All Individual Activities } \\
\hline & $\mathrm{Pu}-242$ & Cs-137 & & Ratio \\
\hline Average & $7.70 \mathrm{E}-05$ & 4.92 & & $1.56 \mathrm{E}-05$ \\
\hline Std. Dev. & $2.13 \mathrm{E}-04$ & 6.70 & +1 & $1.60 \mathrm{E}-05$ \\
\hline $95 \%$ Conf. & $7.50 \mathrm{E}-05$ & 1.50 & & \\
\hline
\end{tabular}


ATTACHMENT B

WHC-SD-WM-ER-435, Rev. 1

Page B-29 of 29

Am-241 Ratios

$\mathrm{pCi} / \mathrm{g}$

Sample Set \#6

Sample Set \#7

\begin{tabular}{l} 
Farm/Area \\
\hline \hline \\
$241-A N$ \\
$241-A P$ \\
$241-A W$ \\
$241-A Y$ \\
$244-A$ \\
$242-A$ \\
$204-A R$ \\
$241-B$ \\
$241-B X / B Y$ \\
$241-C$ \\
$209-E$ \\
$241-S / S X$ \\
$241-S Y$ \\
$241-T$ \\
$241-T X / T Y$ \\
$241-U$
\end{tabular}

Am-241

Cs-137

Am-241 Cs-137

$1.87 \mathrm{E}-01 \quad 4.09 \mathrm{E}+00$

$1.16 \mathrm{E}-01 \quad 5.07 \mathrm{E}+00$

$7.14 \mathrm{E}-02 \quad 1.75 \mathrm{E}+00$

$1.33 \mathrm{E}+00 \quad 7.47 \mathrm{E}+00$

$9.30 \mathrm{E}-02 \quad 3.28 \mathrm{E}+00$

$1.09 \mathrm{E}-01 \quad 1.00 \mathrm{E}+00$

$1.08 \mathrm{E}-01 \quad 1.60 \mathrm{E}+00$

2.05E-02 9.99E-01

$3.91 \mathrm{E}-01 \quad 2.64 \mathrm{E}+00$

$2.03 \mathrm{E}-01 \quad 1.52 \mathrm{E}+01$

$2.23 \mathrm{E}-01 \quad 4.39 \mathrm{E}+00$

$1.07 \mathrm{E}-01 \quad 4.07 \mathrm{E}+00$

$5.60 \mathrm{E}-01 \quad 2.14 \mathrm{E}+00$

$9.72 \mathrm{E}-02 \quad 4.63 \mathrm{E}+00$

$7.96 \mathrm{E}-02 \quad 2.30 \mathrm{E}+00$

$3.87 \mathrm{E}-01 \quad 7.65 \mathrm{E}+00$

$3.57 \mathrm{E}-02 \quad 2.08 \mathrm{E}+00$

$<1.50 \mathrm{E}-02 \quad 1.30 \mathrm{E}+00$

$4.08 \mathrm{E}-02 \quad 1.02 \mathrm{E}+00$

$<2.20 \mathrm{E}-02 \quad 1.66 \mathrm{E}+00$

$2.71 \mathrm{E}-01 \quad 8.54 \mathrm{E}+00$

4.63E-02 2.91E+00

$2.02 \mathrm{E}-01 \quad 3.45 \mathrm{E}+00$

$<1.10 \mathrm{E}-02 \quad 6.03 \mathrm{E}-01$

$3.35 \mathrm{E}-02 \quad 4.81 \mathrm{E}+00$

$3.79 \mathrm{E}-02 \quad 6.28 \mathrm{E}+00$

$2.34 \mathrm{E}-02 \quad 4.58 \mathrm{E}-0 \mathrm{I}$

$1.19 \mathrm{E}-02 \quad 6.88 \mathrm{E}+00$

8.38E-02 2.01E +00

$6.41 \mathrm{E}-02 \quad 9.23 \mathrm{E}+00$

\begin{tabular}{|c|c|c|c|c|}
\hline \multicolumn{5}{|c|}{ Ratio Using All Individual Activities } \\
\hline & $\mathrm{Am}=241$ & Cs-137 & & Ratio \\
\hline Average & 0.18 & 3.98 & & 0.046 \\
\hline Std. Dev. & 0.26 & 3.20 & $+1-$ & 0.028 \\
\hline $95 \%$ Conf. & 0.10 & 1.14 & & \\
\hline
\end{tabular}


WHC-SD-WM-ER-435, Rev. 1

Page $\mathrm{C}-\mathrm{i}$

\section{ATTACHMENT C}


LLW TO ATG

SHIPMENT DATE: 1-19-95

\begin{tabular}{|c|c|c|c|c|c|c|c|}
\hline PIN \# & $6 \mathrm{pt}$ & Origin & Weight & Volume & \multicolumn{2}{|c|}{ ASSAY } & \multirow[t]{3}{*}{ CONTENTS } \\
\hline & $(\mathrm{mr} / \mathrm{hr})$ & & $(\mathrm{Kg})$ & $\left(m^{3}\right)$ & Cs-137 & Co-60 & \\
\hline & & \multicolumn{5}{|c|}{$(p C i / g)$} & \\
\hline 94-108-01 & 0.15 & A & 463.0 & 4.0 & 26 & $<.57$ & Plastic, Metal, Paper, Fiber Glass \\
\hline $93-316-01$ & $<0.5$ & AY & 415.0 & 4.0 & 270.0 & 0.91 & Plastic, Cloth, Rubber, Paper \\
\hline $94-089-02$ & $<0.5$ & AY & 279.0 & 4.0 & 22.0 & 2.10 & Plastic, Cloth, Rubber, Paper \\
\hline $94-108-02$ & $<0.5$ & BXVBY & 469.5 & 4.0 & 130.0 & 0.63 & Plastic, Cloth, Rubber, Paper \\
\hline $94-136-03$ & $<0.5$ & BX/BY & 460.4 & 4.0 & 4600.0 & 0.32 & Plastic, Cloth, Rubber \\
\hline $94-129-01$ & $<0.5$ & C & 406.0 & 4.0 & 87.0 & 0.35 & Plastic, Cloth, Rubber, Paper \\
\hline $94-137-02$ & $<0.5$ & C & 460.4 & 4.0 & 610.0 & 0.38 & Plastic, Cloth, Rubber, Paper \\
\hline $94-109-01$ & $<0.5$ & $\mathrm{~S}$ & 369.7 & 4.0 & 340.0 & 2.50 & Plastic, Cloth, Rubber, Paper \\
\hline $94-130-05$ & $<0.5$ & $T$ & 587.4 & 4.0 & 0.6 & $<0.28$ & Wood \\
\hline
\end{tabular}


LLW TO ATG

SHIPMENT DATE: $1-26-95$

\begin{tabular}{|c|c|c|c|c|c|c|}
\hline PIN\# & $6 \mathrm{pt}$ & Origin & Weight & Volume & ASSAY & CONTENTS \\
\hline & $(\mathrm{mr} / \mathrm{hr})$ & & $(\mathrm{Kg})$ & $\left(m^{3}\right)$ & Cs-137 Co-60 & \\
\hline
\end{tabular}

$\begin{array}{llcccccl}93-215-03 & <0.5 & \text { AW } & 292.6 & 4.0 & 240.0 & 3.25 & \text { Plastic, Cloth, Rubber, Paper } \\ 94-159-04 & <0.5 & \text { AY } & 351.5 & 4.0 & 79.0 & <1.2 & \text { Plastic, Cloth, Rubber, Paper, Absorbent } \\ 94-151-01 & <0.5 & \text { BX/BY } & 192.8 & 4.0 & 630.0 & 6.2 & \text { Plastic, Cloth, Rubber, Paper } \\ 93-278-01 & 5.00 & \text { C } & 412.8 & 4.0 & 17,000 & <.56 & \text { Plastic, Wood, Paper, Vegetation } \\ 94-147-01 & <0.5 & \text { C } & 238.1 & 4.0 & 210.0 & 2.7 & \text { Plastic, Cloth, Rubber, Paper, Wood } \\ 94-173-02 & <0.5 & \text { C } & 260.8 & 4.0 & 1200.0 & 2.35 & \text { Plastic, Cloth, Rubber, Paper } \\ 94-139-03 & <0.5 & \text { S } & 374.2 & 4.0 & 670.2 & 7.5 & \text { Plastic, Cloth, Rubber, Paper, Vegetation } \\ 94-082-02 & <0.5 & \text { SY } & 238.1 & 4.0 & 810.0 & 2.0 & \text { Plastic, Cloth, Rubber, Paper, Asbestos } \\ 93-364-01 & <0.5 & \text { TXTY } & 374.2 & 4.0 & 86.0 & 1.32 & \text { Plastic, Cloth, Rubber, Paper, Absorbent } \\ 93-327-02 & <0.5 & \text { U } & 328.9 & 4.0 & 320.0 & 1.1 & \text { Plastic, Cloth, Rubber, Paper, Wood, Soil }\end{array}$


LLW TO ATG

SHIPMENT DATE: 2-2-95

\begin{tabular}{|c|c|c|c|c|c|c|}
\hline PIN \# & $6 \mathrm{pt}$ & Origin & Weight & Volume & ASSAY & CONTENTS \\
\hline & r/hr) & & $(\mathrm{Kg})$ & $\left(\mathrm{m}^{3}\right)$ & Cs-137 Co-60 & \\
\hline
\end{tabular}

$\begin{array}{lllllccl}94-122-01 & <0.5 & \text { A } & 460.4 & 4.0 & 73.0 & 1.0 & \text { Plastic, Cloth, Rubber, Paper, Metal, Vegetation } \\ 94-082-03 & <0.5 & \text { AR } & 387.8 & 4.0 & 41 & <0.6 & \text { Plastic, Cloth, Rubber, Paper, Metal } \\ & & \text { AR } & & & \text { Eu-154 } & 8.2 & \\ 93-105-24 & <0.5 & \text { B } & 56.7 & 4.0 & 390.0 & 7.8 & \text { Plastic, Cloth, Rubber, Paper, Metal } \\ & & \text { B } & & & \text { Eu-154 } & 17.0 & \\ 94-178-01 & <0.5 & \text { BX/BY } & 283.5 & 4.0 & 250.0 & 4.2 & \text { Plastic, Cloth, Rubber, Paper, Absorbent } \\ 94-193-02 & <0.5 & \text { C } & 308.0 & 4.0 & 640.0 & 1.20 & \text { Plastic, Cloth, Rubber, Paper, Metal } \\ 94-208-03 & <0.5 & \text { C } & 347.0 & 4.0 & 970.0 & <1.0 & \text { Plastic, Cloth, Rubber, Paper, Metal } \\ & & \text { C } & & & \text { Eu-154 } & 1.3 & \\ 94-168-01 & <0.5 & \text { SX } & 460.4 & 4.0 & 98.0 & 2.3 & \text { Plastic, Cloth, Rubber, Paper, Metal } \\ & & \text { SX } & & & \text { Eu-154 } & 1.5 & \\ 94-171-01 & <0.5 & \text { SY } & 319.8 & 4.0 & 1200.0 & 0.6 & \text { Plastic, Cloth, Rubber, Paper, Metal } \\ & & \text { SY } & & & \text { Eu-154 } & 1.7 & \\ 94-125-02 & <0.5 & \text { T } & 433.2 & 4.0 & 110.0 & 3.20 & \text { Plastic, Cloth, Rubber, Paper, Metal, Wood } \\ & & \text { T } & & & \text { Eu-154 } & 6.2 & \\ 94-206-02 & <0.5 & \text { U } & 269.9 & 4.0 & 200.0 & 0.7 & \text { Plastic, Cloth, Rubber, Paper, Metal }\end{array}$


LLW TO ATG

SHIPMENT DATE: 2-7-95

\begin{tabular}{ccccccc}
\hline PIN\# & $6 \mathrm{pt}$ & Origin & Weight & Volume & ASSAY & CONTENTS \\
\hline & $(\mathrm{mr} / \mathrm{hr})$ & & $(\mathrm{Kg})$ & $\left(\mathrm{m}^{3}\right)$ & $\mathrm{Cs}-137 \quad \mathrm{Co}-60$ \\
& & & $(\mathrm{pCi} / \mathrm{g})$
\end{tabular}

$\begin{array}{llclllll}94-159-03 & <0.5 & \text { A } & 351.5 & 4.0 & 49.0 & 0.88 & \text { Plastic, Cloth, Rubber, Paper, Metal, Vegetation } \\ 94-143-02 & <0.5 & \text { AN } & 337.9 & 4.0 & 23.0 & 1.45 & \text { Plastic, Cloth, Rubber, Paper, Wood, Metal } \\ 94-208-02 & <0.5 & \text { AW } & 365.1 & 4.0 & 870.0 & 2.4 & \text { Plastic, Cloth, Rubber, Paper, Wood, Metal } \\ 94-206-01 & <0.5 & \text { BX/BY } & 446.8 & 4.0 & 1000.0 & 1.0 & \text { Plastic, Cloth, Rubber, Paper, Metal } \\ 94-242-01 & <0.5 & \text { C } & 342.5 & 4.0 & 410.0 & 1.8 & \text { Plastic, Cloth, Rubber, Paper, Metal } \\ & & \text { C } & & & \text { Eu-154 } & 3.5 & \\ 94-250-01 & <0.5 & \text { S } & 587.4 & 4.0 & 46.0 & 0.8 & \text { Plastic, Cloth, Rubber, Paper, Wood, Metal } \\ 94-227-01 & <0.5 & \text { S/SX } & 229.1 & 4.0 & 180.0 & 2.9 & \text { Plastic, Cloth, Rubber, Paper, Metal } \\ 94-249-02 & <0.5 & \text { SISX } & 337.9 & 4.0 & 1700.0 & 3.10 & \text { Plastic, Cloth, Rubber, Paper, Metal } \\ 94-249-01 & <0.5 & \text { SY } & 265.4 & 4.0 & 5600.0 & 2.3 & \text { Plastic, Cloth, Rubber, Paper, Metal } \\ 94-227-02 & <0.5 & \text { U } & 315.3 & 4.0 & 810.0 & 1.0 & \text { Plastic, Cloth, Rubber, Paper, Wood, Metal } \\ & & \text { U } & & & \text { Eu-154 } & 10.5 & \end{array}$


LLW TO ATG

SHIPMENT DATE: 2-9-95

\begin{tabular}{|c|c|c|c|c|c|c|}
\hline PIN \# & $6 \mathrm{pt}$ & Origin & Weight & Volume & ASSAY & CONTENTS \\
\hline & $(\mathrm{mr} / \mathrm{hr})$ & & $(\mathrm{Kg})$ & $\left(m^{3}\right)$ & Cs-137 Co-60 & \\
\hline
\end{tabular}

$\begin{array}{cccccccc}94-208-04 & <0.5 & \text { AY } & 224.5 & 4.0 & 260.0 & 2.80 & \text { Plastic, Cloth, Rubber, Paper, Wood, Metal } \\ 94-271-03 & <0.5 & \text { AY } & 374.2 & 4.0 & 3000.0 & 1.8 & \text { Plastic, Cloth, Rubber, Paper, Wood, Metal } \\ 94-270-09 & <0.5 & \text { BX/BY } & 360.6 & 4.0 & 910.0 & 2.0 & \text { Plastic, Cloth, Rubber, Paper, Metal } \\ & & \text { BX/BY } & & & \text { Eu-154 } & 23.75 & \\ 94-270-01 & <0.5 & \text { C } & 337.9 & 4.0 & 6.8 & 0.4 & \text { Plastic, Cloth, Rubber, Paper, Wood, Metal } \\ 94-279-01 & <0.5 & \text { C } & 383.3 & 4.0 & 78.0 & 0.8 & \text { Plastic, Cloth, Rubber, Paper, Wood, Metal } \\ & & \text { C } & & & \text { Eu-154 } & 3.5 & \\ 94-292-02 & <0.5 & \text { C } & 601.0 & 4.0 & 180.0 & 1.40 & \text { Plastic, Cloth, Rubber, Paper, Metal } \\ 94-298-01 & <0.5 & \text { C } & 328.9 & 4.0 & 140.0 & 3.0 & \text { Plastic, Cloth, Rubber, Paper, Metal } \\ 94-277-01 & <0.5 & \text { S/SX } & 374.2 & 4.0 & 530.0 & 3.2 & \text { Plastic, Cloth, Rubber, Paper, Wood, Metal } \\ 94-269-02 & <0.5 & \text { SY } & 238.1 & 4.0 & 88.0 & 1.5 & \text { Plastic, Cloth, Rubber, Paper, Metal } \\ & & \text { SY } & & & \text { Eu-154 } & 3.8 & \\ 94-206-03 & <0.5 & \text { TX/TY } & 260.8 & 4.0 & 460.0 & 2.90 & \text { Plastic, Cloth, Rubber, Paper, Metal, Vegetation }\end{array}$




\section{LLW TO ATG}

SHIPMENT DATE: 2-14-95

\begin{tabular}{|c|c|c|c|c|c|c|c|}
\hline PIN \# & $6 \mathrm{pt}$ & Origin & Weight & Volume & \multicolumn{2}{|c|}{ ASSAY } & \multirow[t]{2}{*}{ CONTENTS } \\
\hline & (mr/hr) & & $(\mathrm{Kg})$ & $\left(m^{3}\right)$ & Cs-137 & Co-60 & \\
\hline \multicolumn{8}{|c|}{$(\mathrm{pCi} / \mathrm{g})$} \\
\hline $94-180-03$ & $<0.5$ & A & 510.3 & 4.0 & 870.0 & 0.41 & Plastic, Cloth, Rubber, Paper, Wood, Metal \\
\hline $94-082-04$ & $<0.5$ & AP & 605.6 & 4.0 & 1200.0 & 340.00 & Plastic, Cloth, Rubber, Paper, Metal, Concrete \\
\hline \multicolumn{8}{|c|}{ Cs-134 2.9} \\
\hline $94-252-01$ & $<0.5$ & BX/BY & 260.8 & 4.0 & 860.0 & 1.7 & Plastic, Cloth, Rubber, Paper, Metal \\
\hline \multicolumn{8}{|c|}{ Eu-154 8.5} \\
\hline $94-270-09$ & $<0.5$ & $\mathrm{BX} / \mathrm{BY}$ & 360.6 & 4.0 & 910.0 & 2.0 & Plastic, Cloth, Rubber, Paper, Metal \\
\hline $94-304-03$ & $<0.5$ & C & 601.0 & 4.0 & 170.0 & 3.1 & Plastic, Cloth, Rubber, Paper, Metal, Leather \\
\hline $94-306-01$ & $<0.5$ & $\mathrm{C}$ & 528.4 & 4.0 & 480.0 & 1.1 & Plastic, Cloth, Rubber, Paper, Wood, Metal \\
\hline $94-315-01$ & $<0.5$ & C & 360.6 & 4.0 & 530.0 & 1.20 & Plastic, Cloth, Rubber, Paper, Metal \\
\hline \multicolumn{8}{|c|}{ Eu-154 6.25} \\
\hline $94-327-05$ & $<0.5$ & C & 283.5 & 4.0 & 850.0 & 0.69 & Plastic, Cloth, Rubber, Paper, Metal \\
\hline \multicolumn{8}{|c|}{ Eu-154 2.3} \\
\hline $94-304-01$ & $<0.5$ & S/SX & 374.2 & 4.0 & 1400.0 & 3.5 & Plastic, Cloth, Rubber, Paper, Metal \\
\hline \multicolumn{8}{|c|}{ Eu-154 4.5} \\
\hline $94-321-01$ & $<0.5$ & S/SX & 297.1 & 4.0 & 270.0 & 4.3 & Plastic, Cloth, Rubber, Paper, Metal \\
\hline $94-294-01$ & $<0.5$ & SY & 392.4 & 4.0 & 92.0 & 0.6 & Plastic, Cloth, Rubber, Paper, Metal, Insulation \\
\hline
\end{tabular}




\section{LLW TO ATG}

SHIPMENT DATE: 2-22-95

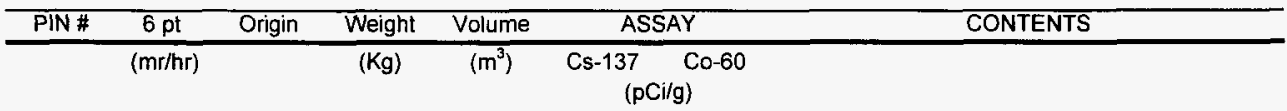

\begin{tabular}{|c|c|c|c|c|c|c|c|}
\hline $94-213-02$ & $<0.5$ & 242-A EVAP & 437.7 & 4.0 & 240.0 & 0.99 & Plastic, Cloth, Rubber, Paper, Metal, Insulation \\
\hline $94-263-02$ & $<0.5$ & AN & 238.1 & 4.0 & 1000.0 & 3.2 & Plastic, Cloth, Rubber, Paper, Metal \\
\hline $94-264-02$ & $<0.5$ & AW & 179.2 & 4.0 & 390.0 & 3.1 & Plastic, Cloth, Rubber, Paper, Metal \\
\hline $94-340-03$ & $<0.5$ & AY & 188.2 & 4.0 & 140.0 & $<1.0$ & Plastic, Cloth, Rubber, Paper, Wood, Metal \\
\hline $94-327-06$ & $<0.5$ & $\mathrm{BX} / \mathrm{BY}$ & 224.5 & 4.0 & 1600.0 & 1.8 & Plastic, Cloth, Rubber, Paper, Metal \\
\hline $94-339-02$ & $<0.5$ & $\mathrm{~S} / \mathrm{SX}$ & 265.4 & 4.0 & 42.0 & 1.3 & Plastic, Cloth, Rubber, Paper, Metal \\
\hline $94-339-01$ & $<0.5$ & SY & 220.0 & 4.0 & 32.0 & $<1.0$ & Plastic, Cloth, Rubber, Paper, Metal, Insulation \\
\hline \multirow[t]{2}{*}{$94-249-03$} & $<0.5$ & $\mathrm{U}$ & 374.2 & 4.0 & 1500.0 & 0.74 & Plastic, Cloth, Rubber, Paper, Wood, Metal \\
\hline & & $\mathrm{U}$ & & & Eu-154 & 1.8 & \\
\hline
\end{tabular}


LLW TO ATG

SHIPMENT DATE: 3-2-95

\begin{tabular}{|c|c|c|c|c|c|c|c|}
\hline \multirow[t]{2}{*}{ PIN \# } & \multirow{2}{*}{$\frac{6 \mathrm{pt}}{(\mathrm{mr} / \mathrm{hr})}$} & \multirow[t]{2}{*}{ Origin } & \multirow{2}{*}{$\frac{\text { Weight }}{(\mathrm{Kg})}$} & \multirow{2}{*}{$\frac{\text { Voltume }}{\left(\mathrm{m}^{3}\right)}$} & \multicolumn{2}{|c|}{ ASSAY } & \multirow[t]{2}{*}{ CONTENTS } \\
\hline & & & & & Cs-137 & Co-60 & \\
\hline \multicolumn{8}{|c|}{$(p \mathrm{Ci} / \mathrm{g})$} \\
\hline $94-095-01$ & $<0.5$ & 244-AR & 319.8 & 4.0 & 210.0 & 1.20 & Plastic, Cloth, Rubber, Paper, Wood, Metal \\
\hline $94-343-01$ & $<0.5$ & AY & 265.4 & 4.0 & 170.0 & $<1.0$ & Plastic, Cloth, Rubber, Paper, Metal \\
\hline $94-340-06$ & $<0.5$ & BXJBY & 210.9 & 4.0 & 1000.0 & 1.90 & Plastic, Cloth, Rubber, Paper, Wood, Metal \\
\hline $94-356-02$ & $<0.5$ & BX/BY & 283.5 & 4.0 & 1900.0 & 1.0 & Plastic, Cloth, Rubber, Paper, Wood, Metal \\
\hline $94-356-01$ & $<0.5$ & C & 419.6 & 4.0 & 1300.0 & 0.5 & Plastic, Cloth, Rubber, Paper, Metal \\
\hline $94-355-02$ & $<0.5$ & $\mathrm{~S} / \mathrm{SX}$ & 292.6 & 4.0 & 8.8 & 1.1 & Plastic, Cloth, Rubber, Paper, Metal \\
\hline $94-363-01$ & $<0.5$ & $S / S X$ & 310.7 & 4.0 & 180.0 & $<1.0$ & Plastic, Cloth, Rubber, Paper, Metal \\
\hline $94-355-03$ & $<0.5$ & SY & 210.9 & 4.0 & 34.0 & $<1.5$ & Plastic, Cloth, Paper \\
\hline $94-363-01$ & $<0.5$ & SY & 283.5 & 4.0 & 90.0 & 0.8 & Plastic, Cloth, Rubber, Paper, Wood, Metal \\
\hline $94-363-04$ & $<0.5$ & SY & 192.8 & 4.0 & 6.8 & $<1.0$ & Plastic \\
\hline
\end{tabular}


LLW TO ATG

SHIPMENT DATE: 3-16-95

\begin{tabular}{|c|c|c|c|c|c|c|c|}
\hline PIN\# & $6 \mathrm{pt}$ & Origin & Weight & Volume & \multicolumn{2}{|c|}{ ASSAY } & \multirow[t]{2}{*}{ CONTENTS } \\
\hline & $(\mathrm{mr} / \mathrm{hr})$ & & $(\mathrm{Kg})$ & $\left(m^{3}\right)$ & $\mathrm{Cs}-137$ & Co-60 & \\
\hline \multicolumn{8}{|c|}{$(\mathrm{pCi} / \mathrm{g})$} \\
\hline 94-082-01 & $<0.5$ & 209-E & 419.6 & 4.0 & 82.0 & 1.90 & Plastic, Cloth, Rubber, Paper, Metal \\
\hline $94-346-01$ & $<0.5$ & AW & 328.9 & 4.0 & 600.0 & 1.90 & Plastic, Cloth, Rubber, Paper, Metal \\
\hline $95-010-02$ & $<0.5$ & BX/BY & 374.0 & 4.0 & 3200.0 & 2.1 & Plastic, Cloth, Rubber, Paper, Wood, Metal \\
\hline \multicolumn{8}{|c|}{ Eu-154 6.3} \\
\hline $95-012-05$ & $<0.5$ & C & 374.2 & 4.0 & 1200.0 & 1.8 & Plastic, Cloth, Rubber, Paper, Wood, Metal \\
\hline $95-018-05$ & $<0.5$ & C & 306.2 & 4.0 & 3700.0 & 3.1 & Plastic, Cloth, Rubber, Paper, Wood, Metal \\
\hline \multicolumn{8}{|c|}{ Eu-154 2.1} \\
\hline 95-024-02 & $<0.5$ & C & 324.3 & 4.0 & 1500.0 & 5.2 & Plastic, Cloth, Rubber, Paper, Wood, Metal \\
\hline \multicolumn{8}{|c|}{ Eu-154 1.9} \\
\hline 94-264-01 & $<0.5$ & $\mathbf{S}$ & 1327.0 & 4.0 & 4.6 & $<0.19$ & Plastic, Wood, Metal, Concrete \\
\hline 95-005-01 & $<0.5$ & S/SX & 351.5 & 4.0 & 65.0 & $<1.4$ & Plastic, Cloth, Rubber, Paper, Wood, Metal \\
\hline $95-012-04$ & $<0.5$ & sX & 555.7 & 4.0 & 210.0 & $<0.55$ & Plastic, Cloth, Rubber, Paper, Wood, Metal \\
\hline \multicolumn{8}{|c|}{ Eu-154 2.4} \\
\hline $94-355-04$ & $<0.5$ & $\mathbf{U}$ & 328.9 & 4.0 & 1400.0 & 1.0 & Plastic, Cloth, Rubber, Paper, Metal \\
\hline \multicolumn{8}{|c|}{ Eu-154 4.0} \\
\hline
\end{tabular}


LLW TO ATG

SHIPMENT DATE: 3-30-95

\begin{tabular}{|c|c|c|c|c|c|c|c|}
\hline PIN\# & $6 \mathrm{pt}$ & Origin & Weight & Volume & \multicolumn{2}{|c|}{ ASSAY } & CONTENTS \\
\hline & \multirow[t]{2}{*}{$(\mathrm{mr} / \mathrm{hr})$} & & $(\mathrm{Kg})$ & $\left(m^{3}\right)$ & Cs-137 & Co-60 & \\
\hline \multicolumn{7}{|c|}{$(\mathrm{pCi} / \mathrm{g})$} & \\
\hline $95-010-01$ & $<0.5$ & AY & 315.3 & 4.0 & 137.0 & 1.60 & Plastic, Cloth, Rubber, Paper, Wood, Metal \\
\hline \multicolumn{8}{|c|}{ Eu-154 4.3} \\
\hline $94-220-01$ & 1.0 & B & 315.3 & 4.0 & 600.0 & 1.60 & Plastic, Cloth, Rubber, Paper, Wood, Metal \\
\hline $95-025-01$ & 1.0 & BXVYY & 201.9 & 4.0 & 2100.0 & 2.2 & Plastic, Cloth, Rubber, Paper, Wood, Metal \\
\hline $95-038-03$ & $<0.5$ & BX/BY & 315.3 & 4.0 & 495.0 & $<1.0$ & Plastic, Cloth, Rubber, Paper, Wood, Metal \\
\hline \multicolumn{8}{|c|}{ Eu-154 3.5} \\
\hline $95-037-01$ & $<0.5$ & C & 319.8 & 4.0 & 235.0 & 2.8 & Plastic, Cloth, Rubber, Paper, Wood, Metal \\
\hline \multicolumn{8}{|c|}{ Eu-154 2.7} \\
\hline $95-024-01$ & $<0.5$ & $\mathrm{~S} / \mathrm{SX}$ & 420.0 & 4.0 & 70.5 & $<0.5$ & Plastic, Cloth, Rubber, Paper, Wood, Metal, Asbestos \\
\hline $95-038-04$ & $<0.5$ & $\mathrm{~S} / \mathrm{SX}$ & 231.3 & 4.0 & 2000.0 & 3.9 & Plastic, Cloth, Rubber, Paper, Metal \\
\hline \multicolumn{8}{|c|}{ Eu-154 18} \\
\hline $95-012-02$ & 1.8 & SY & 383.3 & 4.0 & 3400.0 & 1.4 & Plastic, Cloth, Rubber, Paper, Wood, Meta), Concrete \\
\hline \multicolumn{8}{|c|}{ Eu-154 2.5} \\
\hline $94-224-01$ & $<0.5$ & $\mathbf{T}$ & 337.9 & 4.0 & 80.0 & 1.2 & Plastic, Cloth, Rubber, Paper, Wood, Metal \\
\hline $95-012-03$ & $<0.5$ & $\mathrm{U}$ & 428.7 & 4.0 & 480.0 & 1.0 & Plastic, Cloth, Rubber, Paper, Wood, Metal \\
\hline \multicolumn{8}{|c|}{ Eu-154 2.1} \\
\hline
\end{tabular}


LLW TO ATG

SHIPMENT DATE: 4-13-95

\begin{tabular}{|c|c|c|c|c|c|c|c|}
\hline PIN\# & $6 \mathrm{pt}$ & Origin & Weight & Volume & \multicolumn{2}{|c|}{$\overline{A S S A ̄ Y}$} & CONTENTS \\
\hline & $(\mathrm{mr} / \mathrm{hr})$ & & $(\mathrm{Kg})$ & $\left(m^{3}\right)$ & $\begin{array}{r}\mathrm{Cs}-137 \\
\quad(\mathrm{pCi} /\end{array}$ & Co-60 & \\
\hline $94-356-03$ & $<0.5$ & $\begin{array}{l}\text { AN } \\
\text { AN }\end{array}$ & 331.1 & 4.0 & $\begin{array}{l}2300.0 \\
\text { Eu-154 }\end{array}$ & $\begin{array}{r}2.6 \\
14.0\end{array}$ & Plastic, Cloth, Rubber, Paper, Metal \\
\hline $94-322-03$ & $<0.5$ & AP & 215.5 & 4.0 & $\begin{array}{l}900.0 \\
\text { Cs-134 }\end{array}$ & $\begin{array}{l}<1.0 \\
4.35\end{array}$ & Plastic, Cloth, Rubber, Paper, Metal, Weeds \\
\hline $95-047-01$ & $<0.5$ & AY & 476.3 & 4.0 & $\begin{array}{l}110.0 \\
\text { Eu-154 }\end{array}$ & $\begin{array}{c}0.9 \\
1.7\end{array}$ & Plastic, Cloth, Rubber, Paper, Wood, Metal, Asbestos \\
\hline $95-055-02$ & $<0.5$ & $\begin{array}{l}\text { AY } \\
\text { AY }\end{array}$ & 344.7 & 4.0 & $\begin{array}{l}49.0 \\
\text { Eu-154 }\end{array}$ & $\begin{aligned} 1.7 \\
77.1\end{aligned}$ & Plastic, Cloth, Rubber, Paper, Wood, Metal \\
\hline $95-044-01$ & $<0.5$ & $\begin{array}{l}\mathrm{C} \\
\mathrm{C}\end{array}$ & 601.0 & 4.0 & $\begin{array}{c}50.0 \\
\text { Eu-154 } 1.2\end{array}$ & $\begin{array}{r}0.83 \\
\mathrm{U}-235\end{array}$ & $\begin{array}{l}\text { Plastic, Cloth, Rubber, Paper, Metal } \\
5.1\end{array}$ \\
\hline 95-059-01 & $<0.5$ & $\begin{array}{l}c \\
c\end{array}$ & 238.1 & 4.0 & $\begin{array}{l}320.0 \\
\text { Th-228 }\end{array}$ & $\begin{array}{l}2.9 \\
3.95\end{array}$ & Plastic, Cloth, Rubber, Paper, Wood, Metal \\
\hline $95-055-03$ & $<0.5$ & $s x$ & 197.3 & 4.0 & 29.0 & $<1.0$ & Plastic, Cloth, Rubber, Paper, Wood, Metal, Concrete \\
\hline $94-294-02$ & $<0.5$ & $\mathrm{TX} / \mathrm{T}$ & 322.0 & 4.0 & 180.0 & $<1.0$ & Plastic, Cloth, Rubber, Paper, Metal \\
\hline $95-044-04$ & $<0.5$ & U & 206.4 & 4.0 & 260.0 & 2.0 & Plastic, Cloth, Rubber, Paper, Metal, Asbestos \\
\hline $95-062-03$ & $<0.5$ & $\mathrm{u}$ & 145.2 & 4.0 & 120.0 & $<1.0$ & Plastic, Paper, Weeds \\
\hline
\end{tabular}


LLW TO ATG

SHIPMENT DATE: 4-21-95

\begin{tabular}{|c|c|c|c|c|c|c|c|}
\hline PIN\# & $6 \mathrm{pt}$ & Origin & Weight & Volume & \multicolumn{2}{|c|}{ ASSAY } & \multirow[t]{2}{*}{ CONTENTS } \\
\hline & $(\mathrm{mr} / \mathrm{hr})$ & & $(\mathrm{Kg})$ & $\left(m^{3}\right)$ & Cs-137 & Co-60 & \\
\hline \multicolumn{8}{|c|}{$(\mathrm{pCi} / \mathrm{g})$} \\
\hline 94-273-01 & $<0.5$ & $209 E$ & 349.3 & 4.0 & 11.0 & 5.1 & Plastic, Cloth, Rubber, Paper, Wood, Metal \\
\hline $94-315-05$ & $<0.5$ & A & 299.4 & 4.0 & 300.0 & 0.95 & Plastic, Cloth, Rubber, Paper, Metal \\
\hline \multirow[t]{2}{*}{$95-059-02$} & $<0.5$ & AY & 233.6 & 4.0 & 1300.0 & 1.8 & Plastic, Cloth, Rubber, Paper, Wood, Metal \\
\hline & & AY & & Eu-154 & 78.45 & Cs-134 & 1.85 \\
\hline \multirow[t]{2}{*}{$95-072-05$} & $<0.5$ & AY & 367.4 & 4.0 & 17.0 & 0.8 & Plastic, Cloth, Rubber, Paper, Wood, Metal \\
\hline & & AY & & & Cs-134 1.8 & U-2 & 353.2 \\
\hline \multirow[t]{2}{*}{$95-038-06$} & $<0.5$ & BXVBY & 197.3 & 4.0 & 2900.0 & 1.8 & Plastic, Cloth, Rubber, Paper, Metal, Asbestos \\
\hline & & BXVBY & & & Eu-15 & 46.1 & \\
\hline \multirow[t]{2}{*}{$95-062-07$} & $<0.5$ & BXJBY & 172.4 & 4.0 & 740.0 & 5.45 & Plastic, Cloth, Rubber, Paper, Metal \\
\hline & & BXJBY & & & Eu-15 & 343.8 & \\
\hline \multirow[t]{2}{*}{$95-062-05$} & $<0.5$ & C & 197.3 & 4.0 & 340.0 & 2.2 & Plastic, Cloth, Rubber, Paper, Metal \\
\hline & & C & & & Eu-15 & 44.2 & \\
\hline \multirow[t]{2}{*}{$95-068-01$} & $<0.5$ & $\mathrm{C}$ & 290.3 & 4.0 & 1100.0 & 3.8 & Plastic, Cloth, Rubber, Paper, Metal \\
\hline & & C & & & Eu-15 & 44.7 & \\
\hline $95-060-02$ & $<0.5$ & sX & 174.6 & 4.0 & 160.0 & $<1$ & Plastic, Cloth, Rubber, Paper, Wood, Metal \\
\hline $95-062-04$ & $<0.5$ & $\mathbf{U}$ & 244.9 & 4.0 & 2300.0 & 1.5 & Plastic, Cloth, Rubber, Paper, Wood, Metal \\
\hline
\end{tabular}


LLW TO ATG

SHIPMENT DATE: 5-4-95

\begin{tabular}{|c|c|c|c|c|c|c|c|}
\hline PIN \# & $6 \mathrm{pt}$ & Origin & Weight & Volume & \multicolumn{2}{|c|}{ ASSAY } & CONTENTS \\
\hline & \multicolumn{2}{|l|}{$(\mathrm{mr} / \mathrm{hr})$} & $(\mathrm{Kg})$ & $\left(\mathrm{m}^{3}\right)$ & Cs-137 & Co-60 & \\
\hline \multicolumn{8}{|c|}{ (pCi/g) } \\
\hline 95-072-02 & $<0.5$ & $\mathrm{BX} / \mathrm{BY}$ & 220.0 & 4.0 & 480.0 & 1.80 & Plastic, Cloth, Rubber, Paper, Metal \\
\hline $95-074-05$ & $<0.5$ & C & 326.6 & 4.0 & 720.0 & 2.7 & Plastic, Cloth, Rubber, Paper, Wood, Metal \\
\hline $95-080-04$ & $<0.5$ & $\mathrm{C}$ & 251.7 & 4.0 & 280.0 & 1.8 & Plastic, Cloth, Rubber, Paper, Metal \\
\hline \multicolumn{8}{|c|}{ Eu-154 5.5} \\
\hline $95-074-01$ & $<0.5$ & $\mathbf{U}$ & 265.4 & 4.0 & 2600.0 & $<1$ & Plastic, Cloth, Rubber, Paper, Wood, Metal \\
\hline & & $\mathbf{U}$ & & & Eu-1 & 7.9 & \\
\hline
\end{tabular}


LLW TO ATG

SHIPMENT DATE: 5-12-95

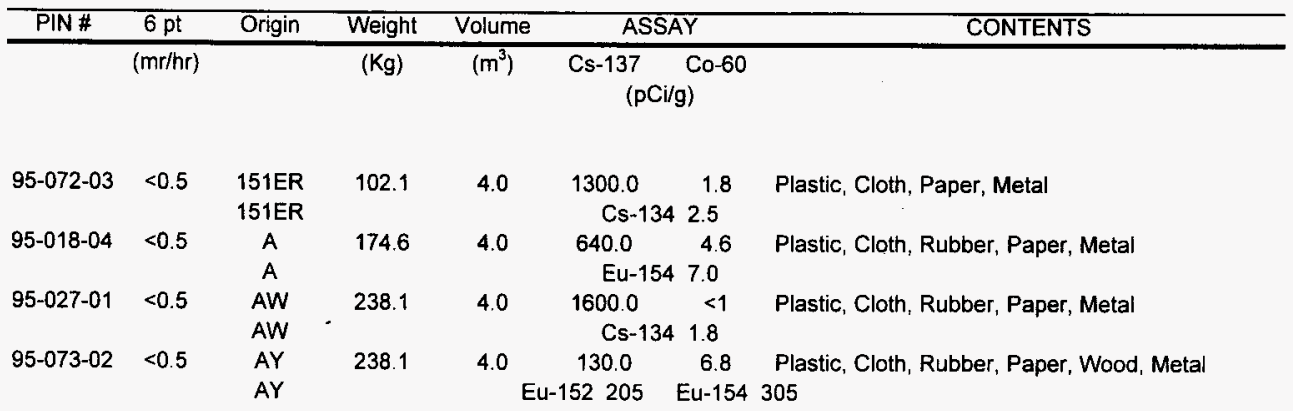


LLW TO ATG

SHIPMENT DATE: 5-26-95

\begin{tabular}{|c|c|c|c|c|c|c|c|}
\hline PIN\# & $6 \mathrm{pt}$ & Origin & Weight & Volume & \multicolumn{2}{|c|}{ ASSAY } & CONTENTS \\
\hline & $(\mathrm{mr} / \mathrm{hr})$ & & $(\mathrm{Kg})$ & $\left(m^{3}\right)$ & \multicolumn{2}{|c|}{$(\mathrm{pCi} / \mathrm{g})$} & \\
\hline $95-090-04$ & $<0.5$ & $\begin{array}{l}244 A \\
244 A\end{array}$ & 147.4 & 4.0 & $\begin{array}{l}260.0 \\
\text { Eu-1 }\end{array}$ & $\begin{array}{l}0.44 \\
1.8\end{array}$ & Plastic, Cloth, Rubber, Paper, Metal \\
\hline $95-088-04$ & $<0.5$ & A & 174.6 & 4.0 & 5300.0 & 2.2 & Plastic, Cloth, Rubber, Paper, Metal \\
\hline $95-062-06$ & $<0.5$ & AN & 201.9 & 4.0 & 340.0 & 5.35 & Plastic, Cloth, Rubber, Paper, Metal \\
\hline $95-088-03$ & $<0.5$ & BX/BY & 242.7 & 4.0 & 720.0 & $<1$ & Plastic, Cloth, Rubber, Paper, Metal, Asbestos \\
\hline $95-086-06$ & $<0.5$ & C & 392.4 & 4.0 & 830.0 & 0.59 & Plastic, Cloth, Rubber, Paper, Wood, Metal \\
\hline 95-039-01 & $<0.5$ & $\mathrm{~S}$ & 88.5 & 4.0 & 410.0 & $<1$ & Plastic, Cloth, Rubber, Paper, Wood, Metal \\
\hline $95-072-04$ & $<0.5$ & s/SX & 274.4 & 4.0 & 280.0 & $<1.0$ & Plastic, Cloth, Rubber, Paper, Metal \\
\hline $95-040-04$ & $<0.5$ & $\begin{array}{l}S Y \\
S Y\end{array}$ & 197.3 & 4.0 & 990.0 & 2.1 & Plastic, Cloth, Rubber, Paper, Wood, Metal, Concrete \\
\hline $95-087-07$ & $<0.5$ & $\mathbf{U}$ & 333.0 & 4.0 & 400.0 & $<1$ & Plastic, Cloth, Rubber, Paper, Wood, Metal, Asbestos \\
\hline
\end{tabular}


LLW TO ATG

SHIPMENT DATE: 6-29-95

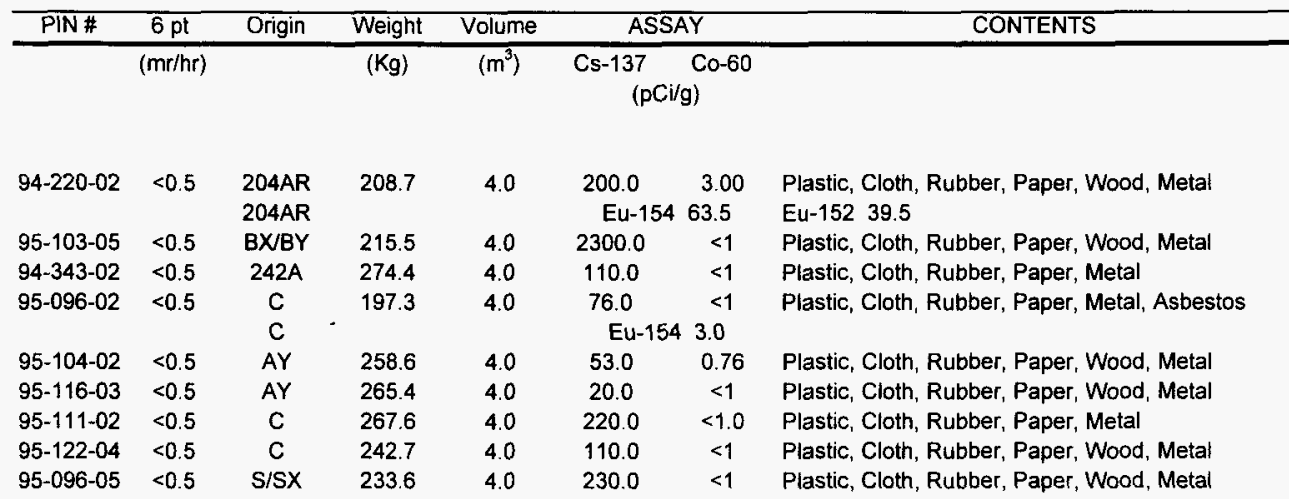


LLW TO ATG

SHIPMENT DATE: 7-13-95

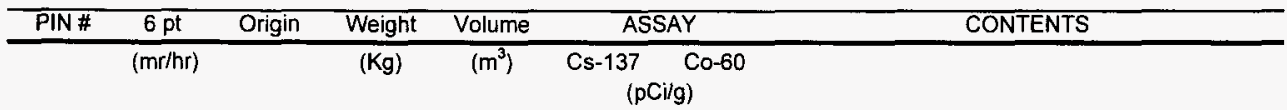

\begin{tabular}{|c|c|c|c|c|c|c|c|}
\hline \multirow[t]{2}{*}{$95-093-02$} & 0.4 & AW & 220.0 & 4.0 & 27.0 & 1.40 & Plastic, Cloth, Rubber, Paper, Wood, Metal \\
\hline & \multicolumn{6}{|c|}{ Eu-154 1.7} & \\
\hline $95-040-05$ & 0.4 & $T$ & 301.6 & 4.0 & 400.0 & $<1$ & Plastic, Cloth, Rubber, Paper, Wood, Metal \\
\hline $95-135-03$ & 0.4 & $B X / B Y$ & 260.8 & 4.0 & 580.0 & 0.97 & Plastic, Cloth, Rubber, Paper, Wood, Metal \\
\hline $95-135-02$ & 0.4 & C & 197.3 & 4.0 & 70.0 & $<1$ & Plastic, Cloth, Rubber, Paper, Wood, Metal \\
\hline $95-065-02$ & 0.4 & $\mathbf{B}$ & 365.1 & 4.0 & 320.0 & $<1$ & Plastic, Cloth, Rubber, Paper, Wood, Metal \\
\hline $95-139-05$ & 0.4 & S/SX & 161.0 & 4.0 & 320.0 & $<1$ & Plastic, Cloth, Rubber, Paper, Wood, Metal \\
\hline $95-060-01$ & 0.4 & TX/TY & 260.8 & 4.0 & 810.0 & 1.5 & Plastic, Cloth, Rubber, Paper, Wood, Metal, Asbestos \\
\hline $95-130-02$ & 0.4 & C & 229.1 & 4.0 & 1100.0 & 4.0 & Plastic, Cloth, Rubber, Paper, Metal \\
\hline $95-096-06$ & 0.4 & SY & 165.1 & 4.0 & 180.0 & $<1$ & Plastic, Cloth, Rubber, Paper, Metal, Asbestos \\
\hline $95-135-04$ & 0.4 & S/SX & 269.9 & 4.0 & 50.0 & $<1$ & Plastic, Cloth, Rubber, Paper, Wood, Metal \\
\hline
\end{tabular}


LLW TO ATG

SHIPMENT DATE: 8-1-95

\begin{tabular}{|c|c|c|c|c|c|}
\hline PIN\# & $6 \mathrm{pt}$ & Origin & Weight & Volume & ASSAY \\
\hline & $(\mathrm{mr} / \mathrm{hr})$ & & $(\mathrm{Kg})$ & $\left(\mathrm{m}^{3}\right)$ & Cs-137 Co-60 \\
\hline
\end{tabular}

\begin{tabular}{cccccccl}
$95-132-49$ & 0.4 & AY & 308.9 & 4.0 & 4.2 & 1.40 & Plastic, Cloth, Rubber, Paper, Wood, Metal, Asbestos \\
$95-103-01$ & 0.4 & 244 A & 285.8 & 4.0 & 15.0 & $<1$ & Plastic, Cloth, Rubber, Paper, Dirt, Metal \\
$95-146-05$ & 0.4 & B & 238.1 & 4.0 & 300.0 & $<1$ & Plastic, Cloth, Rubber, Paper, Metal, Asbestos \\
& & B & & \multicolumn{4}{c}{ Eu-154 2.2} \\
$95-146-02$ & 1.3 & BX/BY & 280.8 & 4.0 & $620.0 \quad<1$ & Plastic, Cloth, Rubber, Paper, Wood, Metal
\end{tabular}


LLW TO ATG

SHIPMENT DATE: $10-3-95$

\begin{tabular}{|c|c|c|c|c|c|c|}
\hline PIN \# & $6 \mathrm{pt}$ & Origin & Weight & Volume & ASSAY & CONTENTS \\
\hline & $(\mathrm{mr} / \mathrm{hr})$ & & $(\mathrm{Kg})$ & $\left(m^{3}\right)$ & Cs-137 Co-60 & \\
\hline
\end{tabular}

$\begin{array}{llcllccl}95-125-03 & 0.4 & \text { SY } & 301.6 & 4.0 & 190.0 & 0.68 & \text { Plastic, Cloth, Rubber, Paper, Wood, Metal, Asbestos } \\ 95-139-06 & <0.5 & \text { SX } & 288.0 & 4.0 & 56.0 & <1 & \text { Plastic, Cloth, Rubber, Paper, Wood, Metal } \\ 95-116-01 & 0.4 & \text { AN } & 256.3 & 4.0 & 1900.0 & 2.10 & \text { Plastic, Cloth, Rubber, Paper, Dirt, Metal, Asbestos } \\ 95-165-01 & 0.4 & \text { B } & 301.6 & 4.0 & 320.0 & <1 & \text { Plastic, Cloth, Rubber, Paper, Wood, Metal, Asbestos } \\ 95-146-04 & 0.4 & \text { T } & 260.8 & 4.0 & 260.0 & 1.00 & \text { Plastic, Cloth, Rubber, Paper, Wood, Metal, Asbestos } \\ 95-144-04 & 0.4 & \text { TXTY } & 235.9 & 4.0 & 1500.0 & 1.2 & \text { Plastic, Cloth, Rubber, Paper, Wood, Metal, Asbestos } \\ 95-172-02 & 0.4 & \text { SY } & 183.7 & 4.0 & 340.0 & 0.7 & \text { Plastic, Cloth, Rubber, Paper, Wood, Metal, Foam } \\ 95-177-01 & 0.4 & \text { B } & 306.2 & 4.0 & 350.0 & 3.1 & \text { Plastic, Cloth, Rubber, Paper, Wood, Metal, Asbestos } \\ 95-065-03 & 0.4 & \text { A } & 358.3 & 4.0 & 120.0 & 0.9 & \text { Plastic, Cloth, Rubber, Paper, Metal, Asbestos } \\ 95-174-02 & 0.4 & \text { AN } & 288.0 & 4.0 & 470.0 & <1 & \text { Plastic, Cloth, Rubber, Paper, Wood, Metal, Asbestos }\end{array}$


LLW TO ATG

SHIPMENT DATE: 10-24-95

\begin{tabular}{|c|c|c|c|c|c|c|}
\hline PIN\# & $6 \mathrm{pt}$ & Origin & Weight & Volume & ASSAY & CONTENTS \\
\hline & $(\mathrm{mr} / \mathrm{hr})$ & & $(\mathrm{Kg})$ & $\left(\mathrm{m}^{3}\right)$ & Cs-137 Co-60 & \\
\hline
\end{tabular}

\begin{tabular}{|c|c|c|c|c|c|c|c|}
\hline $95-074-06$ & 0.4 & $\begin{array}{l}209 E \\
209 E\end{array}$ & 287.1 & 4.0 & $\begin{array}{l}460.0 \\
\text { Eu-15 }\end{array}$ & $\begin{array}{l}0.66 \\
7.5\end{array}$ & Plastic, Cloth, Rubber, Paper, Wood, Metal, Foam \\
\hline $95-146-03$ & 0.4 & C & 337.9 & 4.0 & 59.0 & 0.8 & Plastic, Cloth, Rubber, Paper, Wood, Metal, Insulation \\
\hline $95-205-04$ & 0.4 & $\mathrm{BX} / \mathrm{BY}$ & 238.1 & 4.0 & 2800.0 & 2.40 & Plastic, Cloth, Rubber, Paper, Metal, Vegetation \\
\hline $95-160-02$ & 0.4 & AY & 237.7 & 4.0 & 19.0 & 3.1 & Plastic, Cloth, Rubber, Paper, Wood, Metal \\
\hline \multirow[t]{2}{*}{$95-116-02$} & 0.4 & $242 A$ & 296.2 & 4.0 & 4000.0 & 5.15 & Plastic, Cloth, Rubber, Paper, Metal, Glass \\
\hline & & $242 A$ & & & Eu-152 2.3 & Eu-154 & $2.0 \mathrm{Cs}-1347.65$ \\
\hline $95-101-01$ & 0.4 & U & 383.3 & 4.0 & 350.0 & 0.4 & Plastic, Cloth, Rubber, Paper, Wood, Metal, Asbestos \\
\hline $95-122-03$ & 0.4 & 2ETF & 324.3 & 4.0 & 820.0 & 1.4 & Plastic, Cloth, Rubber, Paper, Wood, Metal \\
\hline $95-144-03$ & 0.4 & EWTF & 201.9 & 4.0 & 490.0 & 1.0 & Plastic, Cloth, Rubber, Paper, Wood, Metal, Asbestos \\
\hline \multirow[t]{2}{*}{$95-223-03$} & 0.4 & 2ETF & 406.0 & 4.0 & 1100.0 & 3.5 & Plastic, Cloth, Rubber, Paper, Metal, Asbestos \\
\hline & & 2ETF & & & Eu-15 & 2.2 & \\
\hline
\end{tabular}


LLW TO ATG

SHIPMENT DATE: 12-21-95

\begin{tabular}{ccccccc}
\hline PIN\# & $6 \mathrm{pt}$ & Origin & Weight & Volume & ASSAY & CONTENTS \\
\hline & $(\mathrm{mr} / \mathrm{hr})$ & & $(\mathrm{Kg})$ & $\left(\mathrm{m}^{3}\right)$ & $\mathrm{Cs}-137 \underset{(\mathrm{pCi} / \mathrm{g})}{\mathrm{Co}-60}$ &
\end{tabular}

\begin{tabular}{|c|c|c|c|c|c|c|c|}
\hline & 0.6 & $\mathrm{BX}$ & 242.7 & 4.0 & 1800.0 & 1.50 & Plastic, Cloth, Rubber, Paper, Wood, Metal, Foam \\
\hline 5-181-1 & 1.0 & $\mathrm{BX}$ & 269.9 & 4.0 & 2600.0 & 1.5 & Plastic, Cloth, Rubber, Paper, Wood, Metal \\
\hline
\end{tabular}


LLW TO ATG

SHIPMENT DATE: 2-5-96

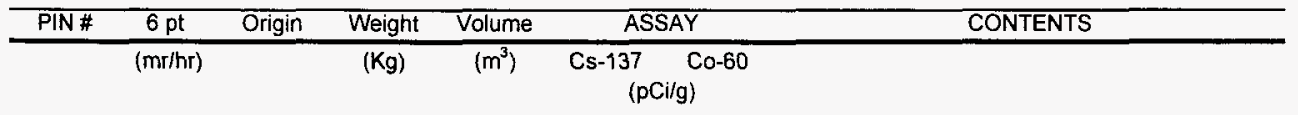

\begin{tabular}{|c|c|c|c|c|c|c|c|}
\hline $95-165-02$ & 0.4 & $B Y / 244 A$ & 179.2 & 4.0 & 620.0 & 2.30 & Plastic, Cloth, Rubber, Paper, Wood, Metal \\
\hline & & $\mathrm{BY} / 244 \mathrm{~A}$ & & & Eu-152 & 7.4 & Eu-154: 3.6 \\
\hline $95-061-02$ & 0.4 & $200 E$ & 365.1 & 4.0 & 38.0 & 0.5 & Plastic, Cloth, Rubber, Paper, Wood, Metal, Vegetation \\
\hline $95-227-01$ & 0.4 & $200 \mathrm{~W}$ & 238.1 & 4.0 & 2300.0 & 1.50 & Plastic, Cloth, Rubber, Paper, Wood, Metal, Vegetation \\
\hline $95-272-01$ & 1.5 & $200 E$ & 360.6 & 4.0 & 2300.0 & 3.0 & Plastic, Cloth, Rubber, Paper, Wood, Metal \\
\hline $95-173-05$ & 0.4 & BY/242A & 587.4 & 4.0 & 1000.0 & $<1$ & Plastic, Cloth, Soil, Fiberglass, Asbestos \\
\hline $95-283-03$ & 0.4 & $242 A$ & 369.7 & 4.0 & 19.0 & 7.5 & Plastic, Cloth, Rubber, Paper, Metal \\
\hline $95-269-01$ & 0.4 & $\begin{array}{l}200 E \\
200 E\end{array}$ & 206.4 & 4.0 & $\begin{array}{l}2800.0 \\
\text { Eu-152 }\end{array}$ & $\begin{array}{r}<1 \\
105\end{array}$ & $\begin{array}{l}\text { Plastic, Cloth, Rubber, Paper, Metal } \\
\text { Eu-154: } 165\end{array}$ \\
\hline
\end{tabular}




\section{LLW TO ATG \\ SEA-LAND CONTAINERS}

\begin{tabular}{|c|c|c|c|c|c|c|c|c|c|}
\hline \multirow{2}{*}{$\begin{array}{c}\text { Shipment } \\
\text { Date }\end{array}$} & \multirow[t]{2}{*}{ PIN\# } & \multirow{2}{*}{$\frac{6 \mathrm{pt}}{(\mathrm{mr} / \mathrm{hr})}$} & \multirow[t]{2}{*}{ Origin } & \multicolumn{2}{|c|}{ Weight Volume } & \multirow[t]{2}{*}{ Assay PIN\# } & \multicolumn{2}{|c|}{ From Assay } & \multirow[t]{3}{*}{ CONTENTS } \\
\hline & & & & $(\mathrm{Kg})$ & $\left(m^{3}\right)$ & & Cs-137 & $\mathrm{Co}-60$ & \\
\hline & & & & & & & \multicolumn{2}{|c|}{$(\mathrm{pCi} / \mathrm{g})$} & \\
\hline \multirow[t]{2}{*}{$11 / 14 / 95$} & $95-223-04$ & 0.4 & 2ETF & 3435.0 & 33.1 & $95-223-3$ & 1100.0 & 3.50 & Plastic, Cloth, Rubber, Paper, Wood, Metal, Vegetation, Asbestos \\
\hline & & & 2ETF & & & & \multicolumn{2}{|c|}{ Eu-154: 2.2} & \\
\hline 1/16/96 & $95-223-02$ & 0.4 & $200 \mathrm{~W}$ & 2633.0 & 33.1 & $95-227-01$ & 2300.0 & 1.5 & Plastic, Cloth, Rubber, Paper, Wood, Metal, Vegetation, Asbestos \\
\hline \multirow[t]{2}{*}{$1 / 18 / 96$} & $95-265-02$ & 0.4 & $200 E$ & 2442.0 & 33.1 & $95-269-01$ & 2800.0 & 6.90 & Plastic, Cloth, Rubber, Paper, Wood, Metal, Vegetation, Asbestos \\
\hline & & & $200 \mathrm{E}$ & & & & \multicolumn{2}{|c|}{ Eu-152: 105} & Eu-154: 165 \\
\hline 2/20/96 & $95-278-01$ & 0.4 & $200 \mathrm{~W}$ & 2196.3 & 33.1 & & 2100.0 & 1.7 & Plastic, Cloth, Rubber, Paper, Wood, Metal, Vegetation, Asbestos \\
\hline \multirow[t]{2}{*}{$3 / 1 / 96$} & $95-304-4$ & 0.4 & $200 E$ & 2980.0 & 33.1 & & 450.0 & 1.74 & Plastic, Cloth, Rubber, Paper, Wood, Metal, Vegetation, Asbestos \\
\hline & & & & & & & \multicolumn{2}{|c|}{ Eu-154: 4.6} & \\
\hline \multirow[t]{2}{*}{$3 / 26 / 96$} & 95-331-03 & 0.4 & $200 \mathrm{E}$ & 2734.7 & 33.1 & & 2.2 & 3.2 & Plastic, Cloth, Rubber, Paper, Wood, Metal, Vegetation, Asbestos \\
\hline & & & $200 E$ & & & & \multicolumn{2}{|c|}{ Eu-152: 8.0} & Eu-154: 4.60 \\
\hline $4 / 11 / 96$ & $95-331-01$ & 0.4 & $200 \mathrm{~W}$ & 3431.0 & 33.1 & & 25.0 & 20.0 & Plastic, Cloth, Rubber, Paper, Wood, Metal, Concrete, Asbestos \\
\hline \multirow[t]{2}{*}{$5 / 2 / 96$} & $95-356-01$ & 0.4 & $200 E$ & 3102.6 & 33.1 & & 210.0 & 6.0 & Plastic, Cloth, Rubber, Paper, Wood, Metal, Vegetation, Asbestos \\
\hline & & & & & & & \multicolumn{2}{|c|}{ Eu-154: 4.1} & \\
\hline \multirow[t]{2}{*}{$5 / 21 / 96$} & $96-101-01$ & $<.5$ & 200EN & 5040.0 & 33.1 & & 648.0 & 4.3 & Plastic, Cloth, Rubber, Paper, Wood, Metal, Asbestos \\
\hline & & & & & & & Eu-154 & 1.25 & Plastic, Cloth, Rubber, Paper, Wood, Metal, Asbestos \\
\hline \multirow[t]{2}{*}{$5 / 24 / 96$} & $96-086-02$ & $<.5$ & $200 \mathrm{~W}$ & 2358.7 & 33.1 & & 648.0 & 4.3 & Plastic, Cloth, Rubber, Paper, Wood, Metal, Asbestos \\
\hline & & & & & & & \multicolumn{2}{|l|}{ Eu-154: } & \\
\hline
\end{tabular}


WHC-SD-WM-ER-435, Rev. 1

Page D- $i$

\section{ATTACHMENT D}




\section{ATG ASSAY COMPARISONS}

ATG Return Date: 3-13-95

Shipment \#: 1

Compaction Ratio: 4.8:1

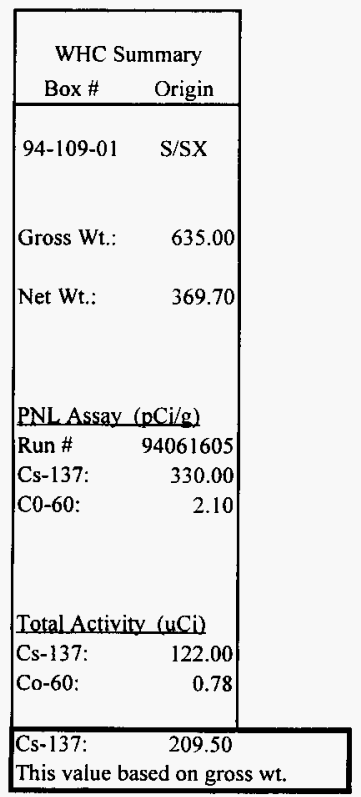

\begin{tabular}{|c|c|c|c|c|}
\hline \multicolumn{5}{|c|}{ ATG Summary } \\
\hline ATG Drum \# & PNL Assay \# & Net Wt. & $\mathrm{Cs}-137^{(1)}$ & $\mathrm{C} 0-60^{(1)}$ \\
\hline & & $(\mathrm{Kg})$ & $\mathrm{pCi} / \mathrm{g}$ & $\mathrm{pCi} / \mathrm{g}$ \\
\hline 49 & 95012309 & 44.90 & 410.00 & 5.10 \\
\hline 50 & 95012313 & 63.50 & 16.00 & 5.20 \\
\hline 51 & 95012308 & 70.80 & 66.00 & \\
\hline 52 & 95012306 & 68.50 & 54.00 & \\
\hline 53 & 95012310 & 66.70 & & \\
\hline 54 & 95012304 & 54.00 & & 4.10 \\
\hline 55 & 95012302 & 49.90 & & \\
\hline 56 & 95012305 & 45.40 & 31.00 & \\
\hline 57 & 95012303 & 39.50 & & \\
\hline 58 & 95012311 & 46.30 & & \\
\hline 59 & 95012307 & 29.00 & & \\
\hline TOTALS: & & 578.50 & 577.00 & 14.40 \\
\hline \multicolumn{5}{|c|}{ TotalActivity (uCi) } \\
\hline Cs-137: & 333.79 & & & \\
\hline Co-60: & 8.33 & & & \\
\hline
\end{tabular}

(1) Blank cell indicates a less than value.

For this compaction shipment the shipping box was also dismantled and compacted. 


\section{ATGASSAY COMPARISONS}

ATG Retum Date: $3-24-95$

Shipment \#: 4

Compaction Ratio: 7.42:1

\begin{tabular}{|c|c|}
\hline \multicolumn{2}{|c|}{ WHC Summary } \\
\hline Box \# & Origin \\
\hline $94-249-02$ & S/SX \\
\hline Gross Wt:: & 644.00 \\
\hline Net Wt.: & 337.90 \\
\hline \multicolumn{2}{|c|}{ PNLAssay $(\mathrm{nCi} / \mathrm{g})$} \\
\hline Run \# & 95012605 \\
\hline Cs-137: & 1700.00 \\
\hline $\mathrm{CO}-60:$ & 3.10 \\
\hline \multicolumn{2}{|c|}{ Total Activity (uCi) } \\
\hline Cs-137: & 574.00 \\
\hline Co-60: & 1.05 \\
\hline
\end{tabular}

\begin{tabular}{|c|c|c|c|c|}
\hline \multicolumn{5}{|c|}{ ATG Summary } \\
\hline ATG Drum \# & PNL Assay \# & Net Wt. & Cs-137 ${ }^{(1)}$ & $\mathrm{Co}-60^{(1)}$ \\
\hline & & $(\mathrm{Kg})$ & $\mathrm{pCi} / \mathrm{g}$ & $\mathrm{pCi} / \mathrm{g}$ \\
\hline I & 95020809 & 65.80 & 740.00 & \\
\hline 2 & 95020810 & 80.30 & 440.00 & 7.30 \\
\hline 3 & 95020815 & 81.20 & 22.00 & \\
\hline 4 & 95020812 & 57.20 & 4.00 & 1.10 \\
\hline 5 & 95020813 & 56.30 & 9.90 & 1.10 \\
\hline 6 & 95020814 & 73.90 & 490.00 & \\
\hline TOTALS: & & 414.70 & 1705.90 & 9.50 \\
\hline \multicolumn{5}{|c|}{ Total Activity (uCi) } \\
\hline Cs-137: & 707.44 & & & \\
\hline Co-60: & 3.94 & & & \\
\hline
\end{tabular}

(1) Blank cell indicates a less than value.

The Net wt. of the compacted waste from this container is $76.8 \mathrm{~kg}$ more than the Net wt. on the shipping papers. The Net wt. of the compacted waste for container $94-159-03$ is $77.9 \mathrm{~kg}$ less than the Net wt. on the shipping papers. Both boxes were from S/SX farm. It appears that waste from one container was compacted with waste from the other container. 


\section{ATG ASSAY COMPARISONS}

ATG Return Date: 3-24-95

Shipment \#: 4

Compaction Ratio: $9.16: 1$

\begin{tabular}{|c|c|}
\hline \multicolumn{2}{|c|}{ WHC Summary } \\
\hline Box \# & Origin \\
\hline $94-159-03$ & S/SX \\
\hline Gross Wt:: & 658.00 \\
\hline Net Wt.: & 351.50 \\
\hline \multicolumn{2}{|c|}{ PNL Assay $(p \mathrm{Ci} / \mathrm{g})$} \\
\hline Run \# & 95012505 \\
\hline Cs-137: & 49.00 \\
\hline $\mathrm{C} 0.60:$ & 0.88 \\
\hline \multicolumn{2}{|c|}{ Total Activity (uCi) } \\
\hline Cs-137: & 17.20 \\
\hline Co-60: & 0.31 \\
\hline
\end{tabular}

\begin{tabular}{|c|c|c|c|c|}
\hline \multicolumn{5}{|c|}{ ATG Summary } \\
\hline ATG Drum \# & PNL Assay \# & Net Wt. & Cs-137 (1) & $\mathrm{Co}-60^{(1)}$ \\
\hline & & $(\mathrm{Kg})$ & $\mathrm{pCi} / \mathrm{g}$ & $\mathrm{pCi} / \mathrm{g}$ \\
\hline 7 & 95020804 & 46.30 & & 1.80 \\
\hline 8 & 95020803 & 61.70 & & \\
\hline 9 & 95020805 & 55.80 & & 3.20 \\
\hline 10 & 95020806 & 56.20 & & 2.70 \\
\hline 11 & 95020808 & 21.80 & & \\
\hline 12 & 95020807 & 31.80 & & 2.80 \\
\hline TOTALS: & & 273.60 & 0.00 & 10.50 \\
\hline \multicolumn{5}{|c|}{ Total Activity (uCi) } \\
\hline Cs-137: & 0.00 & & & \\
\hline & 2.87 & & & \\
\hline
\end{tabular}

(1) Blank cell indicates a less than value. 


\section{ATG ASSAY COMPARISONS}

ATG Return Date: $3-31-95$

Shipment \#: 5

Compaction Ratio: 8.11:1

\begin{tabular}{|lc|}
\hline \multicolumn{2}{|c|}{ WHC Summary } \\
Box \# & Origin \\
\hline 94-270-09 & BX/BY \\
& \\
Gross Wt.: & 667.00 \\
Net Wt.: & 360.60 \\
& \\
& \\
PNLAssay & (pCi/g) \\
Run \# & 95012405 \\
Cs-137: & 910.00 \\
C0-60: & 2.30 \\
& \\
& \\
Total Activity & (uCi) \\
Cs-137: & 328.00 \\
Co-60: & 0.83 \\
& \\
\hline &
\end{tabular}

\begin{tabular}{|c|c|c|c|c|}
\hline \multicolumn{5}{|c|}{ ATG Summary } \\
\hline ATG Drum \# & PNL Assay \# & Net Wt. & Cs- $137^{(1)}$ & $\mathrm{Co}-60^{(1)}$ \\
\hline & & $(\mathrm{Kg})$ & $\mathrm{pCi} / \mathrm{g}$ & $\mathrm{pCi} / \mathrm{g}$ \\
\hline 32 & 95021602 & 43.10 & 410.00 & \\
\hline 33 & 95021603 & 44.50 & 81.00 & \\
\hline 34 & 95021604 & 54.90 & 110.00 & \\
\hline 35 & 95021605 & 47.60 & 200.00 & \\
\hline 36 & 95021606 & 43.10 & 36.00 & 1.40 \\
\hline 37 & 95021607 & 151.50 & 170.00 & \\
\hline TOTALS: & & 384.70 & 1007.00 & I. 40 \\
\hline \multicolumn{5}{|c|}{ Total Activity (uCi) } \\
\hline Cs-137: & 387.39 & & & \\
\hline Co-60: & 0.54 & & & \\
\hline
\end{tabular}

(1) Blank cell indicates a less than value. 


\section{ATG ASSAY COMPARISONS}

ATG Return Date: 4-05-95

Shipment \#: 6

Compaction Ratio: 8.76:1

\begin{tabular}{|c|c|}
\hline \multicolumn{2}{|c|}{ WHC Summary } \\
\hline Box \# & Origin \\
\hline $94-252-01$ & $\mathrm{BX} / \mathrm{BY}$ \\
\hline Gross Wt.: & 567.00 \\
\hline Net Wt.: & 260.80 \\
\hline \multicolumn{2}{|c|}{ PNL Assay (pCi/g) } \\
\hline Run \# & 9501307 \\
\hline Cs.137: & 860.00 \\
\hline C0-60: & 1.50 \\
\hline \multicolumn{2}{|c|}{ Total Activity (uCi) } \\
\hline Cs-137: & 224.29 \\
\hline Co-60: & 0.39 \\
\hline
\end{tabular}

\begin{tabular}{|c|c|c|c|c|}
\hline \multicolumn{5}{|c|}{ ATG Summary } \\
\hline ATG Drum \# & PNL Assay \# & Net Wt. & $\mathrm{Cs}-137^{(1)}$ & $\mathrm{Co}-60^{(1)}$ \\
\hline & & $(\mathrm{Kg})$ & $\mathrm{pCi} / \mathrm{g}$ & $\mathrm{pCi} / \mathrm{g}$ \\
\hline 1 & 95021609 & 51.70 & 14.00 & \\
\hline 2 & 95021610 & 66.20 & 52.00 & 3.10 \\
\hline 3 & 95021611 & 70.30 & 290.00 & \\
\hline 4 & 95021612 & 80.70 & 24.00 & \\
\hline 5 & 95021613 & 52.20 & 440.00 & \\
\hline TOTALS: & & 321.10 & 820.00 & 3.10 \\
\hline \multicolumn{5}{|c|}{ Total Activity (uCi) } \\
\hline Cs-137: & 263.30 & & & \\
\hline Co- $60:$ & 1.00 & & & \\
\hline
\end{tabular}

(1) Blank cell indicates a less than value. 


\section{ATG ASSAY COMPARISONS}

ATG Return Date: 4-13-95

Shipment \#: 7

Compaction Ratio: 6.91:1

\begin{tabular}{|c|c|}
\hline \multicolumn{2}{|c|}{ WHC Summary } \\
\hline Box \# & Origin \\
\hline $94-213-02$ & $242-A$ \\
\hline Gross Wt: & 744.00 \\
\hline Net Wt:: & 437.70 \\
\hline \multicolumn{2}{|c|}{ PNL Assay (pCig) } \\
\hline Run \# & 95020805 \\
\hline Cs-137: & 240.00 \\
\hline$C 0-60:$ & 1.50 \\
\hline \multicolumn{2}{|c|}{ Total Activity (uCi) } \\
\hline Cs-137: & 105.00 \\
\hline $\mathrm{Co}-60:$ & 0.66 \\
\hline
\end{tabular}

\begin{tabular}{|c|c|c|c|c|}
\hline \multicolumn{5}{|c|}{ ATG Summary } \\
\hline ATG Drum \# & PNL Assay \# & Net Wt. & $\mathrm{Cs}-137^{(1)}$ & $\mathrm{Co}-60^{(1)}$ \\
\hline & & $(\mathrm{Kg})$ & $\mathrm{pCi} / \mathrm{g}$ & $\mathrm{pCi} / \mathrm{g}$ \\
\hline 33 & 95022402 & 97.50 & 11.00 & 1.90 \\
\hline 34 & 95022403 & 87.10 & 14.00 & \\
\hline 35 & 95022404 & 99.30 & 150.00 & 1.80 \\
\hline 36 & 95022405 & 59.00 & 170.00 & 3.40 \\
\hline 37 & 95022406 & 77.60 & & \\
\hline 38 & 95022407 & 53.50 & 19.00 & 3.10 \\
\hline TOTALS: & & 474.00 & 364.00 & 10.20 \\
\hline \multicolumn{5}{|c|}{ Total Activity (uCi) } \\
\hline Cs-137: & 172.54 & & & \\
\hline Co-60: & 4.83 & & & \\
\hline
\end{tabular}

(1) Blank cell indicates a less than value. 


\section{ATG ASSAY COMPARISONS}

ATG Return Date: 4-17-95

Shipment \#: 8

Compaction Ratio: 10.16:1

\begin{tabular}{|lc|}
\hline \multicolumn{2}{|c|}{ WHC Summary } \\
Box \# & Origin \\
\hline 94-343-01 & AY \\
& \\
Gross Wt.: & 571.00 \\
Net Wt.: & 265.40 \\
& \\
& \\
PNLAssay & $(\mathrm{pCi} / \mathrm{g})$ \\
Run \# & 95021405 \\
Cs-137: & 170.00 \\
C0-60: & 0.08 \\
& \\
& \\
Total Activity & $(\mathrm{uCi})$ \\
Cs-137: & 45.10 \\
Co-60: & 0.02 \\
\hline & \\
\hline
\end{tabular}

\begin{tabular}{|c|c|c|c|c|}
\hline \multicolumn{5}{|c|}{ ATG Summary } \\
\hline ATG Drum \# & PNL Assay \# & Net Wt. & $\mathrm{Cs}-137^{(1)}$ & $\mathrm{C} 0-60^{(1)}$ \\
\hline & & $(\mathrm{Kg})$ & $\mathrm{pCi} / \mathrm{g}$ & $\mathrm{pCi} / \mathrm{g}$ \\
\hline 17 & 95030703 & 64.40 & 9.90 & 2.30 \\
\hline 18 & 95030705 & 103.90 & 95.00 & \\
\hline 19 & 95030702 & 52.50 & 7.50 & 1.30 \\
\hline 20 & 95030704 & 59.40 & 14.00 & 1.40 \\
\hline TOTALS: & & 280.20 & 126.40 & 5.00 \\
\hline Total Activity & (uCi) & & & \\
\hline Cs-137: & 35.42 & & & \\
\hline $\mathrm{Co}-60:$ & 1.40 & & & \\
\hline
\end{tabular}

(1) Blank cell indicates a less than value. 


\section{ATG ASSAY COMPARISONS}

ATG Return Date: 5-15-95

Shipment \#: 9

Compaction Ratio: 7.05:1

\begin{tabular}{|lc|}
\hline \multicolumn{2}{|c|}{ WHC Summary } \\
Box \# & Origin \\
\hline 95-024-02 & C \\
& \\
Gross Wt.: & 631.00 \\
Net Wt.: & 324.30 \\
& \\
& \\
PNL Assay & (pCi $/ \mathrm{g})$ \\
Run \# & 95022707 \\
Cs-137: & 1500.00 \\
C0-60: & 6.00 \\
& \\
& \\
TotalActivity & $(u \mathrm{Ci})$ \\
Cs-137: & 486.00 \\
Co-60: & 1.95 \\
& \\
\hline
\end{tabular}

\begin{tabular}{|c|c|c|c|c|}
\hline \multicolumn{5}{|c|}{ ATG Summary } \\
\hline ATG Drum \# & PNL Assay \# & Net Wt. & Cs-137 ${ }^{(1)}$ & $\mathrm{Co}-60^{(1)}$ \\
\hline & & $(\mathrm{Kg})$ & $\mathrm{pCi} / \mathrm{g}$ & $\mathrm{pCi} / \mathrm{g}$ \\
\hline 31 & 95032805 & 119.80 & 110.00 & 15.00 \\
\hline 32 & 95032802 & 104.80 & 120.00 & 0.94 \\
\hline 33 & 95032804 & 54.90 & 1300.00 & 1.30 \\
\hline 34 & 95032803 & 86.60 & 73.00 & 0.89 \\
\hline TOTALS: & & 366.10 & 1603.00 & 18.13 \\
\hline Total Activity & (uCi) & & & \\
\hline Cs-137: & 586.86 & & & \\
\hline Co-60: & 6.64 & & & \\
\hline
\end{tabular}

(1) Blank cell indicates a less than value. 


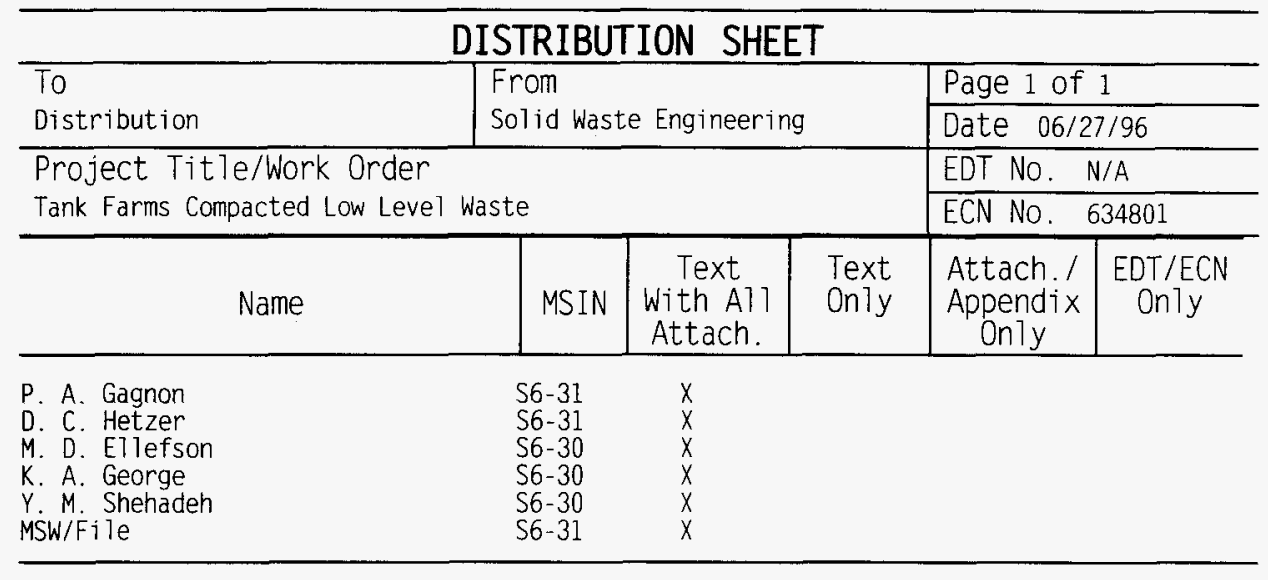

Central Files

A3-88 $X$

